Supporting Information for:

\title{
Bulky Alkylphosphines with Neopentyl Substituents as Ligands in the Amination of Aryl Bromides and Chlorides
}

Lensey L. Hill, ${ }^{a}$ Lucas R. Moore, ${ }^{a}$ Rongcai Huang, ${ }^{a}$ Raluca Craciun, ${ }^{a}$ Andrew Vincent, ${ }^{a}$ David A. Dixon, $*^{a}$ Joe Chou, ${ }^{b}$ Christopher J. Woltermann, ${ }^{b}$ and Kevin H. Shaughnessy $*^{* a}$

${ }^{a}$ Department of Chemistry, The University of Alabama, Box 870336, Tuscaloosa, AL 354870336.

${ }^{\mathrm{b}}$ FMC Corporation, Lithium Division, Hwy 161, Box 795, Bessemer City, NC 28016-0795.

\section{Table of Contents}

Experimental Details and Characterization Data ........................................................................

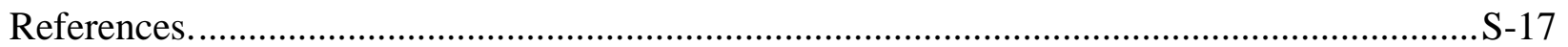

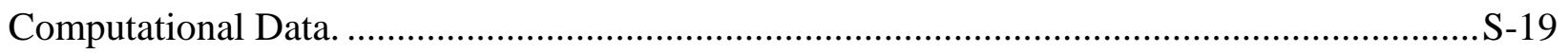

${ }^{1} \mathrm{H}$ and ${ }^{13} \mathrm{C}$ NMR Spectra of Amine Products ......................................................................... 


\section{Experimental Details and Characterization Data}

General Procedures. TTBP, DTBNpP, TBDNpP, and TNpP were obtained from FMC, Lithium Division as $10-15 \%(\mathrm{w} / \mathrm{w})$ solutions in toluene. These were stored and dispensed in a nitrogen filled glove box. DTBNpP·HBF 4 was also obtained from FMC, Lithium Division. Palladium sources, aryl halides, and amines were obtained from commercial sources and were used as received. Toluene and xylenes were distilled from molten sodium under nitrogen. All coupling reactions were carried out under nitrogen in septum-sealed reaction vessels.

\section{Typical procedure for the coupling of aryl bromides and aniline derivatives (Method A).}

$\mathrm{Pd}_{2}(\mathrm{dba})_{3}(2-4 \mu \mathrm{mol})$, DTBNpP $(10 \%$ solution in toluene) or DTBNpP·HBF 4 - $(4-8 \mu \mathrm{mol}, 1$ eq/Pd), sodium $t$-butoxide $(0.85 \mathrm{mmol})$, and toluene $(2 \mathrm{~mL})$ were added to a round bottom flask in a glove box. Upon removing the septum sealed flask from the drybox, the aryl bromide (0.80 mmol) and aniline derivative ( $\mathbf{8}$ or $\mathbf{1 0}, 0.82-1.0 \mathrm{mmol}$ ) were added by syringe and the reaction was allowed to stir for 1-6 hours at room temperature until judged complete by GC. The reaction mixture was then adsorbed on silica gel and purified by flash chromatography.

\section{Typical procedure for the coupling of aryl chlorides and aniline derivatives (Method B).} $\mathrm{Pd}_{2}(\mathrm{dba})_{3}(2-20 \mu \mathrm{mol})$, DTBNpP $(10 \%$ solution in toluene) or DTBNpP·HBF $4 .(4-40 \mu \mathrm{mol}, 1$ eq/Pd), sodium $t$-butoxide $(0.85 \mathrm{mmol})$, and solvent (toluene or xylenes) $(2 \mathrm{~mL})$ were added to a round bottom flask in a glove box. Upon removing the septum sealed flask from the drybox, the aryl chloride $(0.80 \mathrm{mmol})$ and aniline derivative (8 or $\mathbf{1 0}, 0.82-1.0 \mathrm{mmol})$ were added by syringe. The flask was placed in a preheated oil bath at the indicated temperature and stirred for 2-24 hours until judged complete by GC. The reaction mixture was then adsorbed on silica gel and purified by flash chromatography. 
$N$-(4-Fluorophenyl)aniline (Table 3, entry 1). 1-Bromo-4-fluorobenzene (140 mg, 0.80 mmol) and aniline (93 mg, $1.0 \mathrm{mmol})$ were coupled using $\mathrm{Pd}_{2}\left(\mathrm{dba}_{3}(3.7 \mathrm{mg}, 4.0 \mu \mathrm{mol})\right.$ and DTBNpP·HBF 4 (2.3 mg. $8.0 \mu \mathrm{mol})$ according to Method A. The crude product was purified by column chromatography (5\% ethyl acetate/95\% hexanes) to afford $N$-(4-fluorophenyl)aniline (84\%, $126 \mathrm{mg}$ ) as a low-melting solid. ${ }^{1} \mathrm{H}$ NMR (500 MHz, $\mathrm{CDCl}_{3}$ ): $\delta 7.26$ (dd, 7.27, 8.48 Hz, 2H), 7.04-7.07 (m, 2H), 6.97-7.01 (m, 4H), 6.91 (tt, 1.11, $7.44 \mathrm{~Hz}, 1 \mathrm{H}), 5.58$ (brs, $1 \mathrm{H}) .{ }^{13} \mathrm{C}$ NMR (90.6 MHz, DMSO): $\delta 155.9(\mathrm{~d}, J=235.5 \mathrm{~Hz}), 143.5,139.3\left(\mathrm{~d}, J_{\mathrm{C}-\mathrm{F}}=2.67\right), 128.7,118.9$, $118.4\left(\mathrm{~d}, J_{\mathrm{C}-\mathrm{F}}=7.34\right), 115.6,115.1\left(\mathrm{~d}, J_{\mathrm{C}-\mathrm{F}}=22.0\right)$. The ${ }^{1} \mathrm{H}$ NMR spectrum was consistent with previously reported data. ${ }^{1}$

$N$-(4-Trifluoromethylphenyl)aniline (Table 3, entry 2). 4-Bromobenzotrifluoride (179 mg, $0.80 \mathrm{mmol})$ and aniline (93 mg, $1.0 \mathrm{mmol})$ were coupled using $\mathrm{Pd}_{2}(\mathrm{dba})_{3}(3.7 \mathrm{mg}, 4.0 \mu \mathrm{mol})$ and DTBNpP·HBF 4 (2.3 mg. $8.0 \mu \mathrm{mol})$ according to Method A. The crude product was purified by column chromatography (5\% ethyl acetate/95\% hexanes) to afford $N$-(4-(trifluoromethyl)phenyl)aniline (96\%, $182 \mathrm{mg}) .{ }^{1} \mathrm{H}$ NMR (500 MHz, DMSO- $\left.d_{6}\right): \delta 8.68(\mathrm{~s}, 1 \mathrm{H}), 7.50$ (d, $J=$ $8.83 \mathrm{~Hz}, 2 \mathrm{H}), 7.31$ (t, $J=7.97 \mathrm{~Hz}, 2 \mathrm{H}), 7.16$ (d, $J=7.50 \mathrm{~Hz}, 2 \mathrm{H}), 7.13$ (d, $J=8.45 \mathrm{~Hz}, 2 \mathrm{H}), 6.97$ (t, $J=7.25 \mathrm{~Hz}, 1 \mathrm{H}) .{ }^{13} \mathrm{C}$ NMR $\left(90.6 \mathrm{MHz}, \mathrm{DMSO}-d_{6}\right): \delta 148.0,142.0,129.8,126.9\left(\mathrm{q}, J_{\mathrm{C}-\mathrm{F}}=\right.$ 3.71 Hz), $125.4\left(\mathrm{q}, J_{\mathrm{C}-\mathrm{F}}=270.4 \mathrm{~Hz}\right), 122.1,119.5,119\left(\mathrm{q}, J_{\mathrm{C}-\mathrm{F}}=32.1 \mathrm{~Hz}\right), 115.1$. The NMR spectral data was consistent with the previously reported data. ${ }^{2}$ mp: $63-65^{\circ} \mathrm{C}$ (lit. mp: $63-64$ $\left.{ }^{\circ} \mathrm{C}\right){ }^{3}$

$N$-Phenyl-p-toluidine (Table 3, entry 3; Table 6, entry 1). 4-Bromotoluene (138 mg, 0.80 mmol) and aniline (93 mg, $1.0 \mathrm{mmol})$ were coupled using $\mathrm{Pd}_{2}(\mathrm{dba})_{3}(3.7 \mathrm{mg}, 4.0 \mu \mathrm{mol})$ and DTBNpP·HBF 4 (2.3 mg. $8.0 \mu \mathrm{mol})$ according to Method A. The crude product was purified by column chromatography (5\% ethyl acetate/95\% hexanes) to afford $N$-phenyl-p-toluidine (125 
mg, 86\%). ${ }^{1} \mathrm{H}$ NMR (500 MHz, DMSO- $\left.d_{6}\right): \delta 7.21$ (m, 5H), 6.82 (t, $\left.J=7.60 \mathrm{~Hz}, 1 \mathrm{H}\right), 6.59(\mathrm{~d}, J$ $=7.88 \mathrm{~Hz}, 2 \mathrm{H}), 5.24(\mathrm{~s}, 1 \mathrm{H}), 2.31(\mathrm{~s}, 6 \mathrm{H}) .{ }^{13} \mathrm{C}$ NMR (90.6 MHz, DMSO-d 6 ): $\delta$ 146.5, 138.4, 136.1, 129.4, 128.7, 125.9, 118.4, 113.7, 18.5. The NMR spectral data was consistent with previously reported data. ${ }^{4} \mathrm{mp}: 87^{\circ} \mathrm{C}$ (lit. mp: $\left.87-88{ }^{\circ} \mathrm{C}\right) .^{5}$

Alternatively, 4-Chlorotoluene (101 mg, $0.80 \mathrm{mmol}$ ) and aniline (93 mg, $1.0 \mathrm{mmol}$ ) were coupled using $\mathrm{Pd}_{2}(\mathrm{dba})_{3}(3.7 \mathrm{mg}, 4.0 \mu \mathrm{mol})$ and $\mathrm{DTBNpP} \mathrm{HBF}_{4}(2.3 \mathrm{mg} .8 .0 \mu \mathrm{mol})$ according to Method B at $120^{\circ} \mathrm{C}$ in xylenes to afford $120 \mathrm{mg}$ of $N$-phenyl-p-toluidine (86\%).

$N$-Phenyl-p-anisidine (Table 3, entry 4; Table 6, entry 2). 4-Bromoanisole (150 mg, 0.80 mmol) and aniline (93 mg, $1.0 \mathrm{mmol})$ were coupled using $\mathrm{Pd}_{2}(\mathrm{dba})_{3}(3.7 \mathrm{mg}, 4.0 \mu \mathrm{mol})$ and DTBNpP·HBF 4 (2.3 mg. $8.0 \mu \mathrm{mol})$ according to Method A. The crude product was purified by column chromatography (5\% ethyl acetate/95\% hexanes) to afford $N$-phenyl-p-anisidine (92\%, $138 \mathrm{mg}) .{ }^{1} \mathrm{H}$ NMR (500 MHz, $\left.\mathrm{CDCl}_{3}\right): \delta 7.26(\mathrm{~d}, J=7.89 \mathrm{~Hz}, 2 \mathrm{H}), 7.12(\mathrm{~d}, J=8.82 \mathrm{~Hz}, 2 \mathrm{H})$, $6.96(\mathrm{~d}, J=7.64 \mathrm{~Hz}, 2 \mathrm{H}), 6.90$ (m, 3H), $5.53(\mathrm{~s}, 1 \mathrm{H}), 3.85$ (s, 3H). ${ }^{13} \mathrm{C} \mathrm{NMR}(90.6 \mathrm{MHz}$, $\left.\mathrm{CDCl}_{3}\right): \delta 155.2,145.1,135.7,129.3,122.2,119.5,115.6,114.6,55.5$. The NMR spectral data was consistent with previously reported data. ${ }^{6} \mathrm{mp}: 98-100{ }^{\circ} \mathrm{C}$ (lit. mp: $\left.102-103^{\circ} \mathrm{C}\right) .^{7}$

Alternatively, 4-Chloroanisole (114 mg, $0.80 \mathrm{mmol}$ ) and aniline (93 mg, $1.0 \mathrm{mmol}$ ) were coupled using $\mathrm{Pd}_{2}(\mathrm{dba})_{3}(3.7 \mathrm{mg}, 4.0 \mu \mathrm{mol})$ and DTBNpP·HBF $4(2.3 \mathrm{mg} .8 .0 \mu \mathrm{mol})$ according to Method B at $140{ }^{\circ} \mathrm{C}$ in xylenes to afford $141 \mathrm{mg}$ of $N$-phenyl-p-anisidine (89\%).

Bis(4-methoxyphenyl)amine (Table 3, entry 5). 4-Bromoanisole (150 mg, $0.80 \mathrm{mmol}$ ) and anisidine (126 mg, $1.04 \mathrm{mmol})$ were coupled using $\mathrm{Pd}_{2}(\mathrm{dba})_{3}(3.7 \mathrm{mg}, 4.0 \mu \mathrm{mol})$ and DTBNpP·HBF 4 (2.3 mg. $8.0 \mu \mathrm{mol}$ ) according to Method A. The crude product was purified by column chromatography (methylene chloride) to afford bis(4-methoxyphenyl)amine (92\%, 138 mg). ${ }^{1} \mathrm{H}$ NMR (500 MHz, DMSO-d $): \delta 7.50$ (s,1H), $6.91(\mathrm{~d}, J=8.94 \mathrm{~Hz}, 4 \mathrm{H}), 6.80(\mathrm{~d}, J=8.93$ 
$\mathrm{Hz}, 4 \mathrm{H}), 3.68$ (s, 6H). ${ }^{13} \mathrm{C}$ NMR (90.6 MHz, DMSO-d $\left.d_{6}\right): \delta 152.7,138.9,117.9,114.4,55.1$. mp: $98^{\circ} \mathrm{C}$ (lit. mp: $\left.96.8^{\circ} \mathrm{C}\right) .{ }^{8}$

$N, N$-Dimethyl- $N$ '-phenyl-1,4-benzenediamine (Table 3, entry 6). 4-Bromo- $N, N$ dimethylaniline (159 mg, $0.78 \mathrm{mmol}$ ) and aniline (93 mg, $1.0 \mathrm{mmol}$ ) were coupled using $\mathrm{Pd}_{2}(\mathrm{dba})_{3}(3.7 \mathrm{mg}, 4.0 \mu \mathrm{mol})$ and DTBNpP·HBF $4(2.3 \mathrm{mg} .8 .0 \mu \mathrm{mol})$ according to Method A. The crude product was purified by column chromatography (5\% ethyl acetate/95\% hexanes) to afford $N, N$-dimethyl- $N$--phenylbenzene-1,4-diamine (78\%, $129 \mathrm{mg}) .{ }^{1} \mathrm{H}$ NMR (500 MHz, $\left.\mathrm{C}_{6} \mathrm{D}_{6}\right): \delta$ 7.11-7.15 (m, 2H), 6.98 (d, $\left.8.93 \mathrm{~Hz}, 2 \mathrm{H}\right), 6.77-6.81$ (m, 3H), 6.57 (d, $\left.8.93 \mathrm{~Hz}, 2 \mathrm{H}\right)$, 5.21 (brs, $1 \mathrm{H}), 2.53$ (s, 6H). ${ }^{13} \mathrm{C}$ NMR (90.6 MHz, $\left.\mathrm{CDCl}_{3}\right): \delta$ 147.0, 146.0, 132.1, 128.9, 123.0, 118.5, 114.7, 113.7, 40.9, 29.4. The NMR spectral data was consistent with previously reported data. ${ }^{9} \mathrm{mp}: 115^{\circ} \mathrm{C}$.

$N$-Phenyl-o-toluidine (Table 3, entry 7; Table 6, entry 3). 2-Bromotoluene (137 mg, 0.80 mmol) and aniline (93 mg, $1.0 \mathrm{mmol})$ were coupled using $\mathrm{Pd}_{2}(\mathrm{dba})_{3}(3.7 \mathrm{mg}, 4.0 \mu \mathrm{mol})$ and DTBNpP·HBF 4 (2.3 mg. $8.0 \mu \mathrm{mol})$ according to Method A. The crude product was purified by column chromatography (5\% ethyl acetate/95\% hexanes) to afford $N$-phenyl-o-toluidine (117.1 mg, 80\%) as an oil. ${ }^{1} \mathrm{H}$ NMR (500 MHz, $\left.\mathrm{CDCl}_{3}\right): \delta 7.32(\mathrm{t}, J=8.00 \mathrm{~Hz}, 3 \mathrm{H}), 7.28(\mathrm{~d}, J=7.46$ Hz, 1H), 7.21 (t, $J=7.54 \mathrm{~Hz}, 1 \mathrm{H}), 7.03$ (m, 3H), 6.98 (t, $J=7.94 \mathrm{~Hz}, 1 \mathrm{H}), 5.44$ (s, 1H), 2.33 (s, 3H). ${ }^{13} \mathrm{C}$ NMR (90.6 MHz, $\left.\mathrm{CDCl}_{3}\right): \delta 144.0,141.2,130.9,129.3,128.3,126.7,122.0,120.4$, 118.9, 117.4, 17.8. The NMR spectral data was consistent with previously reported data. ${ }^{10}$ Alternatively, 2-Chlorotoluene (102 mg, $0.80 \mathrm{mmol}$ ) and aniline (93 mg, $1.0 \mathrm{mmol}$ ) were coupled using $\mathrm{Pd}_{2}(\mathrm{dba})_{3}(3.7 \mathrm{mg}, 4.0 \mu \mathrm{mol})$ and $\mathrm{DTBNpP} \cdot \mathrm{HBF}_{4}(2.3 \mathrm{mg} .8 .0 \mu \mathrm{mol})$ according to Method B at $120^{\circ} \mathrm{C}$ in xylenes to afford $139 \mathrm{mg}$ of $N$-phenyl-o-toluidine (95\%). 
2,6-Dimethyl- $N$-phenylaniline (Table 3, entry 8). 2-Bromo- $m$-xylene (148 mg, $0.80 \mathrm{mmol}$ ) and aniline (93 mg, $1.0 \mathrm{mmol})$ were coupled using $\mathrm{Pd}_{2}(\mathrm{dba})_{3}(3.7 \mathrm{mg}, 4.0 \mu \mathrm{mol})$ and DTBNpP·HBF 4 (2.3 mg. $8.0 \mu \mathrm{mol})$ according to Method A. The crude product was purified by column chromatography (5\% ethyl acetate/95\% hexanes) to afford 2,6-dimethyl- $N$-phenylaniline (122.1 mg, 77\%) as an oil. ${ }^{1} \mathrm{H}$ NMR (500 MHz, $\left.\mathrm{CDCl}_{3}\right): \delta 7.21(\mathrm{~m}, 5 \mathrm{H}), 6.82(\mathrm{t}, J=7.60,1 \mathrm{H})$, 6.59 (d, $J=7.88,2 \mathrm{H}), 5.24(\mathrm{~s}, 1 \mathrm{H}), 2.31(\mathrm{~s}, 6 \mathrm{H}) .{ }^{13} \mathrm{C} \mathrm{NMR}\left(90.6 \mathrm{MHz}, \mathrm{CDCl}_{3}\right): \delta 146.5,138.4$, 136.1, 129.4, 128.7, 125.9, 118.4, 113.7, 18.5. The NMR spectral data was consistent with previously reported data. ${ }^{10}$

2,4,6-Trimethyl- $N$-(4-methylphenyl)aniline (Table 3, entry 9). 4-Bromotoluene (137 mg, $0.80 \mathrm{mmol}$ ) and 2,4,6-trimethylaniline (135 mg, $1.0 \mathrm{mmol})$ were coupled using $\operatorname{Pd}_{2}(\mathrm{dba})_{3}(3.7$ mg, $4.0 \mu \mathrm{mol})$ and DTBNpP·HBF $4(2.3 \mathrm{mg}$. $8.0 \mu \mathrm{mol})$ according to Method A. The crude product was purified by column chromatography (5\% ethyl acetate/95\% hexanes) to afford 2,4,6trimethyl- $N$-(4-methylphenyl)aniline (60\%, $106 \mathrm{mg}) .{ }^{1} \mathrm{H}$ NMR (500 MHz, DMSO- $\left.d_{6}\right): \delta 6.96$ (s, 1H), 6.90 (s, 2H), 6.85 (d, $J=8.13 \mathrm{~Hz}, 2 \mathrm{H}), 6.28$ (d, $J=8.12 \mathrm{~Hz}, 2 \mathrm{H}), 2.23$ (s, 3H), 2.13 (s, 3H), 2.07 (s, 6H). ${ }^{13} \mathrm{C}$ NMR (90.6 MHz, DMSO- $\left.d_{6}\right): \delta 144.9,136.2,135.3,133.9,129.3,128.8$, 124.6, 112.3, 20.4, 20.0, 17.9. The ${ }^{1} \mathrm{H}$ NMR spectrum was consistent with previously reported data. ${ }^{11} \mathrm{mp}: 61-63^{\circ} \mathrm{C}$ (lit. mp: $\left.66-67^{\circ} \mathrm{C}\right) .{ }^{11}$

2,6-Diisopropyl- $N$-(4-methylphenyl)aniline (Table 3, entry 10). ${ }^{12}$ 4-Bromotoluene (137 mg, $0.80 \mathrm{mmol}$ ) and 2,6-diisopropylaniline (177 mg, $1.0 \mathrm{mmol})$ were coupled using $\mathrm{Pd}_{2}(\mathrm{dba})_{3}$ (3.7 mg, $4.0 \mu \mathrm{mol}$ ) and DTBNpP·HBF 4 (2.3 mg. $8.0 \mu \mathrm{mol})$ according to Method A. The crude product was purified by column chromatography (5\% ethyl acetate/95\% hexanes) to afford 109 mg of crude 2,6-diisopropyl- $N$-(4-methylphenyl)aniline contaminated with $8 \%$ (w/w) of 2,6diisopropylamine that could not be removed. The yield of 2,6-diisopropyl- $N$-(4- 
methylphenyl)aniline was estimated to be $100 \mathrm{mg}(47 \%) .{ }^{1} \mathrm{H}$ NMR (500 MHz, DMSO- $\left.d_{6}\right): \delta$ 7.21 (m, 3H), 7.05 (s, 1H), 6.83 (d, $J=8.09 \mathrm{~Hz}, 2 \mathrm{H}), 6.30$ (d, $J=7.56 \mathrm{~Hz}, 2 \mathrm{H}), 3.17$ (sept, $J=$ $6.94 \mathrm{~Hz}, 2 \mathrm{H}), 2.13$ (s, 3H), 1.06 (d, $J=6.96 \mathrm{~Hz}, 12 \mathrm{H}) .{ }^{13} \mathrm{C}$ NMR $\left(90.6 \mathrm{MHz}, \mathrm{DMSO}-d_{6}\right): \delta$ 147.7, 147.1, 136.3, 129.8, 127.1, 124.2, 123.9, 112.4, 28.0, 24.2, 20.5.

2,4,6-Trimethyl- $N$-(2-methylphenyl)aniline (Table 3, entry 11). 2-Bromotoluene (120 mg, $0.80 \mathrm{mmol}$ ) and 2,4,6-trimethylaniline (135 mg, $1.0 \mathrm{mmol})$ were coupled using $\operatorname{Pd}_{2}(\mathrm{dba})_{3}(3.7$ mg, $4.0 \mu \mathrm{mol})$ and DTBNpP·HBF $4(2.3 \mathrm{mg} .8 .0 \mu \mathrm{mol})$ according to Method A. The crude product was purified by column chromatography (5\% ethyl acetate/95\% hexanes) to afford 2,4,6trimethyl- $N$-(2-methylphenyl)aniline (78\%, $141 \mathrm{mg}) .{ }^{1} \mathrm{H}$ NMR (500 MHz, DMSO- $\left.d_{6}\right): \delta 7.01$ (d, $J=6.97 \mathrm{~Hz}, 1 \mathrm{H}), 6.94(\mathrm{~s}, 2 \mathrm{H}), 6.82(\mathrm{t}, J=7.71 \mathrm{~Hz}, 1 \mathrm{H}), 6.51$ (t, $J=7.75 \mathrm{~Hz}, 1 \mathrm{H}), 6.34$ (s, 1H), $5.84(\mathrm{~d}, J=7.78 \mathrm{~Hz}, 1 \mathrm{H}), 2.26$ (s, 6H), $2.06(\mathrm{~s}, 6 \mathrm{H}) .{ }^{13} \mathrm{C}$ NMR $\left(90.6 \mathrm{MHz}\right.$, DMSO- $\left.d_{6}\right): \delta$ 144.8, 136.1, 135.6, 134.0, 129.8, 128.6, 126.2, 121.6, 116.1, 109.9, 20.3, 17.6, 17.5. The ${ }^{1} \mathrm{H}$ NMR spectrum was consistent with previously reported data. ${ }^{11} \mathrm{mp}$ 76-78 ${ }^{\circ} \mathrm{C}$ (lit. mp: 78.5-79.5 $\left.{ }^{\circ} \mathrm{C}\right) .{ }^{11}$

2,4,6-Trimethyl- $N$-(2,6-dimethylphenyl)aniline (Table 3, entry 12). 2-Bromo- $m$-xylene (135 mg, $0.80 \mathrm{mmol}$ ) and 2,4,6-trimethylaniline (135 mg, $1.0 \mathrm{mmol}$ ) were coupled using $\mathrm{Pd}_{2}(\mathrm{dba})_{3}(7.4 \mathrm{mg}, 8.0 \mu \mathrm{mol})$ and DTBNpP$\cdot \mathrm{HBF}_{4}(4.6 \mathrm{mg} .16 \mu \mathrm{mol})$ according to Method A. The reaction was heated at $50^{\circ} \mathrm{C}$. The crude product was purified by column chromatography (5\% ethyl acetate/95\% hexanes) to afford 2,4,6-trimethyl- $N$-(2,6-dimethylphenyl)aniline (75\%, $143 \mathrm{mg}) .{ }^{1} \mathrm{H}$ NMR (500 MHz, DMSO- $\left.d_{6}\right): \delta 6.88(\mathrm{~d}, J=7.21 \mathrm{~Hz}, 2 \mathrm{H}), 6.74(\mathrm{~s}, 2 \mathrm{H}), 6.69$ (t, $J=$ $7.41 \mathrm{~Hz}, 1 \mathrm{H}), 5.99$ (s, $1 \mathrm{H}), 2.17$ (s, 3H), 1.91 (s, 12H). ${ }^{13} \mathrm{C}$ NMR (90.6 MHz, DMSO-d $\left.d_{6}\right): \delta$ 143.3, 140.2, 130.9, 130.6, 128.3, 128.9, 128.8, 120.8, 20.7, 19.5, 19.4. The ${ }^{1} \mathrm{H}$ NMR spectrum was consistent with previously reported data. ${ }^{13} \mathrm{mp} 97^{\circ} \mathrm{C}$ (lit. mp: $97-99{ }^{\circ} \mathrm{C}$ ). ${ }^{13}$ 


\section{4-( $N$-Methyl- $N$-phenylamino)benzonitrile (Table 4, entry 1; Table 6, entry 4). 4-}

Bromobenzonitrile (146 mg, $0.80 \mathrm{mmol}$ ) and $N$-methylaniline $(88.0 \mathrm{mg}, 0.82 \mathrm{mmol}$ ) were coupled using $\mathrm{Pd}_{2}(\mathrm{dba})_{3}(1.9 \mathrm{mg}, 2.0 \mu \mathrm{mol})$ and DTBNpP·HBF $4(1.2 \mathrm{mg} .4 \mu \mathrm{mol})$ according to Method A. The crude product was purified by column chromatography (10\% ethyl acetate/90\% hexanes) to afford 4-( $N$-methyl- $N$-phenylamino)benzonitrile (165 mg, 99\%) as a pale yellow oil. ${ }^{1} \mathrm{H}$ NMR (500 MHz, DMSO-d $\left._{6}\right): \delta 7.46$ (m, 4H), 7.25 (m, 3H), 6.76 (d, $\left.J=9.31 \mathrm{~Hz}, 2 \mathrm{H}\right), 3.38$ (s, 3H). ${ }^{13} \mathrm{C}$ NMR (90.6 MHz, DMSO- $\left.d_{6}\right): \delta 152.0,146.8,133.2,130.1,126.4,126.2,120.3$, 113.9, 99.4, 40.2. The NMR spectral data was identical to previously reported data. ${ }^{14}$

Alternatively, 4-Chlorobenzonitrile (110 mg, $0.80 \mathrm{mmol}$ ) and $N$-methylaniline (88.0 mg, 0.82 mmol) were coupled using $\mathrm{Pd}_{2}(\mathrm{dba})_{3}(1.9 \mathrm{mg}, 2.0 \mu \mathrm{mol})$ and DTBNpP·HBF $4(1.2 \mathrm{mg} .4 .0 \mu \mathrm{mol})$ according to Method B at $50{ }^{\circ} \mathrm{C}$ in toluene to afford $160 \mathrm{mg}$ of 4-( $N$-methyl- $N$ phenylamino)benzonitrile (97\%).

$N$-(4-Fluorophenyl)- $N$-methylaniline (Table 4, entry 2). 1-Bromo-4-fluorobenzene (140 mg, $0.80 \mathrm{mmol})$ and $N$-methylaniline $(88.0 \mathrm{mg}, 0.82 \mathrm{mmol})$ were coupled using $\mathrm{Pd}_{2}(\mathrm{dba})_{3}(1.9$ mg, $2.0 \mu \mathrm{mol})$ and DTBNpP·HBF $4(1.2 \mathrm{mg} .4 \mu \mathrm{mol})$ according to Method A. The crude product was purified by column chromatography (5\% ethyl acetate/95\% hexanes) to afford $N$-(4fluorophenyl)- $N$-methylaniline (149 mg, 93\%) as a light yellow oil. ${ }^{1} \mathrm{H}$ NMR (500 MHz, $\left.\mathrm{CDCl}_{3}\right): \delta 7.22$ (dd, $\left.J=8.83,7.27 \mathrm{~Hz}, 2 \mathrm{H}\right), 7.00(\mathrm{~m}, 4 \mathrm{H}), 6.87$ (m, 3H), 3.25 (s, 3H). ${ }^{13} \mathrm{C}$ NMR (90.6 MHz, $\left.\mathrm{CDCl}_{3}\right): \delta 158.7\left(\mathrm{~d}, J_{\mathrm{C}-\mathrm{F}}=242.0 \mathrm{~Hz}\right), 149.4,145.3\left(\mathrm{~d}, J_{\mathrm{C}-\mathrm{F}}=2.79 \mathrm{~Hz}\right), 129.2,124.2$, 124.4, 120.1, 118.1, $116.0\left(\mathrm{~d} J_{\mathrm{C}-\mathrm{F}}=20.2 \mathrm{~Hz}\right), 40.6$.

$N$-(4-Trifluoromethylphenyl)- $N$-methylaniline (Table 4, entry 3). 4-Bromobenzotrifluoride (180 mg, $0.80 \mathrm{mmol}$ ) and $N$-methylaniline $\left(88.0 \mathrm{mg}, 0.82 \mathrm{mmol}\right.$ ) were coupled using $\operatorname{Pd}_{2}(\mathrm{dba})_{3}$ (1.9 mg, $2.0 \mu \mathrm{mol})$ and DTBNpP·HBF $4(1.2 \mathrm{mg} .4 \mu \mathrm{mol})$ according to Method A. The crude 
product was purified by column chromatography (5\% ethyl acetate/95\% hexanes) to afford $\mathrm{N}$-(4trifluoromethylphenyl)- $N$-methylaniline (189 $\mathrm{mg}, 93 \%$ ) as a light yellow oil. ${ }^{1} \mathrm{H}$ NMR (500 MHz, $\left.\mathrm{CDCl}_{3}\right): \delta 7.45(\mathrm{~m}, 4 \mathrm{H}), 7.23(\mathrm{~m}, 3 \mathrm{H}), 6.88(\mathrm{~d}, J=8.49 \mathrm{~Hz}, 2 \mathrm{H}), 6.82(\mathrm{~m}, 3 \mathrm{H}), 3.39(\mathrm{~s}$, 3H). ${ }^{13} \mathrm{C}$ NMR $\left(90.6 \mathrm{MHz}, \mathrm{CDCl}_{3}\right): \delta 151.5,147.8,129.8,126.2,125.3,125.0,122.5\left(\mathrm{q}, J_{\mathrm{C}-\mathrm{F}}=\right.$ $269 \mathrm{~Hz}), 119.8,114.8,40.2$. The NMR spectral data was identical to previously reported data. ${ }^{15}$

$N$-Methyl- $N$-phenyl-p-toluidine (Table 4, entry 4; Table 6, entry 5). 4-Bromotoluene (138 mg, $0.80 \mathrm{mmol})$ and $N$-methylaniline $(88.0 \mathrm{mg}, 0.82 \mathrm{mmol})$ were coupled using $\mathrm{Pd}_{2}\left(\mathrm{dba}_{3}\right)_{3}(1.9$ mg, $2.0 \mu \mathrm{mol})$ and DTBNpP·HBF $4(1.2 \mathrm{mg} .4 \mu \mathrm{mol})$ according to Method A. The crude product was purified by column chromatography (2.5\% ethyl acetate/97.5\% hexanes) to afford $N$-methyl$N$-phenyl-p-toluidine (148 mg, 93\%) as a light yellow oil. ${ }^{1} \mathrm{H}$ NMR (500 MHz, $\mathrm{CDCl}_{3}$ ): $\delta 7.31$ (t, $J=8.51 \mathrm{~Hz}, 2 \mathrm{H}), 7.19$ (d, $J=8.57 \mathrm{~Hz}, 2 \mathrm{H}), 7.07$ (d, $J=8.08 \mathrm{~Hz}, 2 \mathrm{H}), 7.00$ (d, $J=8.59 \mathrm{~Hz}$, 2H), 6.94 (t, $J=7.38 \mathrm{~Hz}, 1 \mathrm{H}), 3.36$ (s, 3H), 2.40 (s, 3H). ${ }^{13} \mathrm{C} \mathrm{NMR}\left(90.6 \mathrm{MHz}, \mathrm{CDCl}_{3}\right): \delta$ 149.4, 146.7, 132.0, 129.9, 129.1, 122.6, 119.8, 118.2, 40.4, 20.8. The NMR spectral data was identical to literature values. ${ }^{10}$

Alternatively, 4-Chlorotoluene (101 mg, $0.80 \mathrm{mmol}$ ) and $N$-methylaniline (88.0 mg, 0.82 mmol) were coupled using $\mathrm{Pd}_{2}(\mathrm{dba})_{3}(1.9 \mathrm{mg}, 2.0 \mu \mathrm{mol})$ and DTBNpP·HBF $4(1.2 \mathrm{mg} .4 .0 \mu \mathrm{mol})$ according to Method B at $100{ }^{\circ} \mathrm{C}$ in toluene to afford $109 \mathrm{mg}$ of $N$-methyl- $N$-phenyl-p-toluidine (70\%).

$N$-Methyl- $N$-phenyl-p-anisidine (Table 4, entry 5; Table 6, entry 6). 4-Bromoanisole (138 mg, $0.80 \mathrm{mmol})$ and $N$-methylaniline $(88.0 \mathrm{mg}, 0.82 \mathrm{mmol})$ were coupled using $\operatorname{Pd}_{2}\left(\mathrm{dba}_{3}(1.9\right.$ mg, $2.0 \mu \mathrm{mol})$ and DTBNpP·HBF 4 (1.2 mg. $4 \mu \mathrm{mol})$ according to Method A. The crude product was purified by column chromatography (5\% ethyl acetate/95\% hexanes) to afford $N$-methyl- $N$ phenyl-p-anisidine (148 mg, 93\%) as a light yellow oil. ${ }^{1} \mathrm{H}$ NMR (500 MHz, $\mathrm{CDCl}_{3}$ ): $\delta 7.25$ 
(m, 2H), 7.13 (d, $J=9.01 \mathrm{~Hz}, 2 \mathrm{H}), 6.93$ (d, $J=9.17 \mathrm{~Hz}, 2 \mathrm{H}), 6.82$ (m, 3H), 3.85 (s, 3H), 3.30 (s,

3H). ${ }^{13} \mathrm{C}$ NMR (90.6 MHz, $\left.\mathrm{CDCl}_{3}\right): \delta 156.3,149.8,142.3,129.0,126.2,118.4,115.8,114.8$,

55.5, 40.5. The NMR spectral data was identical to literature values. ${ }^{10}$

Alternatively, 4-Chloroanisole (114 mg, $0.80 \mathrm{mmol}$ ) and $N$-methylaniline ( $88.0 \mathrm{mg}, 0.82$

mmol) were coupled using $\mathrm{Pd}_{2}(\mathrm{dba})_{3}(19 \mathrm{mg}, 20 \mu \mathrm{mol})$ and DTBNpP·HBF $4(12 \mathrm{mg} .40 \mu \mathrm{mol})$

according to Method B at $100{ }^{\circ} \mathrm{C}$ in toluene for 18 hours to afford $168 \mathrm{mg}$ of $\mathrm{N}$-methyl- $\mathrm{N}$ -

phenyl-p-anisidine (99\%).

$N, N, N^{\prime}$-Trimethyl- $N$ '-phenyl-1,4-benzenediamine (Table 4, entry 6). 4-Bromo- $N, N$ dimethylaniline (160 mg, $0.80 \mathrm{mmol}$ ) and $N$-methylaniline (88 mg, $1.0 \mathrm{mmol}$ ) were coupled using $\mathrm{Pd}_{2}(\mathrm{dba})_{3}(1.9 \mathrm{mg}, 2.0 \mu \mathrm{mol})$ and DTBNpP$\cdot \mathrm{HBF}_{4}(1.2 \mathrm{mg} .4 \mu \mathrm{mol})$ according to Method A. The crude product was purified by column chromatography (25\% methylene chloride/75\% hexanes) to afford $N, N, N^{\prime}$-trimethyl- $N^{\prime}$-phenyl-1,4-benzenediamine (163 mg, 89\%) as a pale yellow oil. ${ }^{1} \mathrm{H}$ NMR (500 MHz, $\left.\mathrm{CDCl}_{3}\right): \delta 7.25(\mathrm{t}, J=7.25 \mathrm{~Hz}, 2 \mathrm{H}), 7.15$ (d, $\left.J=8.80 \mathrm{~Hz}, 2 \mathrm{H}\right)$, 6.82 (m, 5H), 3.32 (s, 3H), 3.02 (s, 6H). ${ }^{13} \mathrm{C}$ NMR (90.6 MHz, $\left.\mathrm{CDCl}_{3}\right): \delta$ 150.1, 148.1, 138.8, 128.9, 126.9, 117.5, 114.7, 113.8, 41.0, 40.5. The NMR spectral data was identical to literature values. ${ }^{16}$

$N$-Methyl- $N$-phenyl-o-toluidine (Table 4, entry 7). 2-Bromotoluene (138 mg, $0.80 \mathrm{mmol}$ ) and $N$-methylaniline (88 mg, $1.0 \mathrm{mmol})$ were coupled using $\mathrm{Pd}_{2}(\mathrm{dba})_{3}(1.9 \mathrm{mg}, 2.0 \mu \mathrm{mol})$ and DTBNpP·HBF 4 (1.2 mg. $4 \mu \mathrm{mol})$ according to Method A. The crude product was purified by column chromatography (2.5\% ethyl acetate/97.5\% hexanes) to afford $N$-methyl- $N$-phenyl-otoluidine (141 mg, 90\%) as a pale yellow oil. ${ }^{1} \mathrm{H}$ NMR (500 MHz, $\left.\mathrm{CDCl}_{3}\right): \delta 7.17(\mathrm{~m}, 6 \mathrm{H}), 6.69$ (t, J = 7.28 Hz, 1H), 6.52 (d, J = $7.83 \mathrm{~Hz}, 2 \mathrm{H}), 3.21$ (s, 3H), 2.13 (s, 3H). ${ }^{13} \mathrm{C}$ NMR (90.6 MHz, 
$\left.\mathrm{CDCl}_{3}\right): \delta 149.2,146.9,136.9,131.4,129.0,128.4,127.6,126.5,116.9,112.9,39.1,17.9$. The NMR spectral data was identical to literature values. ${ }^{15}$

$N, N$-Diphenyl-p-toluidine (Table 4, entry 9). 4-Bromotoluene (138 mg, $0.80 \mathrm{mmol}$ ) and diphenylamine (132 mg, $0.78 \mathrm{mmol})$ were coupled using $\mathrm{Pd}_{2}(\mathrm{dba})_{3}(7.3 \mathrm{mg}, 8.0 \mu \mathrm{mol})$ and DTBNpP·HBF 4 (4.9 mg. $16 \mu \mathrm{mol})$ according to Method A. The crude product was purified by column chromatography (5\% ethyl acetate/95\% hexanes) to afford $N, N$-diphenyl-p-toluidine (200 mg, 97\%) as a white solid. ${ }^{1} \mathrm{H}$ NMR (500 MHz, $\left.\mathrm{CDCl}_{3}\right)$ : $\delta 7.25$ (m, 4H), 7.09 (m, 6H), 7.02 (m, 4H), 2.34 (s, 3H). ${ }^{13} \mathrm{C}$ NMR (90.6 MHz, $\left.\mathrm{CDCl}_{3}\right): \delta 148.1,145.3,132.7,129.9,129.1$, 124.9, 123.6, 122.2, 20.8. The NMR spectral data was identical to literature values. ${ }^{10} \mathrm{mp}: 67-68$ ${ }^{\circ} \mathrm{C}$ (lit. mp: $\left.69-70{ }^{\circ} \mathrm{C}\right) .{ }^{17}$

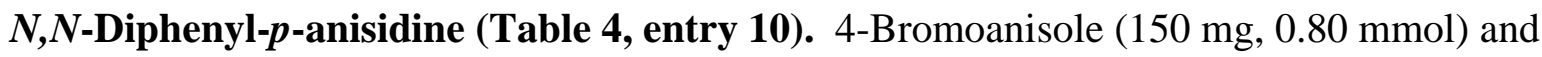
diphenylamine (132 mg, $0.78 \mathrm{mmol})$ were coupled using $\mathrm{Pd}_{2}(\mathrm{dba})_{3}(3.7 \mathrm{mg}, 4.0 \mu \mathrm{mol})$ and DTBNpP·HBF 4 (2.4 mg. $8 \mu \mathrm{mol})$ according to Method A. The crude product was purified by column chromatography (5\% ethyl acetate/95\% hexanes) to afford $N, N$-diphenyl-p-anisidine (179 mg, 83\%). ${ }^{1} \mathrm{H}$ NMR (500 MHz, $\left.\mathrm{CDCl}_{3}\right): 7.23$ (m, 4H), 7.07 (m, 6H), 6.97 (t, $J=7.22 \mathrm{~Hz}$, 2H), 6.86 (d, $J=9.12 \mathrm{~Hz}, 2 \mathrm{H}), 3.83(\mathrm{~s}, 3 \mathrm{H}) .{ }^{13} \mathrm{C} \mathrm{NMR}\left(90.6 \mathrm{MHz}, \mathrm{CDCl}_{3}\right): \delta 156.2,148.2$, 140.8, 129.1, 127.3, 122.9, 121.8, 114.8, 55.5. The NMR spectral data was identical to literature values. ${ }^{10} \mathrm{mp}: 97-99^{\circ} \mathrm{C}$ (lit. mp: $\left.98-100{ }^{\circ} \mathrm{C}\right) .{ }^{17}$

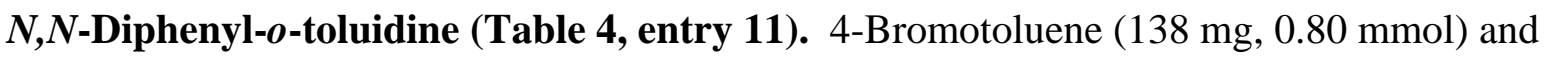
diphenylamine (132 mg, $0.78 \mathrm{mmol}$ ) were coupled using $\mathrm{Pd}_{2}(\mathrm{dba})_{3}(3.7 \mathrm{mg}, 4.0 \mu \mathrm{mol})$ and DTBNpP·HBF 4 (2.4 mg. $8 \mu \mathrm{mol})$ according to Method A. The crude product was purified by column chromatography (2.5\% ethyl acetate/97.5\% hexanes) and then by recrystallization from hot ethanol to afford 109 mg of $N, N$-diphenyl-o-toluidine contaminated with diphenyl amine 
(18\% by weight). The yield of $N, N$-diphenyl-o-toluidine was estimated to be $89.4 \mathrm{mg}(44 \%) .{ }^{1} \mathrm{H}$ NMR (500 MHz, CDCl ): $\delta 7.23$ (m, 9H), 6.99 (d, $J=8.54 \mathrm{~Hz}, 3 \mathrm{H}), 6.95(\mathrm{t}, J=7.28 \mathrm{~Hz}, 2 \mathrm{H})$, 2.07 (s, 3H). ${ }^{13} \mathrm{C}$ NMR $\left(90.6 \mathrm{MHz}, \mathrm{CDCl}_{3}\right): \delta 147.5,145.4,136.5,131.7,129.6,129.0,127.4$, 126.0, 121.5, 121.4, 18.6. The NMR spectral data was identical to literature values. ${ }^{10} \mathrm{mp}: 50-53$ ${ }^{\circ} \mathrm{C}$ (lit. mp: $\left.55-56{ }^{\circ} \mathrm{C}\right) .{ }^{17}$

Typical procedure for the coupling of aryl bromides and secondary alkylamines (Method A). $\mathrm{Pd}(\mathrm{OAc})_{2}(10 \mu \mathrm{mol}), \mathrm{DTBNpP}(10 \%$ solution in toluene) or DTBNpP·HBF 4 . $(10 \mu \mathrm{mol}, 1$ eq/Pd), sodium $t$-butoxide $(1.5 \mathrm{mmol})$, and toluene $(2.5 \mathrm{~mL})$ were added to a round bottom flask in a glove box. Upon removing the septum sealed flask from the drybox, the aryl bromide (1.0 mmol) and amine 12a or 12b $(1.0 \mathrm{mmol})$ were added by syringe and the reaction was allowed to stir for 2 hours at $50^{\circ} \mathrm{C}$. The reaction mixture was then adsorbed on silica gel and purified by flash chromatography.

\section{Typical procedure for the coupling of aryl chlorides and secondary alkylamines (Method}

B). $\mathrm{Pd}(\mathrm{OAc})_{2}(10-50 \mu \mathrm{mol}), \mathrm{DTBNpP}(10 \%$ solution in toluene) or DTBNpP·HBF $4 .(10-50$ $\mu \mathrm{mol}, 1 \mathrm{eq} / \mathrm{Pd})$, sodium $t$-butoxide $(1.5 \mathrm{mmol})$, and toluene $(2.5 \mathrm{~mL})$ were added to a round bottom flask in a glove box. Upon removing the septum sealed flask from the drybox, the aryl chloride (1.0 mmol) and amine 12a or $\mathbf{1 2 b}(1.0 \mathrm{mmol})$ were added by syringe. The flask was placed in a preheated oil bath at $80{ }^{\circ} \mathrm{C}$ and stirred until judged complete by GC. The reaction mixture was then adsorbed on silica gel and purified by flash chromatography.

$N$-(4-Trifluoromethylphenyl)morpholine (Table 5, entry 1). 4-Bromobenzotrifluoride (113 mg, $0.5 \mathrm{mmol}$ ) and morpholine (43 mg, $0.5 \mathrm{mmol}$ ) were coupled using $\operatorname{Pd}(\mathrm{OAc})_{2}$ (1.1 mg, 5 $\mu \mathrm{mol})$ and $\mathrm{DTBNpP} \cdot \mathrm{HBF}_{4}(1.5 \mathrm{mg} .5 \mu \mathrm{mol})$ according to Method A. The crude product was purified by column chromatography (5\% ethyl acetate/95\% hexanes) to afford $N$-(4- 
trifluoromethylphenyl)morpholine (108 mg, 93\%) as a white solid. ${ }^{1} \mathrm{H}$ NMR (360 MHz, DMSO): $\delta 7.51$ (d, $J=8.48 \mathrm{~Hz}, 2 \mathrm{H}), 7.05(\mathrm{~d}, J=8.74 \mathrm{~Hz}, 2 \mathrm{H}), 3.74(\mathrm{t}, J=4.87 \mathrm{~Hz}, 4 \mathrm{H}), 3.22$ (t, $J=4.87 \mathrm{~Hz}, 4 \mathrm{H}) .{ }^{13} \mathrm{C}$ NMR (90.6 MHz, DMSO): $\delta$ 153.3, $126.0\left(\mathrm{q}, J_{\mathrm{C}-\mathrm{F}}=3.68 \mathrm{~Hz}\right), 124.9$ $\left(\mathrm{q}, J_{\mathrm{C}-\mathrm{F}}=270.3 \mathrm{~Hz}\right), 118.2\left(\mathrm{q}, J_{\mathrm{C}-\mathrm{F}}=31.7 \mathrm{~Hz}\right), 113.9,65.8,47.1$. The ${ }^{1} \mathrm{H}$ NMR spectral data was identical to literature values. ${ }^{18} \mathrm{mp}: 64-66{ }^{\circ} \mathrm{C}$ (lit. mp: $58^{\circ} \mathrm{C}$ ). ${ }^{18}$

$N$-(4-Methylphenyl)morpholine (Table 5, entry 2; Table 6, entry 8). 4-Bromotoluene (123 $\mu \mathrm{L}, 1.00 \mathrm{mmol}$ ) and morpholine (87 $\mu \mathrm{L}, 1.0 \mathrm{mmol})$ were coupled using $\mathrm{Pd}(\mathrm{OAc})_{2}(2.2 \mathrm{mg}$, $10 \mu \mathrm{mol})$ and DTBNpP$\cdot \mathrm{HBF}_{4}(3.0 \mathrm{mg} .10 \mu \mathrm{mol})$ according to Method A. The crude product was purified by column chromatography (5\% ethyl acetate/95\% hexanes) to afford $N$-(4methylphenyl)morpholine (158 mg, 89\%) as a white solid. ${ }^{1} \mathrm{H}$ NMR (360 MHz, $\left.\mathrm{CDCl}_{3}\right): \delta 7.09$ (d, $J=8.24 \mathrm{~Hz}, 2 \mathrm{H}), 6.83$ (d, $J=8.58 \mathrm{~Hz}, 2 \mathrm{H}), 3.85$ (t, $J=4.80 \mathrm{~Hz}, 4 \mathrm{H}), 3.10$ (t, $J=4.80 \mathrm{~Hz}$, 4H), 2.27 (s, 3H). ${ }^{13} \mathrm{C}$ NMR (90.6 MHz, $\left.\mathrm{CDCl}_{3}\right): \delta 149.3,129.8,129.7,116.2,67.1,50.1,20.5$. The NMR spectral data was identical to literature values. ${ }^{10} \mathrm{mp}: 45-48{ }^{\circ} \mathrm{C}$ (lit. mp: $49.8-50.4$ $\left.{ }^{\circ} \mathrm{C}\right) .{ }^{19}$

Alternatively, 4-chlorotoluene (118 $\mu \mathrm{L}, 1.00 \mathrm{mmol}$ ) and morpholine ( $87 \mu \mathrm{L}, 1.0 \mathrm{mmol}$ ) were coupled using $\mathrm{Pd}(\mathrm{OAc})_{2}(4.4 \mathrm{mg}, 20 \mu \mathrm{mol})$ and DTBNpP·HBF $4(6.0 \mathrm{mg} .20 \mu \mathrm{mol})$ according to Method B to give $153 \mathrm{mg}$ of $\mathrm{N}$-(4-methylphenyl)morpholine (86\%).

$N$-(4-Methoxyphenyl)morpholine (Table 5, entry 3; Table 6, entry 9). 4-Bromoanisole (125 $\mu \mathrm{L}, 1.00 \mathrm{mmol}$ ) and morpholine (87 $\mu \mathrm{L}, 1.0 \mathrm{mmol})$ were coupled using $\mathrm{Pd}(\mathrm{OAc})_{2}(2.2 \mathrm{mg}$, $10 \mu \mathrm{mol})$ and DTBNpP·HBF 4 (3.0 mg. $10 \mu \mathrm{mol}$ ) according to Method A. The crude product was purified by column chromatography (10\% ethyl acetate/90\% hexanes) to afford $\mathrm{N}$-(4methoxyphenyl)morpholine (187 mg, 97\%) as a white solid. ${ }^{1} \mathrm{H}$ NMR (360 $\left.\mathrm{MHz}, \mathrm{CDCl}_{3}\right): \delta$ 6.89 (d, $J=9.40 \mathrm{~Hz}, 2 \mathrm{H}), 6.85$ (d, $J=9.40 \mathrm{~Hz}, 2 \mathrm{H}$ ), 3.85 (t, $J=4.75 \mathrm{~Hz}, 4 \mathrm{H}$ ), 3.77 (s, 3H), 3.05 
(t, $J=4.75 \mathrm{~Hz}, 4 \mathrm{H}) .{ }^{13} \mathrm{C} \mathrm{NMR}\left(90.6 \mathrm{MHz}, \mathrm{CDCl}_{3}\right): \delta 154.0,145.7,117.9,114.5,67.1,55.6$,

50.9. The NMR spectral data was identical to literature values. ${ }^{10} \mathrm{mp}: 69-71^{\circ} \mathrm{C}$ (lit. mp: 71 $\left.{ }^{\circ} \mathrm{C}\right) .{ }^{19}$

Alternatively, 4-chloroanisole (122 $\mu \mathrm{L}, 1.00 \mathrm{mmol}$ ) and morpholine (87 $\mu \mathrm{L}, 1.0 \mathrm{mmol})$ were coupled using $\mathrm{Pd}(\mathrm{OAc})_{2}(11.2 \mathrm{mg}, 50 \mu \mathrm{mol})$ and $\mathrm{DTBNpP} \cdot \mathrm{HBF}_{4}(15 \mathrm{mg} .50 \mu \mathrm{mol})$ according to Method B to give $153 \mathrm{mg}$ of $\mathrm{N}$-(4-methoxyphenyl)morpholine (79\%).

$N$-(4-( $N^{\prime}, N^{\prime}$-Dimethylamino)phenyl)morpholine (Table 5, entry 4). 4-Bromo- $N, N-$ dimethylaniline (200 g, $1.00 \mathrm{mmol})$ and morpholine (87 $\mu \mathrm{L}, 1.0 \mathrm{mmol})$ were coupled using $\mathrm{Pd}(\mathrm{OAc})_{2}(2.2 \mathrm{mg}, 10 \mu \mathrm{mol})$ and $\mathrm{DTBNpP} \cdot \mathrm{HBF}_{4}(3.0 \mathrm{mg} .10 \mu \mathrm{mol})$ according to Method A. The crude product was purified by column chromatography (10\% ethyl acetate/90\% methylene chloride) to afford $N$-(4-methoxyphenyl)morpholine (187 mg, 97\%) as an off-white solid. ${ }^{1} \mathrm{H}$ NMR (360 MHz, CDCl $)$ ): $\delta 6.89$ (d, $J=9.08 \mathrm{~Hz}, 2$ H), 6.75 (d, $J=9.08 \mathrm{~Hz}, 2 \mathrm{H}), 3.85$ (t, $J=$ $4.75 \mathrm{~Hz}, 4 \mathrm{H}), 3.03(\mathrm{t}, J=4.75 \mathrm{~Hz}, 4 \mathrm{H}), 2.87(\mathrm{~s}, 3 \mathrm{H}) .{ }^{13} \mathrm{C} \mathrm{NMR}\left(90.6 \mathrm{MHz}, \mathrm{CDCl}_{3}\right): \delta 145.9$, 143.3, 118.1, 114.6, 67.2, 51.2, 41.6. The NMR spectral data was identical to literature values. ${ }^{20}$ mp: $117-120^{\circ} \mathrm{C}$.

$N$-(2-Methylphenyl)morpholine (Table 5, entry 5). 4-Bromotoluene (120 $\mu \mathrm{L}, 1.00 \mathrm{mmol})$ and morpholine (87 $\mu \mathrm{L}, 1.0 \mathrm{mmol}$ ) were coupled using $\mathrm{Pd}(\mathrm{OAc})_{2}(2.2 \mathrm{mg}, 10 \mu \mathrm{mol})$ and DTBNpP·HBF 4 (3.0 mg. $10 \mu \mathrm{mol}$ ) according to Method A. The crude product was purified by column chromatography (5\% ethyl acetate/95\% hexanes) to afford $N$-(2methylphenyl)morpholine (165 mg, 93\%) as a colorless oil. ${ }^{1} \mathrm{H}$ NMR (360 MHz, $\left.\mathrm{CDCl}_{3}\right): \delta$ 7.20-7.16 (m, 2H), 7.02-6.97 (m, 2H), 3.85 (t, $J=4.52 \mathrm{~Hz}, 4 \mathrm{H}), 2.90$ (t, $J=4.52 \mathrm{~Hz}, 4 \mathrm{H}), 2.32$ (s, 3H). ${ }^{13} \mathrm{C}$ NMR $\left(90.6 \mathrm{MHz}, \mathrm{CDCl}_{3}\right): \delta 151.4,132.7,131.3,126.8,123.5,119.1,67.6,52.4$, 18.0. The NMR spectral data was identical to literature values. ${ }^{14}$ 
$N$-(2,4-Dimethylphenyl)morpholine (Table 5, entry 6). 4-Bromo-m-xylene (135 $\mu \mathrm{L}, 1.00$ mmol) and morpholine (87 $\mu \mathrm{L}, 1.0 \mathrm{mmol})$ were coupled using $\mathrm{Pd}(\mathrm{OAc})_{2}(2.2 \mathrm{mg}, 10 \mu \mathrm{mol})$ and DTBNpP·HBF 4 (3.0 mg. $10 \mu \mathrm{mol})$ according to Method A. The crude product was purified by column chromatography (5\% ethyl acetate/95\% hexanes) to afford $\mathrm{N}$-(2,4dimethylphenyl)morpholine (168 mg, 88\%) as a colorless oil. ${ }^{1} \mathrm{H}$ NMR (360 MHz, $\left.\mathrm{CDCl}_{3}\right): \delta$ 7.01-6.97 (m, 2H), 6.93-6.91 (m 1H), $3.83(\mathrm{t}, J=4.55 \mathrm{~Hz}, 4 \mathrm{H}), 2.89$ (t, $J=4.55 \mathrm{~Hz}, 4 \mathrm{H}), 2.28$ (s, 3H), 2.27 (s, 3H). ${ }^{13} \mathrm{C}$ NMR (90.6 MHz, $\left.\mathrm{CDCl}_{3}\right): \delta 148.9,132.9,132.7,132.0,127.2,119.0$, 67.6, 52.6, 20.8, 17.8 .

$N, N$-Dibutyl-p-toluidine (Table 5, entry 6; Table 6, entry 10). 4-Bromotoluene (123 $\mu \mathrm{L}$, $1.00 \mathrm{mmol})$ and dibutylamine $(169 \mu \mathrm{L}, 1.0 \mathrm{mmol})$ were coupled using $\mathrm{Pd}(\mathrm{OAc})_{2}(2.2 \mathrm{mg}, 10$ $\mu \mathrm{mol})$ and DTBNpP·HBF 4 (3.0 mg. $10 \mu \mathrm{mol})$ according to Method A. The crude product was purified by column chromatography (2.5\% ethyl acetate/97.5\% hexanes) to afford $N, N$-dibutyl-ptoluidine (168 mg, 88\%) as a pale yellow oil. ${ }^{1} \mathrm{H}$ NMR (360 MHz, $\left.\mathrm{CDCl}_{3}\right): \delta 7.01(\mathrm{~d}, J=8.26$ Hz, 2H), 6.57 (d, $J=8.69 \mathrm{~Hz}, 2 \mathrm{H}), 3.21$ (t, $J=7.60 \mathrm{~Hz}, 4 \mathrm{H}), 2.37$ (s, 3H), 1.58-1.49 (m, 4H), 1.38-1.28 (m, 4H), $0.93(\mathrm{t}, J=7.32 \mathrm{~Hz}, 6 \mathrm{H}) .{ }^{13} \mathrm{C} \mathrm{NMR}\left(90.6 \mathrm{MHz}, \mathrm{CDCl}_{3}\right): \delta 146.3,129.8$, 124.4, 112.3, 51.1, 29.6, 20.5, 20.3 14.1. The NMR spectral data was identical to literature values. $^{19}$

Alternatively, 4-chlorotoluene (118 $\mu \mathrm{L}, 1.00 \mathrm{mmol})$ and dibutylamine (169 $\mu \mathrm{L}, 1.0 \mathrm{mmol})$ were coupled using Pd(OAc) 2 (4.4 mg, $20 \mu \mathrm{mol})$ and DTBNpP·HBF 4 (6.1 mg. $20 \mu \mathrm{mol})$ according to Method B to give $216 \mathrm{mg}$ of $N, N$-dibutyl-p-toluidine (98\%).

$N, N$-Dibutyl-p-anisidine (Table 5, entry 7). 4-Bromoanisole (125 $\mu \mathrm{L}, 1.00 \mathrm{mmol}$ ) and dibutylamine (169 $\mu \mathrm{L}, 1.0 \mathrm{mmol})$ were coupled using $\mathrm{Pd}(\mathrm{OAc})_{2}(2.2 \mathrm{mg}, 10 \mu \mathrm{mol})$ and DTBNpP·HBF 4 (3.0 mg. $10 \mu \mathrm{mol}$ ) according to Method A. The crude product was purified by 
column chromatography ( $2.5 \%$ ethyl acetate/97.5\% hexanes) to afford $N, N$-dibutyl-p-anisidine (168 mg, 88\%) as a pale yellow oil. ${ }^{1} \mathrm{H}$ NMR (360 MHz, $\left.\mathrm{CDCl}_{3}\right): \delta 6.81$ (d, $\left.J=9.12 \mathrm{~Hz}, 2 \mathrm{H}\right)$, 6.64 (d, $J=9.12 \mathrm{~Hz}), 3.74$ (s, 3H), 3.17 (t, $J=7.60 \mathrm{~Hz}, 4 \mathrm{H}), 1.56-1.47$ (m, 4H), 1.34-1.28 (m, 4H), 0.93 (t, $J=7.32 \mathrm{~Hz}, 6 \mathrm{H}) .{ }^{13} \mathrm{C} \mathrm{NMR}\left(90.6 \mathrm{MHz}, \mathrm{CDCl}_{3}\right): \delta 151.1,143.4,114.9,114.5$, 55.9, 51.8, 29.6, 20.6, 14.2. The NMR spectral data was identical to literature values. ${ }^{21}$

Alternatively, 4-chlorotoluene (118 $\mu \mathrm{L}, 1.00 \mathrm{mmol})$ and dibutylamine (169 $\mu \mathrm{L}, 1.0 \mathrm{mmol})$ were coupled using $\mathrm{Pd}(\mathrm{OAc})_{2}(11 \mathrm{mg}, 50 \mu \mathrm{mol})$ and DTBNpP·HBF $4(15 \mathrm{mg} .50 \mu \mathrm{mol})$ according to Method B to give $206 \mathrm{mg}$ of $N, N$-dibutyl-p-anisidine (86\%).

$N$-(4-Cyanophenyl)morpholine (Table 6, entry 7). 4-Chlorobenzonitrile (138 mg, 1.00 mmol) and morpholine (87 $\mu \mathrm{L}, 1.0 \mathrm{mmol}$ ) were coupled using $\mathrm{Pd}(\mathrm{OAc})_{2}(2.2 \mathrm{mg}, 10 \mu \mathrm{mol})$ and DTBNpP·HBF 4 (3.0 mg. $10 \mu \mathrm{mol}$ ) according to Method B at $80^{\circ} \mathrm{C}$. The crude product was purified by column chromatography (20\% ethyl acetate/80\% hexanes) to afford $N$-(4cyanophenyl)morpholine (132 mg, 70\%) as a pale yellow solid. ${ }^{1} \mathrm{H}$ NMR (360 MHz, $\left.\mathrm{CDCl}_{3}\right): \delta$ 7.52 (d, $J=9.05 \mathrm{~Hz}, 2 \mathrm{H}), 6.86$ (d, $J=9.05 \mathrm{~Hz}, 2 \mathrm{H}), 3.85(\mathrm{t}, J=4.94 \mathrm{~Hz}, 4 \mathrm{H}), 3.28$ (t, $J=4.94$ $\mathrm{Hz}, 4 \mathrm{H}) .{ }^{13} \mathrm{C}$ NMR $\left(90.6 \mathrm{MHz}, \mathrm{CDCl}_{3}\right): \delta 153.6,133.6,119.9,114.2,101.1,66.6,47.4$. The NMR spectral data was identical to literature values. ${ }^{22} \mathrm{mp}: 77-81^{\circ} \mathrm{C}$ (lit. mp: $77-78{ }^{\circ} \mathrm{C}$ ) ${ }^{22}$

Structure optimization and determination of cone angles. Geometries were optimized at the LDFT level with the polarized double zeta DZVP2 basis set on all atoms except Pd. ${ }^{23}$ We used the Stuttgart relativistic pseudopotential and the associated basis set for Pd. ${ }^{24}$ There are 28 electrons in the core of the ECP and the basis set for Pd is contracted to [6s5p3d]. The LDFT calculations were done with the potential fit of Vosko, Wilk and Nusair for the correlation functional ${ }^{25}$ and the exchange functional of Slater. ${ }^{26}$ The bond energies were calculated at the gradient-corrected level at the LDA optimized geometries with the B3LYP exchange-correlation 
functional with the above basis sets. ${ }^{27,28}$ The calculations were done with the program Gaussian03 ${ }^{29}$ on Cray XD-1 and Silicon Graphics Altix computers at the Alabama

Supercomputing Center. The cone angles were calculated from the LDA optimized geometries for the (phosphine) $\mathrm{Pd}^{0}$ complex by using the STERIC program and the volumetric parameters for the atoms therein with the Pd atom set at the origin. .0,31 $^{3}$

\section{References.}

(1) Ohwada, A.; Nara, S.; Sakamoto, T.; Kikugawa, Y. J. Chem. Soc., Perkin Trans. 1 2001, 3064-3068.

(2) Yu, Y.; Srogl, J.; Liebeskind, L. S. Org. Lett. 2004, 6, 2361-2634.

(3) Kozachuk, D. N.; Serguchev, Y. A.; Kremlev, M. M.; Fialkov, Y. A.; Yagupol'skii, L. M. Zh. Obsh. Khim. 1974, 1230.

(4) Li, G. Y.; Zeng, G.; Noonan, A. F. J. Org. Chem. 2001, 66, 8677-8681.

(5) McNab, H. J. Chem. Soc., Perkin Trans. 1 1980, 2200-2204.

(6) Gajare, A. S.; Toyota, K.; Yioshifuji, M.; Ozawa, F. J. Org. Chem. 2004, 69, 6504-6506.

(7) Zhang, H.; Cai, Q.; Ma, D. J. Org. Chem. 2005, 70, 5164-5173.

(8) Neugebauer, F. A.; Fischer, H. Chem. Ber. 1971, 104, 886.

(9) Hamann, B. C.; Hartwig, J. F. J. Am. Chem. Soc. 1998, 120, 3694-3703.

(10) Kuwano, R.; Utsunomiya, M.; Hartwig, J. F. J. Org. Chem. 2002, 67, 6479-6486.

(11) Sunberg, R. J.; Sloan, K. B. J. Org. Chem. 1973, 38, 2052-2057.

(12) Grasa, G. A.; Viciu, M. S.; Huang, J.; Nolan, S. P. J. Org. Chem. 2001, 66, 7729-7737.

(13) Freeman, H. S.; Butler, J. R.; Freedman, L. D. J. Org. Chem. 1978, 43, 4975-4978.

(14) Hartwig, J. F.; Kawatsura, M.; Hauck, S. I.; Shaughnessy, K. H.; Alcazar-Roman, L. M. J. Org. Chem. 1999, 64, 5575-5580.

(15) Rataboul, F.; Zapf, A.; Jackstell, R.; Harkal, S.; Reiermeier, T.; Monsees, A.; Dingerdissen, U.; Beller, M. Chem.--Eur. J. 2004, 10, 2983-2990.

(16) Ciminale, F.; Ciardo, A.; Francioso, S.; Nacci, A. J. Org. Chem. 1999, 64, 2459-2464.

(17) Chen, C.; Yang, L.-M. Org. Lett. 2005, 7, 2209-2211. 
(18) Kamikawa, K.; Sugimoto, S.; Uemura, M. J. Org. Chem. 1998, 63, 8407-8410.

(19) Wolfe, J. P.; Buchwald, S. L. J. Org. Chem. 1996, 61, 133-1135.

(20) Gerristma, D.; Brenstrum, T.; McNulty, J.; Capretta, A. Tetrahedron Lett. 2004, 45, 8319-8321.

(21) Marcoux, J.-F.; Wagaw, S.; Buchwald, S. L. J. Org. Chem. 1997, 62, 1568-1569.

(22) Wolfe, J. P.; Buchwald, S. L. J. Am. Chem. Soc. 1997, 119, 6054-6058.

(23) Godbout, N.; Salahub, D. R.; Andzelm, J.; Wimmer, E. Can. J. Chem. 1992, 70, 560-571.

(24) Küchle, W.; Dolg, M.; Stoll, H.; Preuss, H. J. Chem. Phys. 1994, 100, 7535-7542 and references therein; http://www.theochem.uni-stuttgart.de/pseudopotentials/.

(25) Vosko, S. J.; Wilk, L.; Nusair, W. Can. J. Phys. 1980, 58, 1200.

(26) Slater, J. C. Phys. Rev. 1951, 81, 385.

(27) Becke, A. D. J. Chem. Phys. 1993, 98, 5648-5662.

(28) Lee, C.; Yang, W.; Parry, R. G. Phys. Rev. B 1988, 37, 785-789.

(29) Frisch, M. J., et al, Gaussian 03, Revision C.02, Gaussian Inc, 2004.

(30) Taverner, B. C. J. Comput. Chem. 1996, 17, 1612-1623.

(31) Taverner, B. C., STERIC, 1.11, http://www.ccl.net/cca/software/SOURCES/C/steric/index.shtml, 1995. 


\section{Computational Data.}

Pd, L and (L)Pd Total Electronic Energies and Zero Point Energies (ZPE) in a.u. Species $\quad$ E(B3LYP/DGDZVP2) ZPE(SVWN5/DGDZVP2)

$\begin{array}{ccc}\mathrm{Pd} & -127.886371 & 0.000000 \\ \text { TTBP } & -814.998386 & 0.356952 \\ \mathrm{DTBNpP} & -854.338499 & 0.382832 \\ \text { TBDNpP } & -893.671314 & 0.409430 \\ \mathrm{TnpP} & -933.000191 & 0.437614 \\ (\mathrm{i}-\mathrm{Pr})_{3} \mathrm{P} & -697.056917 & 0.275630 \\ (\mathrm{n}-\mathrm{Bu})_{3} \mathrm{P} & -815.041528 & 0.358265 \\ \mathrm{Ph}{ }_{3} \mathrm{P} & -1036.423898 & 0.266275 \\ & & \\ \mathrm{Pd}(\mathrm{TTBP}) & -942.944770 & 0.357467 \\ \mathrm{Pd}(\mathrm{DTBNp}) & -982.282697 & 0.383567 \\ \mathrm{Pd}(\mathrm{TBDNp}) & -1021.613819 & 0.409441 \\ \mathrm{Pd}(\mathrm{TNpP}) & -1060.940079 & 0.276806 \\ \left.\mathrm{Pd}(\mathrm{i}-\mathrm{Pr})_{3} \mathrm{P}\right] & -825.006197 & 0.359467 \\ \mathrm{Pd}\left[(\mathrm{n}-\mathrm{Bu})_{3} \mathrm{P}\right] & -942.991629 & 0.267315 \\ \mathrm{Pd}\left(\mathrm{Ph}{ }_{3} \mathrm{P}\right) & -1164.370427 & \end{array}$

The local DFT optimized geometries (coordinates) of TTBP, DTBNpP, TBDNpP, TNpP, (TTBP)Pd, (DTBNpP), (TBDNpP)Pd, and (TNpP)Pd in $x, y, z$ format in $\AA$.

\begin{tabular}{lrrr}
\multicolumn{4}{l}{ TTBP } \\
P & 0.000000 & 0.000000 & 0.731735 \\
C & 0.000000 & 1.760795 & 0.005384 \\
C & 0.387261 & 1.923952 & -1.457653 \\
C & -1.381020 & 2.373965 & 0.208669 \\
C & 0.944757 & 2.590608 & 0.875305 \\
C & 1.524893 & -0.880398 & 0.005384 \\
C & 1.472560 & -1.297354 & -1.457653 \\
C & 2.746424 & 0.009016 & 0.208669 \\
C & 1.771154 & -2.113488 & 0.875305 \\
C & -1.524893 & -0.880398 & 0.005384 \\
C & -1.859822 & -0.626598 & -1.457653 \\
C & -1.365404 & -2.382981 & 0.208669 \\
C & -2.715911 & -0.477120 & 0.875305 \\
H & 0.664235 & 2.515749 & 1.941537 \\
H & 0.870211 & 3.654450 & 0.572857 \\
H & 2.000154 & 2.290041 & 0.782846 \\
H & -1.729228 & 2.262393 & 1.252426
\end{tabular}




$\begin{array}{rrrr}\mathrm{H} & -2.144951 & 1.948780 & -0.464901 \\ \mathrm{H} & -1.317371 & 3.459086 & -0.007709 \\ \mathrm{H} & 1.426858 & 1.610178 & -1.657075 \\ \mathrm{H} & 0.313657 & 2.995406 & -1.735203 \\ \mathrm{H} & -0.276644 & 1.358039 & -2.133677 \\ \mathrm{H} & 1.846585 & -1.833119 & 1.941537 \\ \mathrm{H} & 2.729741 & -2.580850 & 0.572857 \\ \mathrm{H} & 0.983157 & -2.877205 & 0.782846 \\ \mathrm{H} & 2.823904 & 0.366359 & 1.252426 \\ \mathrm{H} & 2.760168 & 0.883192 & -0.464901 \\ \mathrm{H} & 3.654341 & -0.588666 & -0.007709 \\ \mathrm{H} & 0.681026 & -2.040785 & -1.657075 \\ \mathrm{H} & 2.437269 & -1.769338 & -1.735203 \\ \mathrm{H} & 1.314418 & -0.439438 & -2.133677 \\ \mathrm{H} & -2.510820 & -0.682630 & 1.941537 \\ \mathrm{H} & -3.599952 & -1.073600 & 0.572857 \\ \mathrm{H} & -2.983311 & 0.587164 & 0.782846 \\ \mathrm{H} & -1.094676 & -2.628752 & 1.252426 \\ \mathrm{H} & -0.615217 & -2.831972 & -0.464901 \\ \mathrm{H} & -2.336971 & -2.870419 & -0.007709 \\ \mathrm{H} & -2.107884 & 0.430606 & -1.657075 \\ \mathrm{H} & -2.750926 & -1.226068 & -1.735203 \\ \mathrm{H} & -1.037774 & -0.918601 & -2.133677\end{array}$

\section{DTBNpP}

$\begin{array}{lrrr}\mathrm{P} & -0.330400 & 0.000687 & -0.532911 \\ \mathrm{C} & -1.272577 & 1.543395 & 0.026447 \\ \mathrm{C} & -1.868078 & 1.501063 & 1.422468 \\ \mathrm{C} & -0.283387 & 2.698912 & -0.051759 \\ \mathrm{C} & -2.366670 & 1.816123 & -0.998703 \\ \mathrm{C} & -1.312623 & -1.523315 & 0.014213 \\ \mathrm{C} & -1.444155 & -1.775852 & 1.508556 \\ \mathrm{C} & -2.699370 & -1.472288 & -0.608324 \\ \mathrm{C} & -0.553747 & -2.692470 & -0.603251 \\ \mathrm{C} & 2.489957 & -0.013560 & 0.019851 \\ \mathrm{C} & 2.687849 & -1.230749 & -0.870263 \\ \mathrm{C} & 2.675035 & 1.241631 & -0.817833 \\ \mathrm{C} & 3.525379 & -0.030990 & 1.134083 \\ \mathrm{H} & -1.946176 & 1.875872 & -2.019102 \\ \mathrm{H} & -2.855544 & 2.784482 & -0.771778 \\ \mathrm{H} & -3.148040 & 1.037730 & -1.000705 \\ \mathrm{H} & 0.201961 & 2.756401 & -1.043792 \\ \mathrm{H} & 0.513085 & 2.627327 & 0.711024 \\ \mathrm{H} & -0.825427 & 3.649873 & 0.119005 \\ \mathrm{H} & -2.711684 & 0.790707 & 1.489357 \\ \mathrm{H} & -2.261051 & 2.502979 & 1.689953\end{array}$




$\begin{array}{lrrr}\mathrm{H} & -1.119248 & 1.223560 & 2.188421 \\ \mathrm{H} & -0.477722 & -2.590544 & -1.701412 \\ \mathrm{H} & -1.081510 & -3.639939 & -0.375365 \\ \mathrm{H} & 0.473799 & -2.775311 & -0.201559 \\ \mathrm{H} & -2.656508 & -1.205321 & -1.681035 \\ \mathrm{H} & -3.357147 & -0.746573 & -0.095735 \\ \mathrm{H} & -3.179313 & -2.467630 & -0.524491 \\ \mathrm{H} & -0.463225 & -1.893040 & 2.004412 \\ \mathrm{H} & -1.997256 & -2.723308 & 1.667835 \\ \mathrm{H} & -1.993270 & -0.974573 & 2.029143 \\ \mathrm{H} & 3.419846 & 0.853336 & 1.790530 \\ \mathrm{H} & 4.550910 & -0.023888 & 0.719502 \\ \mathrm{H} & 3.420674 & -0.935844 & 1.762161 \\ \mathrm{H} & 2.576155 & 2.156642 & -0.204255 \\ \mathrm{H} & 1.918999 & 1.284673 & -1.625452 \\ \mathrm{H} & 3.678074 & 1.249005 & -1.283581 \\ \mathrm{H} & 2.631610 & -2.168772 & -0.284548 \\ \mathrm{H} & 3.678135 & -1.196628 & -1.361219 \\ \mathrm{H} & 1.911227 & -1.268606 & -1.658673 \\ \mathrm{C} & 1.095338 & -0.057069 & 0.660274 \\ \mathrm{H} & 0.996859 & 0.771193 & 1.391500 \\ \mathrm{H} & 1.020079 & -0.993098 & 1.248958\end{array}$

\section{TBDNpP}

$\begin{array}{lrrr}\text { P } & 0.027790 & 0.404776 & -0.387793 \\ \mathrm{C} & 2.538032 & -0.935981 & 0.023181 \\ \mathrm{C} & 1.823963 & -2.277308 & -0.008585 \\ \mathrm{C} & 2.994185 & -0.571980 & -1.381721 \\ \mathrm{C} & 3.748342 & -1.032771 & 0.940018 \\ \mathrm{C} & -0.264936 & 2.218935 & 0.036556 \\ \mathrm{C} & -0.086803 & 2.545632 & 1.509632 \\ \mathrm{C} & 0.724049 & 3.020095 & -0.797935 \\ \mathrm{C} & -1.673549 & 2.594596 & -0.389234 \\ \mathrm{C} & -2.279248 & -1.291308 & 0.023551 \\ \mathrm{C} & -3.137141 & -0.409986 & -0.870240 \\ \mathrm{C} & -1.665365 & -2.392509 & -0.827789 \\ \mathrm{C} & -3.155735 & -1.920916 & 1.096566 \\ \mathrm{H} & 4.288669 & -0.068667 & 0.991044 \\ \mathrm{H} & 4.457145 & -1.799671 & 0.575297 \\ \mathrm{H} & 3.448772 & -1.309065 & 1.968629 \\ \mathrm{H} & 3.566080 & 0.375745 & -1.378567 \\ \mathrm{H} & 2.123399 & -0.440972 & -2.051525 \\ \mathrm{H} & 3.644710 & -1.361609 & -1.801122 \\ \mathrm{H} & 1.460117 & -2.562375 & 0.998023 \\ \mathrm{H} & 2.504618 & -3.074600 & -0.360988 \\ \mathrm{H} & 0.952753 & -2.246810 & -0.689645\end{array}$




$\begin{array}{crrr}\mathrm{H} & -1.858736 & 2.350828 & -1.451882 \\ \mathrm{H} & -1.824046 & 3.683906 & -0.255740 \\ \mathrm{H} & -2.439534 & 2.076188 & 0.216730 \\ \mathrm{H} & 0.583185 & 2.825811 & -1.877013 \\ \mathrm{H} & 1.775496 & 2.783522 & -0.548218 \\ \mathrm{H} & 0.577603 & 4.103641 & -0.620411 \\ \mathrm{H} & -0.773083 & 1.959971 & 2.150318 \\ \mathrm{H} & -0.310483 & 3.616838 & 1.685276 \\ \mathrm{H} & 0.945230 & 2.362542 & 1.858413 \\ \mathrm{H} & -2.566185 & -2.590146 & 1.751211 \\ \mathrm{H} & -3.965627 & -2.520660 & 0.640261 \\ \mathrm{H} & -3.625074 & -1.147857 & 1.733713 \\ \mathrm{H} & -1.062258 & -3.086846 & -0.212689 \\ \mathrm{H} & -1.011387 & -1.960744 & -1.610006 \\ \mathrm{H} & -2.457296 & -2.979326 & -1.329650 \\ \mathrm{H} & -3.659969 & 0.372929 & -0.289681 \\ \mathrm{H} & -3.903365 & -1.017834 & -1.386152 \\ \mathrm{H} & -2.518384 & 0.088591 & -1.641250 \\ \mathrm{C} & -1.182932 & -0.476527 & 0.721002 \\ \mathrm{H} & -0.592499 & -1.161714 & 1.362271 \\ \mathrm{H} & -1.656185 & 0.260635 & 1.403230 \\ \mathrm{C} & 1.608481 & 0.151352 & 0.562591 \\ \mathrm{H} & 1.374194 & -0.046931 & 1.629473 \\ \mathrm{H} & 2.157235 & 1.115110 & 0.531976\end{array}$

\begin{tabular}{lrcc}
\multicolumn{4}{l}{ TNpP } \\
P & 0.000000 & 0.000000 & 0.095653 \\
C & 0.000000 & 2.852099 & -0.014138 \\
C & -1.474064 & 2.818018 & 0.361843 \\
C & 0.838836 & 2.947159 & 1.252166 \\
C & 0.271338 & 4.062350 & -0.893920 \\
C & 2.469990 & -1.426049 & -0.014138 \\
C & 3.382430 & -2.266161 & -0.893920 \\
C & 3.177507 & -0.132432 & 0.361843 \\
$\mathrm{C}$ & 2.132897 & -2.200033 & 1.252166 \\
$\mathrm{C}$ & -2.469990 & -1.426049 & -0.014138 \\
$\mathrm{C}$ & -1.703443 & -2.685586 & 0.361843 \\
$\mathrm{C}$ & -2.971733 & -0.747126 & 1.252166 \\
$\mathrm{C}$ & -3.653768 & -1.796189 & -0.893920 \\
$\mathrm{H}$ & 1.336182 & 4.106555 & -1.190111 \\
$\mathrm{H}$ & 0.028295 & 5.000793 & -0.361042 \\
$\mathrm{H}$ & -0.336557 & 4.027854 & -1.817536 \\
$\mathrm{H}$ & 1.916578 & 3.013165 & 1.008321 \\
$\mathrm{H}$ & 0.685463 & 2.054089 & 1.888137 \\
$\mathrm{H}$ & 0.566438 & 3.843915 & 1.839058 \\
$\mathrm{H}$ & -2.116775 & 2.750762 & -0.537357
\end{tabular}




$\begin{array}{lrrr}\mathrm{H} & -1.756924 & 3.734941 & 0.911419 \\ \mathrm{H} & -1.700288 & 1.949938 & 1.013107 \\ \mathrm{H} & 1.436162 & -1.620673 & 1.888137 \\ \mathrm{H} & 3.045709 & -2.412507 & 1.839058 \\ \mathrm{H} & 1.651188 & -3.166388 & 1.008321 \\ \mathrm{H} & 2.538840 & 0.497524 & 1.013107 \\ \mathrm{H} & 3.440617 & 0.457800 & -0.537357 \\ \mathrm{H} & 4.113015 & -0.345930 & 0.911419 \\ \mathrm{H} & 2.888290 & -3.210445 & -1.190111 \\ \mathrm{H} & 4.316666 & -2.524901 & -0.361042 \\ \mathrm{H} & 3.656502 & -1.722460 & -1.817536 \\ \mathrm{H} & -4.224472 & -0.896110 & -1.190111 \\ \mathrm{H} & -4.344961 & -2.475892 & -0.361042 \\ \mathrm{H} & -3.319946 & -2.305394 & -1.817536 \\ \mathrm{H} & -3.567766 & 0.153223 & 1.008321 \\ \mathrm{H} & -2.121625 & -0.433416 & 1.888137 \\ \mathrm{H} & -3.612146 & -1.431408 & 1.839058 \\ \mathrm{H} & -1.323842 & -3.208561 & -0.537357 \\ \mathrm{H} & -2.356092 & -3.389011 & 0.911419 \\ \mathrm{H} & -0.838552 & -2.447462 & 1.013107 \\ \mathrm{C} & -1.568767 & -0.460746 & -0.786611 \\ \mathrm{H} & -2.125172 & 0.480984 & -0.965992 \\ \mathrm{H} & -1.316720 & -0.883128 & -1.782311 \\ \mathrm{C} & 0.385366 & 1.588965 & -0.786611 \\ \mathrm{H} & -0.106451 & 1.581877 & -1.782311 \\ \mathrm{H} & 1.479130 & 1.599961 & -0.965992 \\ \mathrm{C} & 1.183402 & -1.128219 & -0.786611 \\ \mathrm{H} & 0.646042 & -2.080945 & -0.965992 \\ \mathrm{H} & 1.423171 & -0.698749 & -1.782311 \\ & & & \\ & & & \\ & & \end{array}$

\begin{tabular}{lrrr}
\multicolumn{4}{l}{$(\mathbf{i}-\mathbf{P r})_{3} \mathbf{P}$} \\
$\mathrm{P}$ & 0.033956 & 0.025746 & -0.697990 \\
$\mathrm{C}$ & 1.692815 & -0.077525 & 0.147891 \\
$\mathrm{C}$ & 2.350903 & -1.400713 & -0.213409 \\
$\mathrm{C}$ & 2.587305 & 1.059903 & -0.320620 \\
$\mathrm{C}$ & -0.951075 & 1.235857 & 0.343531 \\
$\mathrm{C}$ & -0.136046 & 2.377366 & 0.922865 \\
$\mathrm{C}$ & -2.076521 & 1.794226 & -0.515362 \\
$\mathrm{C}$ & -0.730494 & -1.578100 & -0.106756 \\
$\mathrm{C}$ & -0.594537 & -1.860918 & 1.379415 \\
$\mathrm{C}$ & -2.187202 & -1.648320 & -0.535317 \\
$\mathrm{H}$ & 2.723053 & 1.007716 & -1.417281 \\
$\mathrm{H}$ & 3.587436 & 0.983730 & 0.147396 \\
$\mathrm{H}$ & 2.178163 & 2.056104 & -0.084343 \\
$\mathrm{H}$ & 2.385307 & -1.527372 & -1.313395 \\
$\mathrm{H}$ & 1.828701 & -2.276738 & 0.210533
\end{tabular}




$\begin{array}{rrrc}\mathrm{H} & 3.395246 & -1.420877 & 0.151272 \\ \mathrm{H} & -1.658020 & 2.396100 & -1.343645 \\ \mathrm{H} & -2.740895 & 2.449011 & 0.080909 \\ \mathrm{H} & -2.695681 & 1.001510 & -0.971091 \\ \mathrm{H} & 0.306848 & 2.996745 & 0.119599 \\ \mathrm{H} & 0.680640 & 2.031394 & 1.581756 \\ \mathrm{H} & -0.791039 & 3.042587 & 1.517515 \\ \mathrm{H} & -2.312217 & -1.427512 & -1.611332 \\ \mathrm{H} & -2.802354 & -0.923647 & 0.032306 \\ \mathrm{H} & -2.606388 & -2.652671 & -0.338242 \\ \mathrm{H} & 0.456762 & -1.851116 & 1.720742 \\ \mathrm{H} & -1.020001 & -2.852815 & 1.624879 \\ \mathrm{H} & -1.149135 & -1.117562 & 1.983364 \\ \mathrm{H} & -1.387859 & 0.661079 & 1.187352 \\ \mathrm{H} & 1.553375 & -0.019921 & 1.248460 \\ \mathrm{H} & -0.172180 & -2.352584 & -0.670334\end{array}$

\begin{tabular}{lrcc}
\multicolumn{4}{l}{$\mathbf{( n - B u})_{3} \mathbf{P}$} \\
$\mathrm{P}$ & -0.020652 & 0.006604 & -0.756144 \\
$\mathrm{C}$ & 0.178120 & -1.622313 & 0.101244 \\
$\mathrm{C}$ & 1.482585 & -2.317624 & -0.241167 \\
$\mathrm{H}$ & -0.671058 & -2.263122 & -0.210353 \\
$\mathrm{H}$ & 0.087565 & -1.477903 & 1.199898 \\
$\mathrm{C}$ & 1.626450 & -3.672998 & 0.425897 \\
$\mathrm{H}$ & 1.562118 & -2.437678 & -1.341474 \\
$\mathrm{H}$ & 2.341874 & -1.680255 & 0.054113 \\
$\mathrm{C}$ & 2.936541 & -4.355039 & 0.078705 \\
$\mathrm{H}$ & 1.542196 & -3.548479 & 1.524107 \\
$\mathrm{H}$ & 0.773360 & -4.315157 & 0.127687 \\
$\mathrm{H}$ & 3.037228 & -5.337099 & 0.572848 \\
$\mathrm{H}$ & 3.021262 & -4.517190 & -1.011815 \\
$\mathrm{H}$ & 3.799774 & -3.735588 & 0.385595 \\
$\mathrm{C}$ & 1.273364 & 0.964564 & 0.153499 \\
$\mathrm{C}$ & 1.306944 & 2.427112 & -0.248312 \\
$\mathrm{H}$ & 2.253656 & 0.496303 & -0.068442 \\
$\mathrm{H}$ & 1.107300 & 0.864278 & 1.248109 \\
$\mathrm{C}$ & 2.400900 & 3.212333 & 0.452340 \\
$\mathrm{H}$ & 1.440575 & 2.505881 & -1.346757 \\
$\mathrm{H}$ & 0.327775 & 2.903585 & -0.030863 \\
$\mathrm{C}$ & 2.413800 & 4.674819 & 0.049122 \\
$\mathrm{H}$ & 2.269157 & 3.121339 & 1.549302 \\
$\mathrm{H}$ & 3.381505 & 2.747415 & 0.225636 \\
$\mathrm{H}$ & 3.210792 & 5.240351 & 0.562654 \\
$\mathrm{H}$ & 2.572667 & 4.783309 & -1.039780 \\
$\mathrm{H}$ & 1.449752 & 5.160533 & 0.289243 \\
$\mathrm{C}$ & -1.507815 & 0.621591 & 0.156131
\end{tabular}




$\begin{array}{lrrc}\mathrm{C} & -2.783063 & -0.098779 & -0.240728 \\ \mathrm{H} & -1.610081 & 1.702999 & -0.067508 \\ \mathrm{H} & -1.332303 & 0.532885 & 1.250036 \\ \mathrm{C} & -4.015328 & 0.441548 & 0.461324 \\ \mathrm{H} & -2.923666 & -0.026616 & -1.338875 \\ \mathrm{H} & -2.691094 & -1.183081 & -0.020557 \\ \mathrm{C} & -5.281839 & -0.287252 & 0.053548 \\ \mathrm{H} & -3.871302 & 0.368608 & 1.558013 \\ \mathrm{H} & -4.112095 & 1.523542 & 0.239861 \\ \mathrm{H} & -6.174418 & 0.113145 & 0.565082 \\ \mathrm{H} & -5.453087 & -0.203977 & -1.035744 \\ \mathrm{H} & -5.213621 & -1.364866 & 0.292526\end{array}$

$\begin{array}{cccc}\mathbf{P h}_{3} \mathbf{P} & & & \\ \mathrm{P} & 0.000369 & -0.004156 & -1.316219 \\ \mathrm{C} & 3.695977 & -1.888047 & 0.747135 \\ \mathrm{C} & 1.463966 & -0.696847 & -0.467415 \\ \mathrm{C} & 3.100937 & -2.488239 & -0.366643 \\ \mathrm{C} & 1.997889 & -1.889497 & -0.975410 \\ \mathrm{C} & 2.065130 & -0.099932 & 0.647416 \\ \mathrm{C} & 3.178321 & -0.691814 & 1.247815 \\ \mathrm{H} & 4.569616 & -2.351070 & 1.220536 \\ \mathrm{H} & 3.506922 & -3.423837 & -0.769158 \\ \mathrm{H} & 3.643735 & -0.216407 & 2.119649 \\ \mathrm{H} & 1.540671 & -2.351931 & -1.860631 \\ \mathrm{H} & 1.652442 & 0.836398 & 1.045500 \\ \mathrm{C} & -3.492926 & -2.243990 & 0.742848 \\ \mathrm{C} & -1.335606 & -0.924136 & -0.473449 \\ \mathrm{C} & -3.712317 & -1.409770 & -0.357558 \\ \mathrm{C} & -2.637771 & -0.762538 & -0.967312 \\ \mathrm{C} & -1.121989 & -1.761517 & 0.628066 \\ \mathrm{C} & -2.196020 & -2.421776 & 1.228789 \\ \mathrm{H} & -4.334833 & -2.761582 & 1.217166 \\ \mathrm{H} & -4.727359 & -1.271201 & -0.748340 \\ \mathrm{H} & -2.019054 & -3.077072 & 2.090168 \\ \mathrm{H} & -2.808437 & -0.119315 & -1.841187 \\ \mathrm{H} & -0.103006 & -1.892009 & 1.015888 \\ \mathrm{C} & -0.199462 & 4.142228 & 0.739287 \\ \mathrm{C} & -0.133677 & 1.613142 & -0.474928 \\ \mathrm{C} & 0.643246 & 3.911194 & -0.352126 \\ \mathrm{C} & 0.667176 & 2.656428 & -0.960668 \\ \mathrm{C} & -0.975260 & 1.850373 & 0.618153 \\ \mathrm{C} & -1.010521 & 3.111227 & 1.217737 \\ \mathrm{H} & -0.227754 & 5.130383 & 1.213049 \\ \mathrm{H} & 1.278552 & 4.717896 & -0.736614 \\ \mathrm{H} & -1.673611 & 3.288521 & 2.073070\end{array}$




$\begin{array}{rrrr}\mathrm{H} & 1.318643 & 2.478503 & -1.826961 \\ \mathrm{H} & -1.604619 & 1.036130 & 1.000739\end{array}$

PdL

\section{Pd(TTBP)}

$\begin{array}{llll}\mathrm{P} & -0.136381 & 0.000175 & 0.000450\end{array}$

C $\quad-0.800467-1.268949-1.246951$

C $\quad-2.265848 \quad-1.647428 \quad-1.085264$

C $\quad-0.572068-0.713966-2.647256$

C $\quad 0.071637 \quad-2.518595 \quad-1.150527$

C $\quad-0.806522 \quad-0.454132 \quad 1.719103$

C $\quad-2.277796 \quad-0.144586 \quad 1.954003$

$\begin{array}{llll}\text { C } & -0.560307 & -1.940936 & 1.937992\end{array}$

$\begin{array}{llll}\text { C } & 0.049666 & 0.266182 & 2.757248\end{array}$

$\begin{array}{llll}\text { C } & -0.822907 & 1.708969 & -0.469855\end{array}$

C $\quad-2.289574 \quad 1.742973 \quad-0.874853$

C $\quad-0.602252 \quad 2.648463 \quad 0.708849$

C $\quad 0.041796 \quad 2.258395 \quad-1.601972$

$\mathrm{H} \quad 1.143023 \quad-2.229798 \quad-1.182081$

$\mathrm{H} \quad-0.154782-3.176361 \quad-2.013094$

$\mathrm{H} \quad-0.092017 \quad-3.097385 \quad-0.228431$

H $\quad 0.478782 \quad-0.388038 \quad-2.771927$

$\mathrm{H} \quad-1.240931 \quad 0.128317 \quad-2.894266$

$\mathrm{H} \quad-0.770659 \quad-1.522395 \quad-3.377784$

$\mathrm{H} \quad-2.460313-2.178324 \quad-0.136567$

$\mathrm{H} \quad-2.546243 \quad-2.337785-1.905557$

H $\quad-2.941357 \quad-0.775903 \quad-1.139751$

$\begin{array}{llll}\mathrm{H} & 1.124124 & 0.087789 & 2.544578\end{array}$

$\mathrm{H} \quad-0.195971 \quad-0.135495 \quad 3.760451$

H $\quad-0.110699 \quad 1.355126 \quad 2.776683$

$\mathrm{H} \quad 0.492317 \quad-2.199693 \quad 1.711391$

H $\quad-1.225783 \quad-2.5841341 .336496$

$\mathrm{H} \quad-0.748955 \quad-2.172374 \quad 3.004630$

$\mathrm{H} \quad-2.486328 \quad 0.939987 \quad 1.941619$

H $\quad-2.563637 \quad-0.516606 \quad 2.957954$

$\mathrm{H} \quad-2.941144 \quad-0.632872 \quad 1.218683$

H $\quad 1.113636 \quad 2.177724 \quad-1.326034$

$\mathrm{H} \quad-0.215567 \quad 3.324843 \quad-1.759634$

$\mathrm{H} \quad-0.097092 \quad 1.728878 \quad-2.557357$

H $\quad 0.449773 \quad 2.606127 \quad 1.051358$

$\mathrm{H} \quad-1.267415 \quad 2.437855 \quad 1.563904$

$\mathrm{H} \quad-0.811392 \quad 3.682726 \quad 0.371789$

$\mathrm{H} \quad-2.482948 \quad 1.177820-1.803627$

$\mathrm{H} \quad-2.578616 \quad 2.794840 \quad-1.069859$

$\mathrm{H} \quad-2.959606 \quad 1.355407 \quad-0.087400$ 


\section{$\begin{array}{llll}\text { Pd } & 2.024856 & 0.011495 & 0.000087\end{array}$}

\section{Pd(DTBNpP)}

P 0.407935

C 1.342879

$0.195914 \quad 0.011149$

$0.494445 \quad 1.616706$

$\begin{array}{llll}\text { C } & 1.990761 & 1.863920 & 1.719825\end{array}$

$\begin{array}{llll}\text { C } & 0.327630 & 0.319322 & 2.736663\end{array}$

$\begin{array}{llll}\text { C } & 2.387714 & -0.601903 & 1.776484\end{array}$

$\begin{array}{llll}\text { C } & 1.414581 & 0.793445 & -1.464764\end{array}$

$\begin{array}{llll}\text { C } & 1.630631 & 2.296866 & -1.551506\end{array}$

$\begin{array}{llll}\text { C } & 2.755650 & 0.078073 & -1.463730\end{array}$

$\begin{array}{llll}\text { C } & 0.622759 & 0.333706 & -2.681897\end{array}$

$\begin{array}{llll}C & -2.427515 & 0.869698 & 0.054080\end{array}$

$\begin{array}{llll}\text { C } & -2.705132 & 0.139761 & -1.250461\end{array}$

$\begin{array}{llll}\text { C } & -2.684339 & -0.085665 & 1.207378\end{array}$

$\begin{array}{llll}\mathrm{C} & -3.358120 & 2.068589 & 0.173719\end{array}$

$\begin{array}{llll}\mathrm{H} & 1.909132 & -1.594889 & 1.656614\end{array}$

$\begin{array}{llll}\mathrm{H} & 2.838536 & -0.534424 & 2.786166\end{array}$

$\begin{array}{llll}\mathrm{H} & 3.198803 & -0.528881 & 1.032825\end{array}$

$\begin{array}{llll}\mathrm{H} & -0.193751 & -0.652720 & 2.640289\end{array}$

$\begin{array}{llll}\mathrm{H} & -0.433960 & 1.120044 & 2.754921\end{array}$

$\begin{array}{llll}\mathrm{H} & 0.860237 & 0.338020 & 3.707264\end{array}$

$\begin{array}{llll}\mathrm{H} & 2.848950 & 1.966729 & 1.031269\end{array}$

$\begin{array}{llll}\mathrm{H} & 2.378842 & 2.005429 & 2.747881\end{array}$

$\begin{array}{llll}\mathrm{H} & 1.280023 & 2.688241 & 1.519526\end{array}$

$\begin{array}{llll}\mathrm{H} & 0.464297 & -0.763048 & -2.645012\end{array}$

$\begin{array}{llll}\mathrm{H} & 1.180641 & 0.596531 & -3.602428\end{array}$

$\begin{array}{llll}\mathrm{H} & -0.374587 & 0.810098 & -2.738592\end{array}$

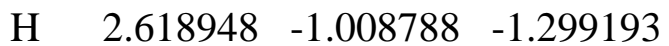

$\begin{array}{llll}\mathrm{H} & 3.435375 & 0.470953 & -0.685490\end{array}$

$\begin{array}{llll}\mathrm{H} & 3.250515 & 0.224278 & -2.443787\end{array}$

$\begin{array}{llll}\mathrm{H} & 0.682021 & 2.861317 & -1.606159\end{array}$

$\begin{array}{llll}\mathrm{H} & 2.188810 & 2.520409 & -2.481791\end{array}$

$\begin{array}{llll}\mathrm{H} & 2.217126 & 2.695755 & -0.707605\end{array}$

$\begin{array}{llll}\mathrm{H} & -3.192681 & 2.609206 & 1.125085\end{array}$

$\begin{array}{llll}\mathrm{H} & -4.415200 & 1.744863 & 0.148097\end{array}$

$\begin{array}{llll}\mathrm{H} & -3.202368 & 2.783462 & -0.656841\end{array}$

$\begin{array}{llll}\mathrm{H} & -2.506749 & 0.392441 & 2.188783\end{array}$

$\begin{array}{llll}\mathrm{H} & -2.004135 & -0.969579 & 1.114428\end{array}$

$\begin{array}{llll}\mathrm{H} & -3.729790 & -0.444894 & 1.179752\end{array}$

$\begin{array}{llll}\mathrm{H} & -2.574347 & 0.805410 & -2.125852\end{array}$

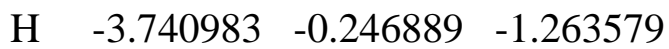

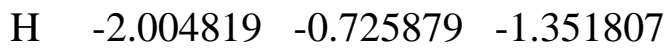

$\begin{array}{llll}\text { C } & -0.988425 & 1.411155 & 0.063049\end{array}$

$\begin{array}{llll}\mathrm{H} & -0.842898 & 2.058320 & 0.952535\end{array}$

H $\quad-0.874272 \quad 2.071386 \quad-0.820053$ 


\begin{tabular}{|c|c|c|c|}
\hline & -0.200765 & -1.872176 & -0.172560 \\
\hline \multicolumn{4}{|c|}{ Pd(TBDNpP) } \\
\hline & 0.027947 & 0.472701 & 0.264608 \\
\hline & 2.553648 & -0.875577 & 0.720084 \\
\hline 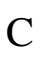 & 1.819455 & -2.205947 & 0.687436 \\
\hline 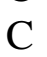 & 3.099590 & -0.569522 & -0.665297 \\
\hline $\mathrm{C}$ & 3.704711 & -0.952391 & 1.712614 \\
\hline C & -0.263983 & 2.307369 & 0.503410 \\
\hline $\mathrm{C}$ & -0.091057 & 2.731760 & 1.953112 \\
\hline $\mathrm{C}$ & 0.733570 & 3.030870 & -0.387146 \\
\hline $\mathrm{C}$ & -1.668008 & 2.634525 & 0.028196 \\
\hline $\mathrm{C}$ & -2.307404 & -1.218025 & 0.737618 \\
\hline $\mathrm{C}$ & -3.149775 & -0.421148 & 4535 \\
\hline $\mathrm{C}$ & -1.711396 & -2.408844 & 0.003797 \\
\hline $\mathrm{C}$ & -3.189779 & -1.720139 & 1.872681 \\
\hline $\mathrm{H}$ & 4.248017 & 0.009689 & 1.770941 \\
\hline $\mathrm{H}$ & 4.428078 & -1.731754 & 1.408872 \\
\hline $\mathrm{H}$ & 3.341575 & -1.202140 & 2.727900 \\
\hline $\mathrm{H}$ & 3.692758 & 0.365127 & 6204 \\
\hline $\mathrm{H}$ & 2.247556 & -0.438054 & -1.383597 \\
\hline $\mathrm{H}$ & 3.742989 & -1.393292 & -1.025355 \\
\hline $\mathrm{H}$ & 1.365142 & -2.445427 & 1.669161 \\
\hline $\mathrm{H}$ & 2.514761 & -3.025074 & 0.425470 \\
\hline $\mathrm{H}$ & 1.015364 & -2.177450 & -0.076424 \\
\hline $\mathrm{H}$ & -1.819739 & 2.285269 & -1.011228 \\
\hline $\mathrm{H}$ & -1.822728 & 3.730507 & 0.060819 \\
\hline $\mathrm{H}$ & -2.443197 & 2.165777 & 0.662396 \\
\hline $\mathrm{H}$ & 0.619176 & 2.694986 & -1.436355 \\
\hline $\mathrm{H}$ & 1.782146 & 2.843817 & -0.088783 \\
\hline $\mathrm{H}$ & 0.558534 & 4.123044 & -0.334356 \\
\hline $\mathrm{H}$ & -0.788613 & 2.204466 & 2.630910 \\
\hline $\mathrm{H}$ & -0.302475 & 3.814762 & 2.047473 \\
\hline $\mathrm{H}$ & 0.937182 & 2.561849 & 2.318749 \\
\hline $\mathrm{H}$ & -2.606363 & -2.315656 & 2.600253 \\
\hline $\mathrm{H}$ & -3.999049 & -2.363056 & 1.479327 \\
\hline $\mathrm{H}$ & -3.659086 & -0.878596 & 2.417348 \\
\hline $\mathrm{H}$ & -1.075844 & -3.023849 & 0.669043 \\
\hline $\mathrm{H}$ & -1.079521 & -2.046610 & -0.848372 \\
\hline $\mathrm{H}$ & -2.515008 & -3.048729 & -0.405338 \\
\hline $\mathrm{H}$ & -3.647521 & 0.439895 & 0.240164 \\
\hline $\mathrm{H}$ & -3.933274 & -1.066576 & -0.683141 \\
\hline $\mathrm{H}$ & -2.504794 & -0.043411 & -1.070415 \\
\hline $\mathrm{C}$ & -1.205686 & -0.355004 & 1.370666 \\
\hline $\mathrm{H}$ & -0.621991 & -0.991786 & 2.065972 \\
\hline 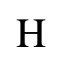 & -1.679079 & 0.435127 & 1.991098 \\
\hline
\end{tabular}




$\begin{array}{lrrr}\mathrm{C} & 1.619662 & 0.249053 & 1.179413 \\ \mathrm{H} & 1.377424 & 0.115971 & 2.254672 \\ \mathrm{H} & 2.167881 & 1.209439 & 1.091674 \\ \mathrm{Pd} & 0.008244 & -0.201319 & -1.789219\end{array}$

\section{Pd(TNpP)}

$\begin{array}{llll}\mathrm{P} & 0.000000 & 0.000000 & -0.488098\end{array}$

C $\quad 1.321729 \quad 2.561929-0.565284$

$\begin{array}{llll}\text { C } & 0.000000 & 3.206692 & -0.174107\end{array}$

C $\quad 2.145760 \quad 2.313382 \quad 0.688663$

$\begin{array}{llll}\text { C } & 2.087301 & 3.490738 & -1.496101\end{array}$

C $\quad 1.557831-2.425615 \quad-0.565284$

C $\quad 1.979418-3.553025 \quad-1.496101$

C $\quad 2.777076-1.603346 \quad-0.174107$

C $\quad 0.930568$-3.014973 0.688663

$\begin{array}{llll}\text { C } & -2.879560 & -0.136313 & -0.565284\end{array}$

$\begin{array}{llll}\text { C } & -2.777076 & -1.603346 & -0.174107\end{array}$

$\begin{array}{llll}\text { C } & -3.076327 & 0.701591 & 0.688663\end{array}$

C $\quad-4.066718 \quad 0.062286 \quad-1.496101$

$\mathrm{H} \quad 3.048531 \quad 3.039791 \quad-1.806951$

$\mathrm{H} \quad 2.310646 \quad 4.450472 \quad-0.994008$

$\mathrm{H} \quad 1.502735 \quad 3.711805 \quad-2.409480$

H $3.136962 \quad 1.888673 \quad 0.439086$

$\mathrm{H} \quad 1.611700 \quad 1.581420 \quad 1.351765$

H $\quad 2.298166 \quad 3.252774 \quad 1.251429$

H $\quad-0.638898 \quad 3.396497 \quad-1.058865$

$\mathrm{H} \quad 0.179286 \quad 4.176170 \quad 0.326747$

$\mathrm{H} \quad-0.551983 \quad 2.553254 \quad 0.534901$

$\mathrm{H} \quad 0.563700 \quad-2.186483 \quad 1.351765$

$\mathrm{H} \quad 1.667902 \quad-3.616657 \quad 1.251429$

H $\quad 0.067158 \quad-3.661025 \quad 0.439086$

$\mathrm{H} \quad 2.487174 \quad-0.798596 \quad 0.534901$

H $\quad 3.260902 \quad-1.144947 \quad-1.058865$

H $\quad 3.527026 \quad-2.243351 \quad 0.326747$

$\mathrm{H} \quad 1.108271 \quad-4.160001 \quad-1.806951$

H $\quad 2.698899 \quad-4.226314-0.994008$

H $\quad 2.463150 \quad-3.157310-2.409480$

$\mathrm{H} \quad-4.156802 \quad 1.120210 \quad-1.806951$

H $\quad-5.009544 \quad-0.224158 \quad-0.994008$

$\mathrm{H} \quad-3.965885-0.554496-2.409480$

$\mathrm{H} \quad-3.204119 \quad 1.7723520 .439086$

$\mathrm{H} \quad-2.175400 \quad 0.605063 \quad 1.351765$

$\mathrm{H} \quad-3.966068 \quad 0.363883 \quad 1.251429$

$\mathrm{H} \quad-2.622004 \quad-2.251551 \quad-1.058865$

$\mathrm{H} \quad-3.706312 \quad-1.932819 \quad 0.326747$

$\mathrm{H} \quad-1.935191 \quad-1.754658 \quad 0.534901$ 


$\begin{array}{lrrr}\mathrm{C} & -1.620346 & 0.320288 & -1.310606 \\ \mathrm{H} & -1.675682 & 1.414807 & -1.474797 \\ \mathrm{H} & -1.580781 & -0.156537 & -2.313508 \\ \mathrm{C} & 1.087550 & 1.243117 & -1.310606 \\ \mathrm{H} & 0.654825 & 1.447265 & -2.313508 \\ \mathrm{H} & 2.063099 & 0.743779 & -1.474797 \\ \mathrm{C} & 0.532796 & -1.563405 & -1.310606 \\ \mathrm{H} & -0.387418 & -2.158586 & -1.474797 \\ \mathrm{H} & 0.925956 & -1.290728 & -2.313508 \\ \mathrm{Pd} & 0.000000 & 0.000000 & 1.678616\end{array}$

\section{$\mathbf{P d}\left[(\mathbf{i}-\mathbf{P r})_{3} \mathbf{P}\right]$}

P $0.32200800 \quad 0.06425100 \quad 0.04136600$

C $0.78051700 \quad 1.85399700 \quad 0.22824600$

C $0.96321400 \quad 2.47535300 \quad-1.14724200$

C - $0.32027500 \quad 2.60084000 \quad 0.96493600$

C $0.83953600 \quad-0.77446700 \quad 1.61916900$

C $0.53171600 \quad 0.05523800 \quad 2.85208300$

C $0.14384100-2.12248700 \quad 1.71906600$

C $1.56524900-0.54509400 \quad-1.19959600$

C $3.00887800-0.25791400-0.82690900$

C $1.34650800-2.01323100-1.51616700$

$\mathrm{H}-1.27816900 \quad 2.44920300 \quad 0.42703000$

$\mathrm{H}-0.09284000 \quad 3.68332400 \quad 0.99485300$

$\mathrm{H}-0.46984000 \quad 2.24776900 \quad 1.99820100$

H $0.05754900 \quad 2.29586600 \quad-1.76110600$

H $1.83593500 \quad 2.07866200 \quad-1.69508800$

H $1.09114400 \quad 3.56945900-1.05214800$

H $-0.95309800 \quad-1.96794300 \quad 1.66811000$

H $0.39652800-2.61812700 \quad 2.67590300$

H $0.40761500 \quad-2.80458900 \quad 0.89326200$

$\mathrm{H}-0.55598300 \quad 0.24693000 \quad 2.92719100$

H $1.05860500 \quad 1.02579800 \quad 2.86103200$

H $0.83329600 \quad-0.50172700 \quad 3.75900500$

H $0.27204700 \quad-2.21764200-1.68899600$

H $1.69214000-2.65924200-0.68751000$

H $1.91626300-2.30572200-2.41749300$

H $3.19086800 \quad 0.81036900-0.60807100$

H $3.68236100-0.54784600-1.65527900$

H $3.32199000 \quad-0.84341300 \quad 0.05781000$

H $1.93832600-0.91942700 \quad 1.54943400$

H $1.73145400 \quad 1.90505000 \quad 0.80106300$

H $\quad \begin{array}{llll}1.29933600 & 0.03771300 & -2.10361100\end{array}$

Pd $\quad-1.72523400 \quad-0.25134000 \quad-0.51577400$

\section{$\mathbf{P d}\left[(\mathbf{n}-\mathbf{B u})_{3} \mathbf{P}\right]$}




$\begin{array}{lrrr}\mathrm{P} & -0.001464 & 0.000741 & -0.265551 \\ \mathrm{C} & 1.525451 & -0.615780 & -1.075271 \\ \mathrm{C} & 1.953688 & -1.973735 & -0.553390 \\ \mathrm{H} & 2.317229 & 0.132074 & -0.872068 \\ \mathrm{H} & 1.363196 & -0.637109 & -2.173667 \\ \mathrm{C} & 3.290545 & -2.431634 & -1.105112 \\ \mathrm{H} & 1.991181 & -1.924822 & 0.555962 \\ \mathrm{H} & 1.182712 & -2.734914 & -0.793839 \\ \mathrm{C} & 3.695639 & -3.796386 & -0.580001 \\ \mathrm{H} & 3.245668 & -2.450275 & -2.212609 \\ \mathrm{H} & 4.063653 & -1.681309 & -0.844728 \\ \mathrm{H} & 4.671578 & -4.121582 & -0.979884 \\ \mathrm{H} & 3.769319 & -3.789003 & 0.522970 \\ \mathrm{H} & 2.948547 & -4.564457 & -0.852901 \\ \mathrm{C} & -1.302498 & -1.007198 & -1.077773 \\ \mathrm{C} & -2.691578 & -0.691355 & -0.557177 \\ \mathrm{H} & -1.056713 & -2.068053 & -0.873163 \\ \mathrm{H} & -1.237198 & -0.857760 & -2.176278 \\ \mathrm{C} & -3.759884 & -1.619654 & -1.103607 \\ \mathrm{H} & -2.668689 & -0.744333 & 0.552334 \\ \mathrm{H} & -2.961915 & 0.356920 & -0.802070 \\ \mathrm{C} & -5.142707 & -1.281465 & -0.578308 \\ \mathrm{H} & -3.754427 & -1.576779 & -2.211346 \\ \mathrm{H} & -3.498492 & -2.663557 & -0.838389 \\ \mathrm{H} & -5.915219 & -1.962725 & -0.974785 \\ \mathrm{H} & -5.172142 & -1.344488 & 0.524931 \\ \mathrm{H} & -5.430627 & -0.250320 & -0.854830 \\ \mathrm{C} & -0.227818 & 1.637996 & -1.063531 \\ \mathrm{C} & 0.743921 & 2.679882 & -0.544157 \\ \mathrm{H} & -1.267652 & 1.954683 & -0.849263 \\ \mathrm{H} & -0.138997 & 1.515320 & -2.163553 \\ \mathrm{C} & 0.469603 & 4.070936 & -1.084507 \\ \mathrm{H} & 0.694307 & 2.680746 & 0.565774 \\ \mathrm{H} & 1.785412 & 2.391870 & -0.797855 \\ \mathrm{C} & 1.460250 & 5.097418 & -0.567685 \\ \mathrm{H} & 0.492767 & 4.047335 & -2.192616 \\ \mathrm{H} & -0.562214 & 4.366998 & -0.808428 \\ \mathrm{H} & 1.252866 & 6.108126 & -0.959581 \\ \mathrm{H} & 1.432681 & 5.151638 & 0.536039 \\ \mathrm{H} & 2.493675 & 4.830841 & -0.856656 \\ \mathrm{Pd} & -0.002309 & -0.012833 & 1.872976 \\ & & \\ \end{array}$

\section{$\mathbf{P d}\left(\mathbf{P h}_{3} \mathbf{P}\right)$}

$\begin{array}{llll}\text { Pd } & 0.010005 & -0.000048 & 2.548637\end{array}$

$\begin{array}{llll}\text { P } & 0.005321 & -0.000543 & 0.417892\end{array}$

$\begin{array}{llll}\text { C } & -1.748716 & 3.776936 & -1.576932\end{array}$ 


\begin{tabular}{|c|c|c|c|}
\hline & 1 & & \\
\hline & 275585 & & \\
\hline & & & \\
\hline & & & \\
\hline & -0.654744 & & $c 00$ \\
\hline & & & \\
\hline & 744 & & \\
\hline & 1003 & & \\
\hline & 3920 & & \\
\hline & & & \\
\hline & & & \\
\hline & & & \\
\hline & & & \\
\hline & & & \\
\hline & & & \\
\hline & -2 & & \\
\hline & & & \\
\hline & & & \\
\hline & & & \\
\hline & -0 & & \\
\hline & -1 & & \\
\hline & & -0.3 & -1 \\
\hline & & & \\
\hline & & & \\
\hline & & & \\
\hline & & & \\
\hline & & & -2 \\
\hline & & & \\
\hline & & & \\
\hline & & & \\
\hline & 2.588314 & 0.92 & 1.147 \\
\hline & .924180 & -1.452374 & 50 \\
\hline
\end{tabular}




\section{${ }^{1} \mathrm{H}$ and ${ }^{13} \mathrm{C}$ NMR Spectra of Amine Products}

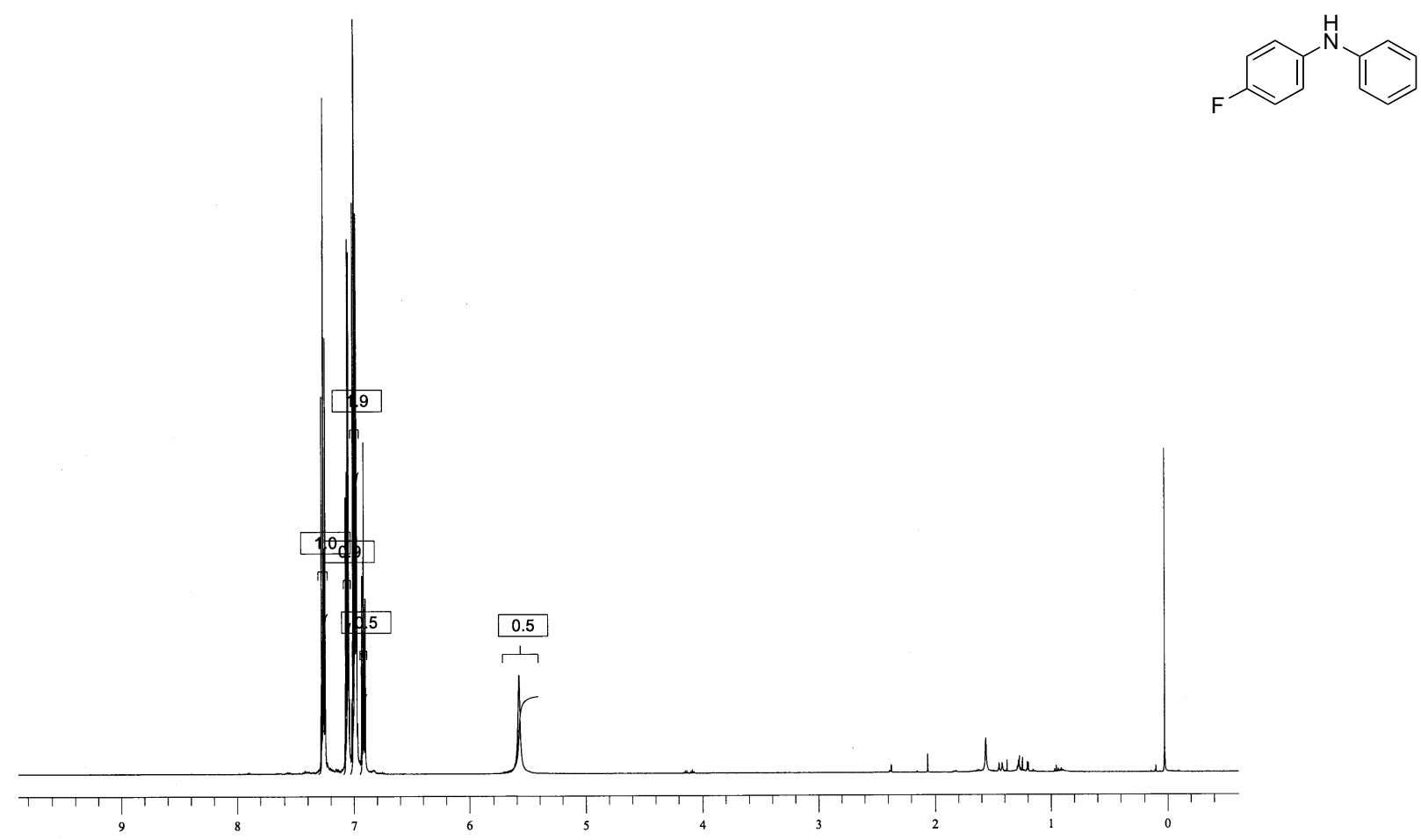

Figure S1. ${ }^{1} \mathrm{H}$ NMR spectrum $\left(\mathrm{CDCl}_{3}, 500 \mathrm{MHz}\right.$ ) of $\mathrm{N}$-(4-fluorophenyl)aniline (Table 3, entry 1).

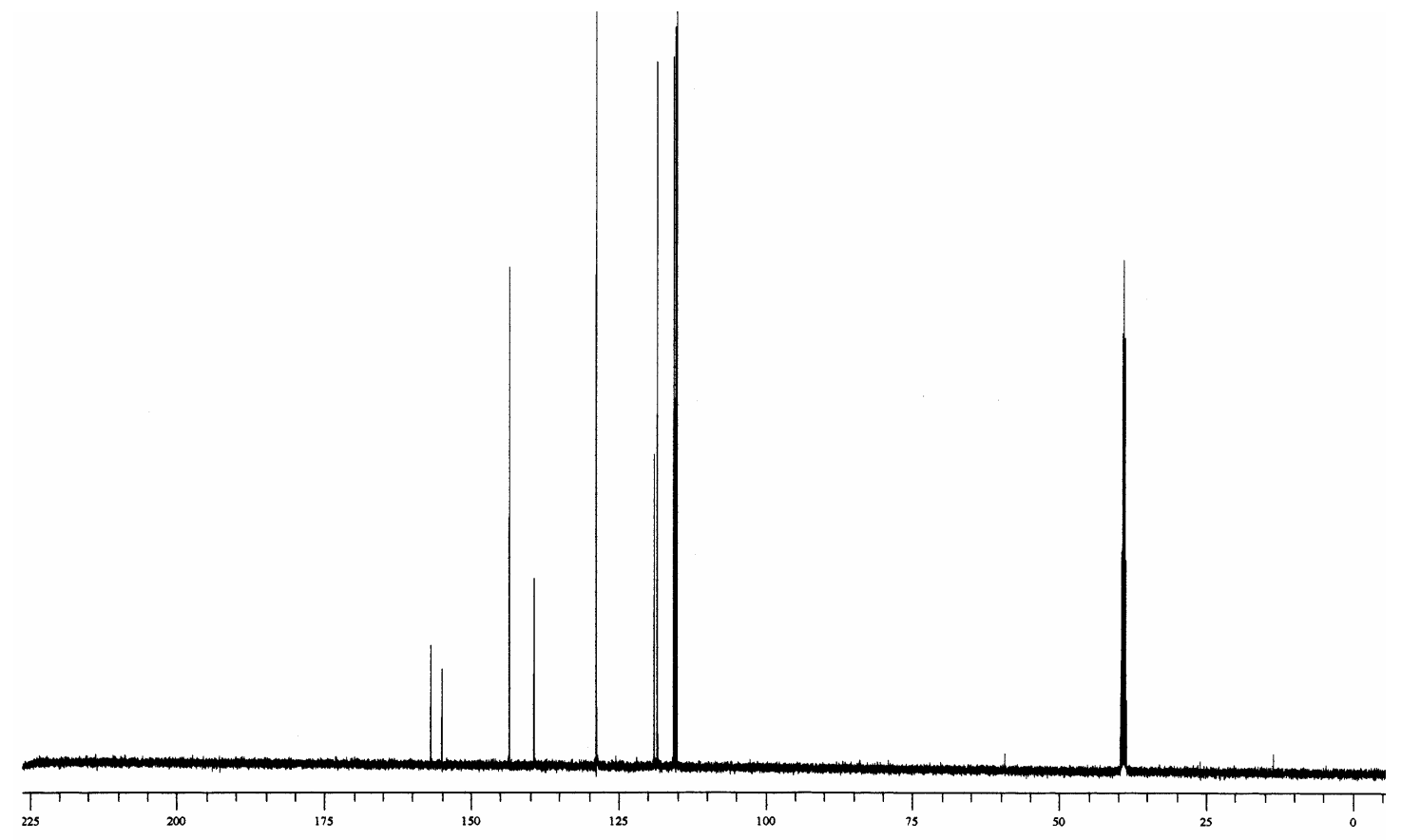

Figure S2. ${ }^{13} \mathrm{C}$ NMR spectrum (DMSO- $d_{6}, 90.6 \mathrm{MHz}$ ) of $N$-(4-fluorophenyl)aniline (Table 3, entry 1). 


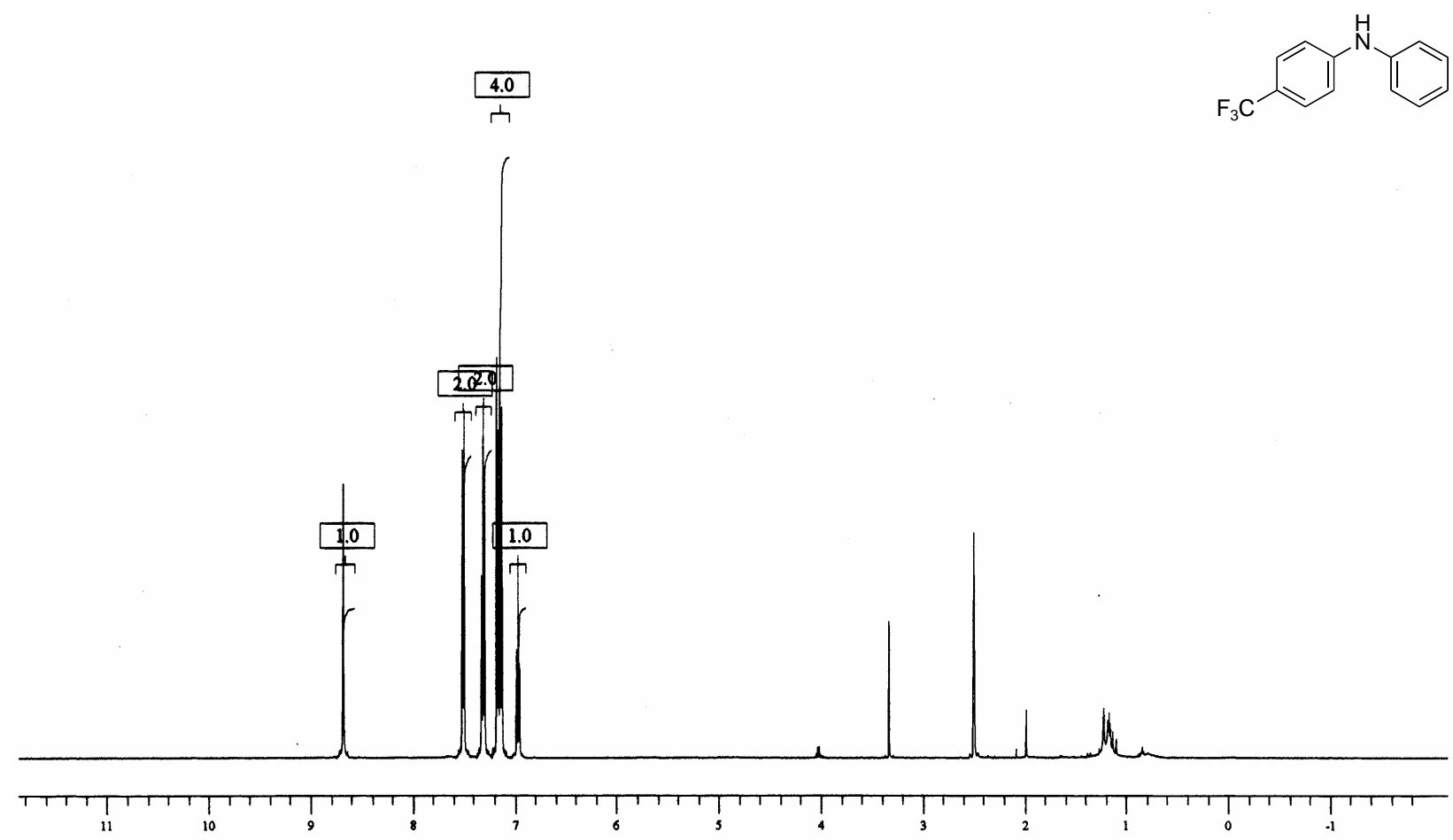

Figure S3. ${ }^{1} \mathrm{H}$ NMR spectrum (DMSO- $d_{6}, 500 \mathrm{MHz}$ ) of $N$-(4-trifluoromethylphenyl)aniline (Table 3, entry 2).

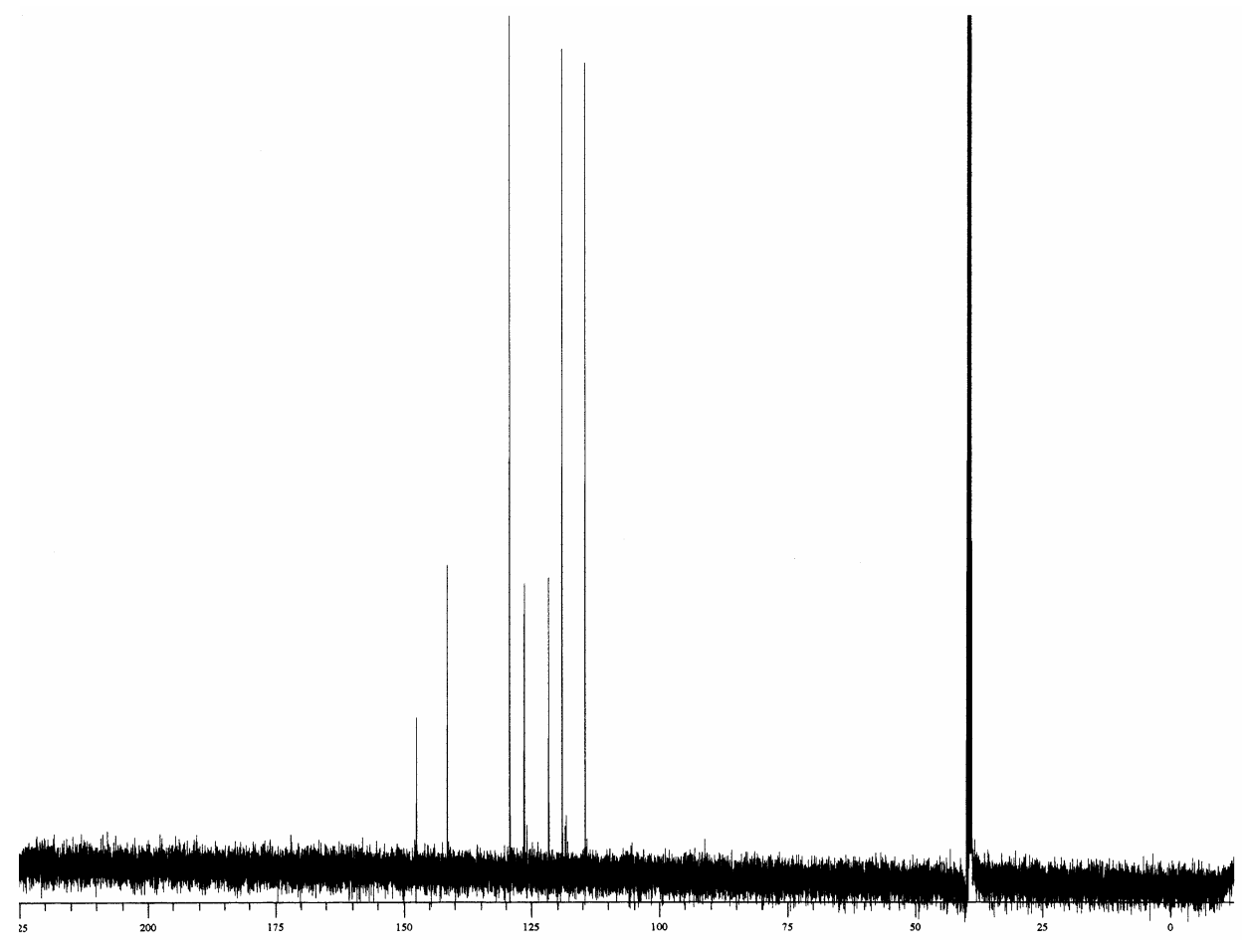

Figure S4. ${ }^{13} \mathrm{C}$ NMR spectrum (DMSO- $d_{6}, 90.6 \mathrm{MHz}$ ) of $N$-(4-trifluoromethylphenyl)aniline (Table 3, entry 2). 

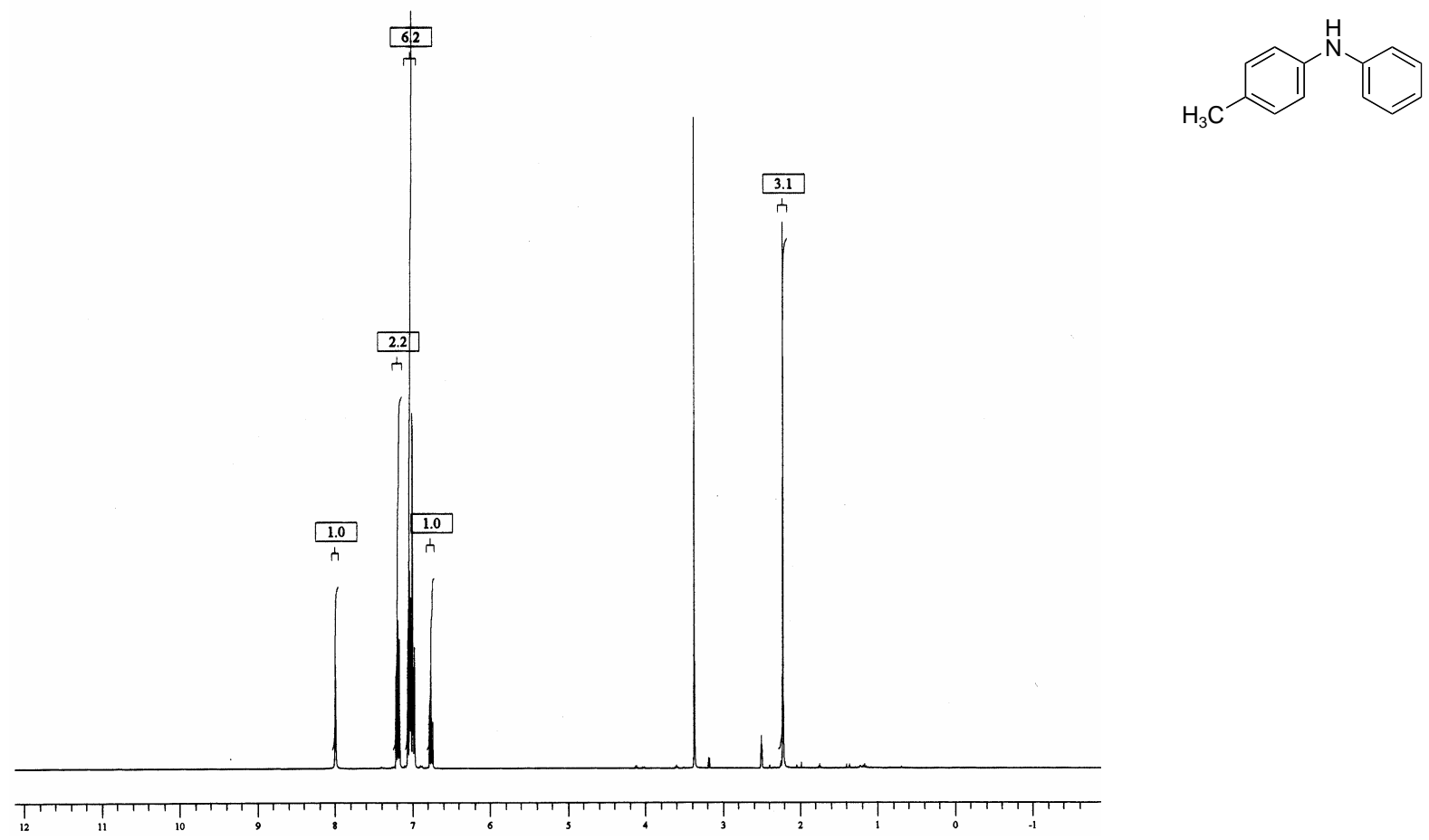

Figure S5. ${ }^{1} \mathrm{H}$ NMR spectrum (DMSO- $d_{6}, 500 \mathrm{MHz}$ ) of $N$-(4-methylphenyl)aniline (Table 3, entry 3).

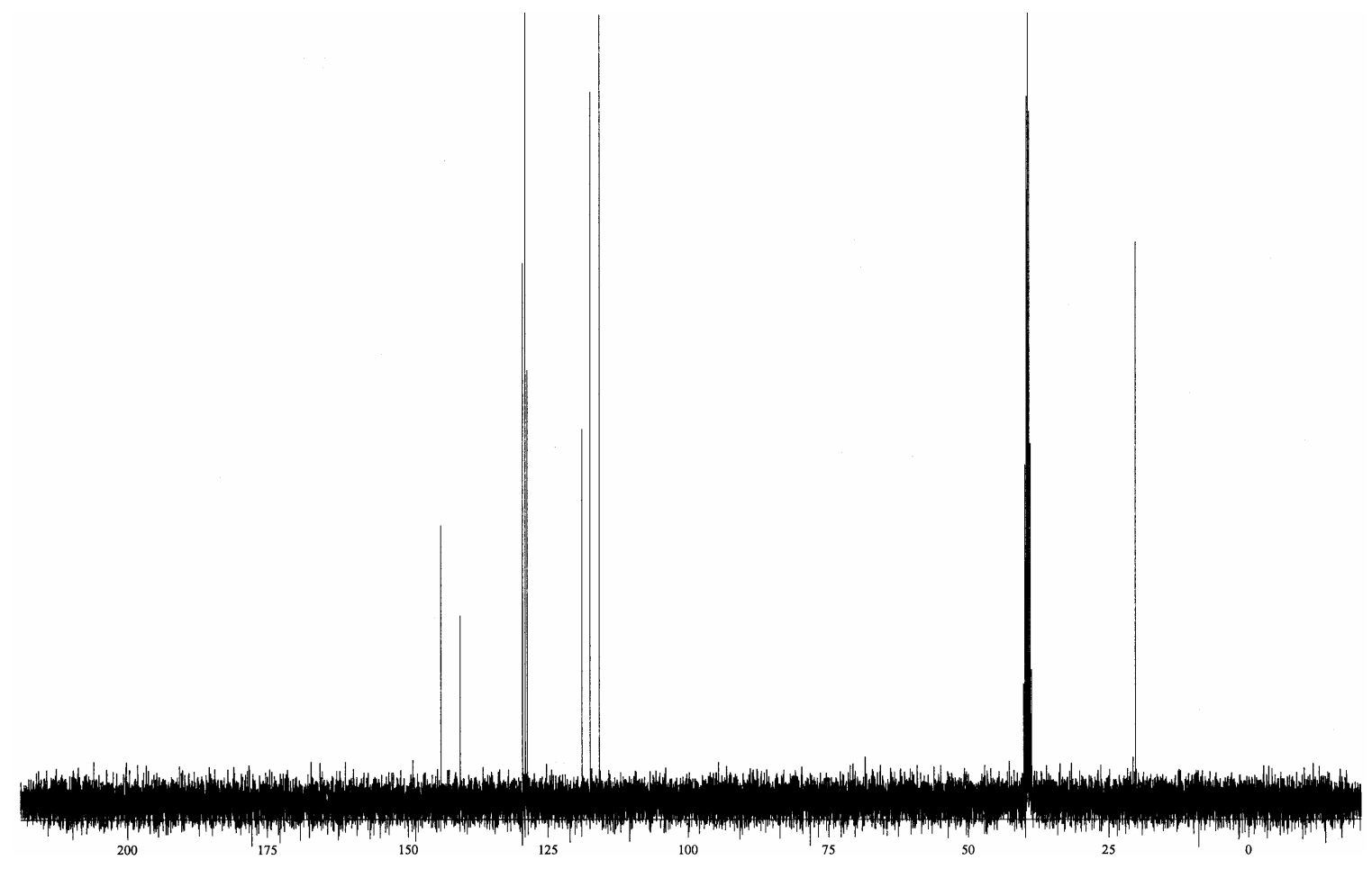

Figure S6. ${ }^{13} \mathrm{C}$ NMR spectrum (DMSO- $d_{6}, 90.6 \mathrm{MHz}$ ) of $N$-(4-methylphenyl)aniline (Table 3, entry 3). 


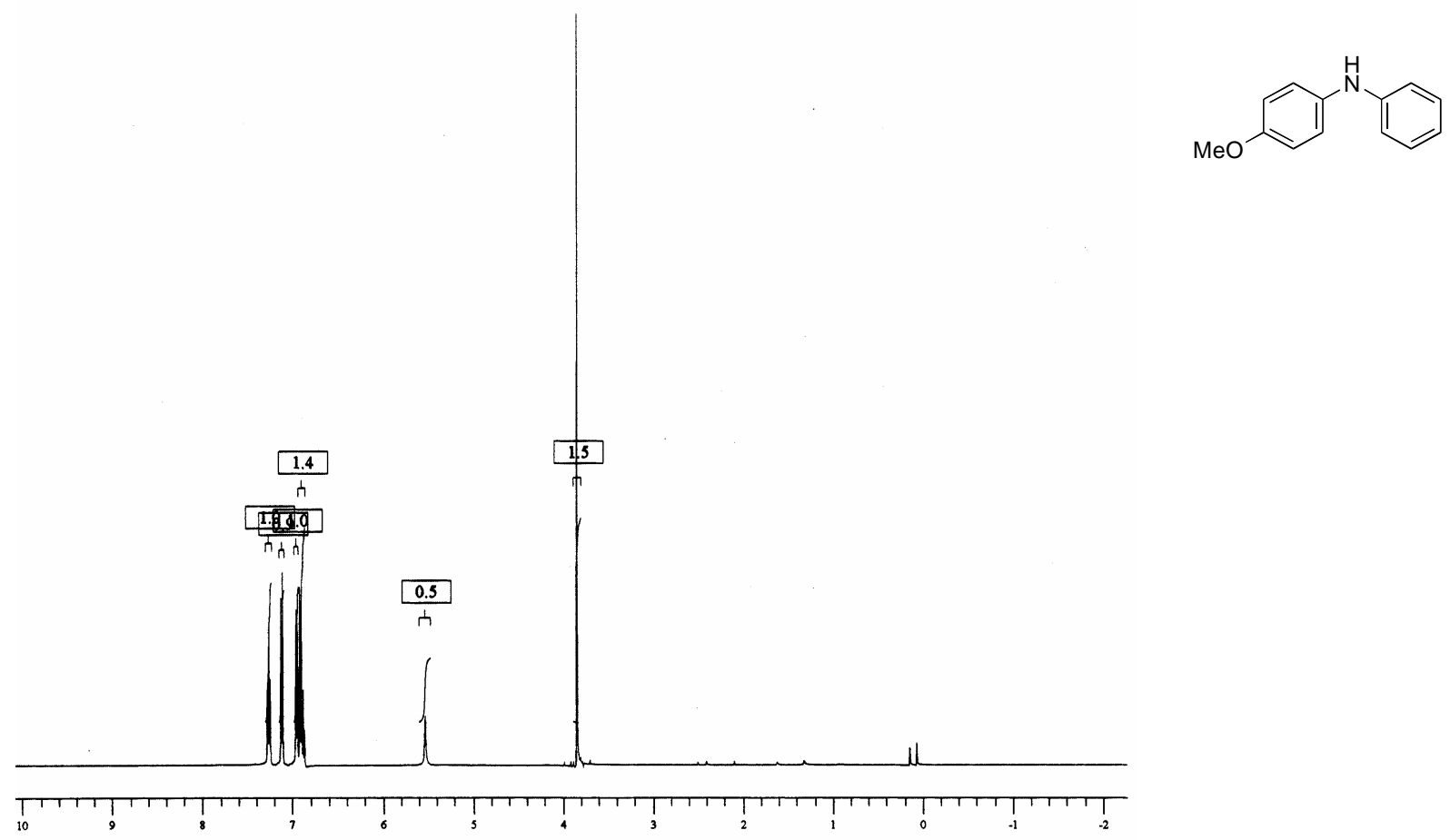

Figure S7. ${ }^{1} \mathrm{H}$ NMR spectrum $\left(\mathrm{CDCl}_{3}, 500 \mathrm{MHz}\right)$ of $N$-(4-methoxyphenyl)aniline (Table 3, entry 4).

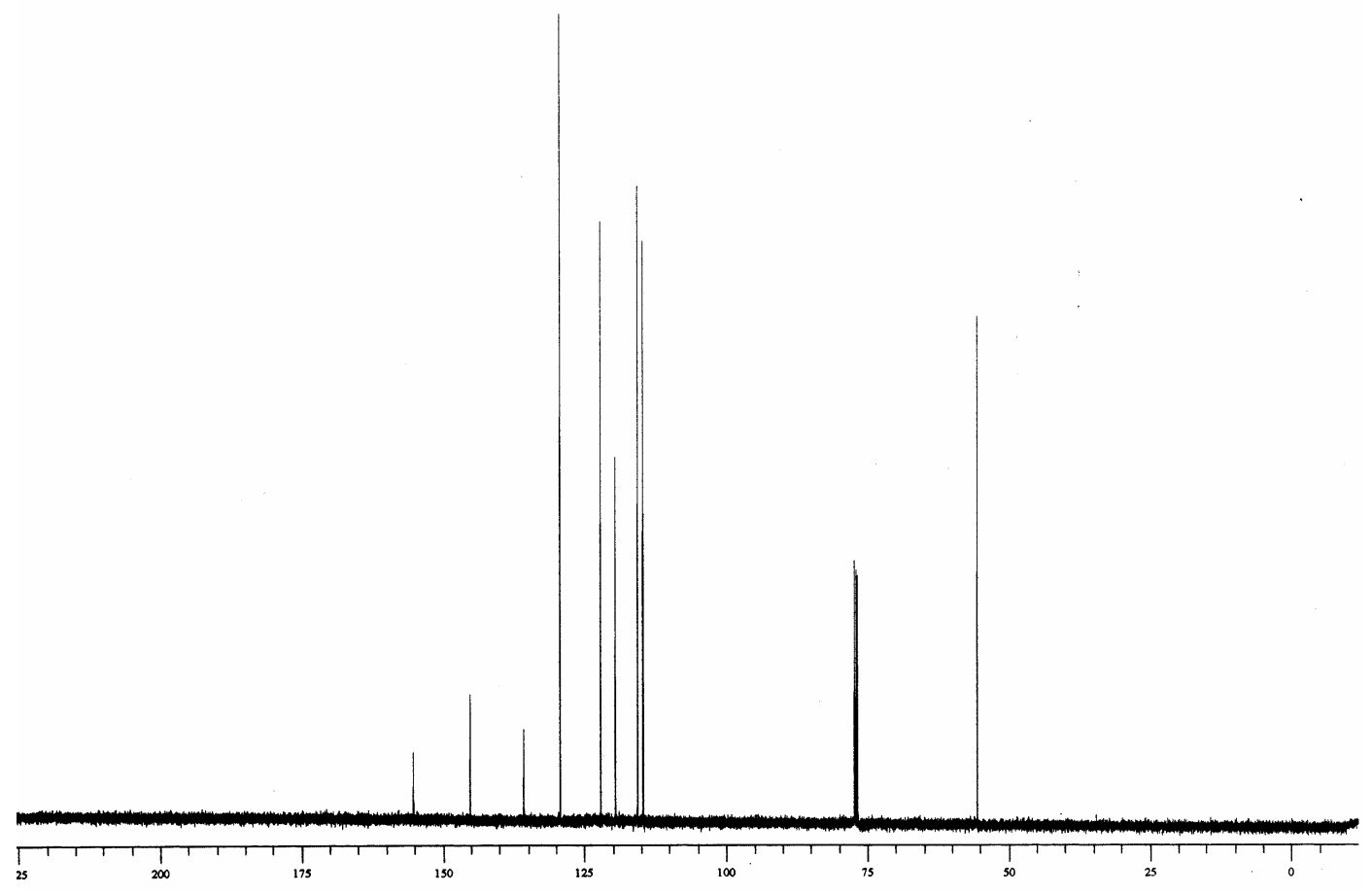

Figure S8. ${ }^{13} \mathrm{C}$ NMR spectrum $\left(\mathrm{CDCl}_{3}, 90.6 \mathrm{MHz}\right)$ of $\mathrm{N}$-(4-methoxyphenyl)aniline (Table 3, entry 4). 

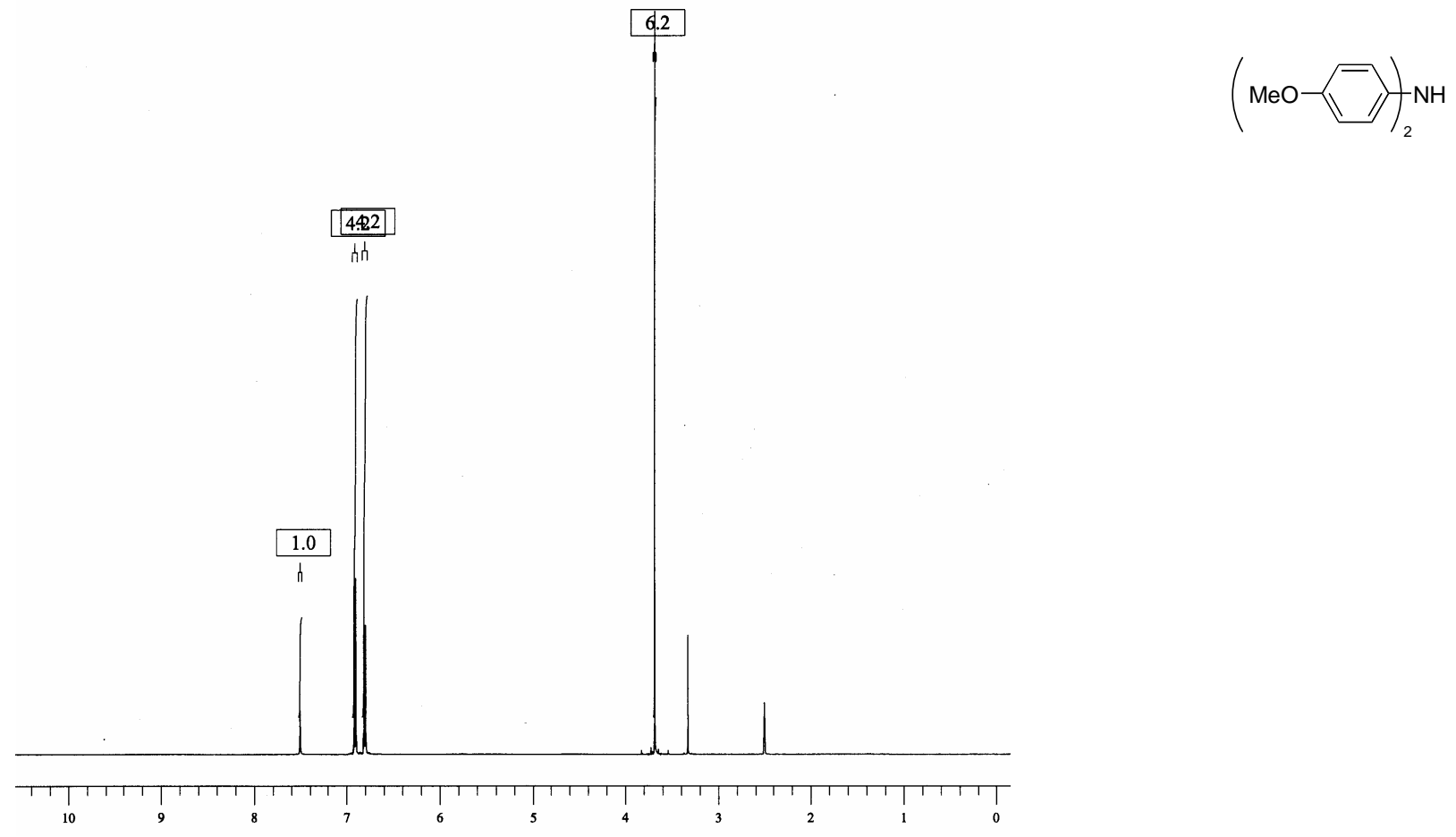

Figure S9. ${ }^{1} \mathrm{H}$ NMR spectrum (DMSO-d, $500 \mathrm{MHz}$ ) of bis(4-methoxyphenyl)amine (Table 3, entry 5).

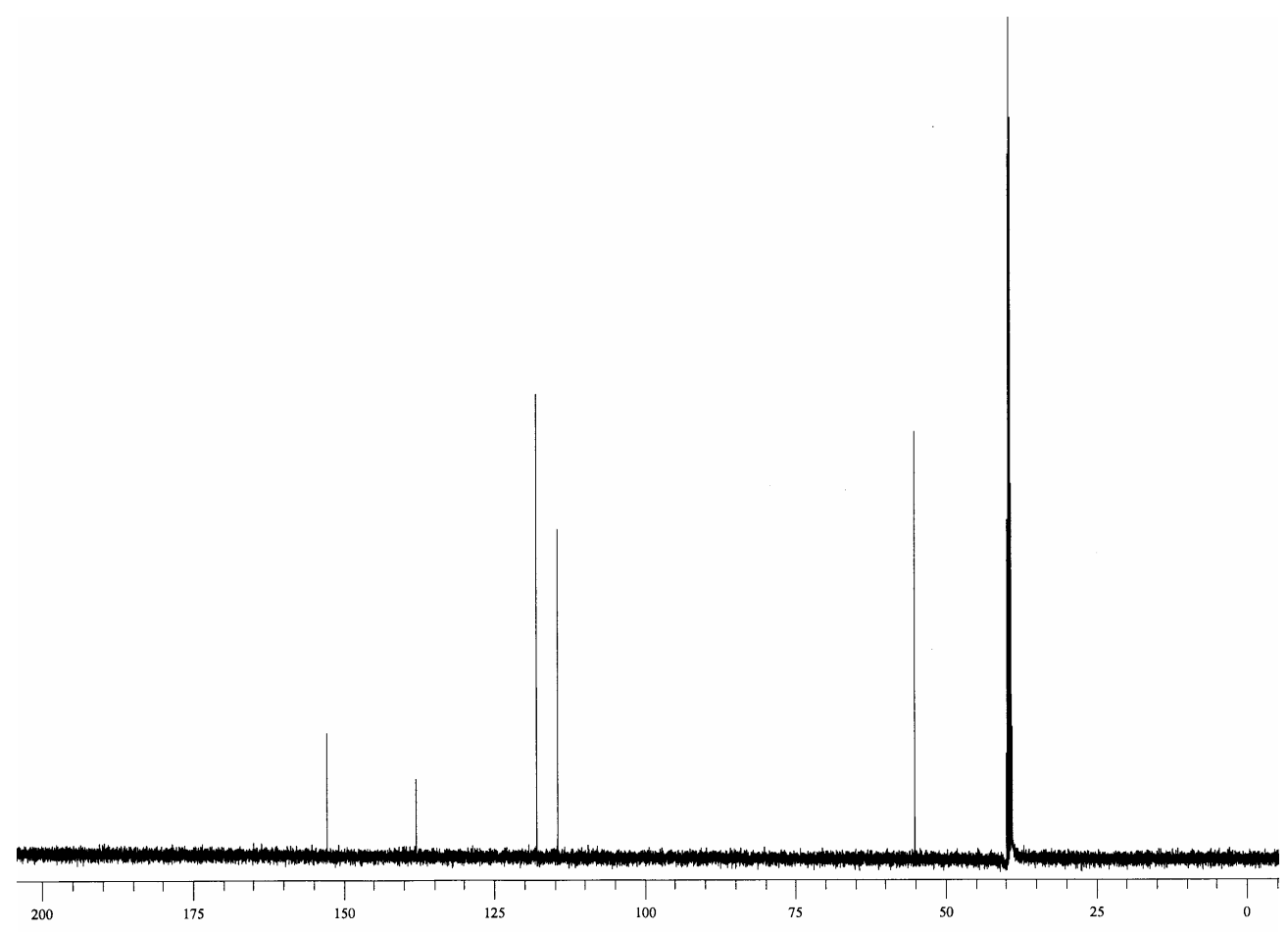

Figure S10. ${ }^{13} \mathrm{C}$ NMR spectrum (DMSO- $d_{6}, 90.6 \mathrm{MHz}$ ) of bis(4-methoxyphenyl)amine (Table 3, entry 5). 


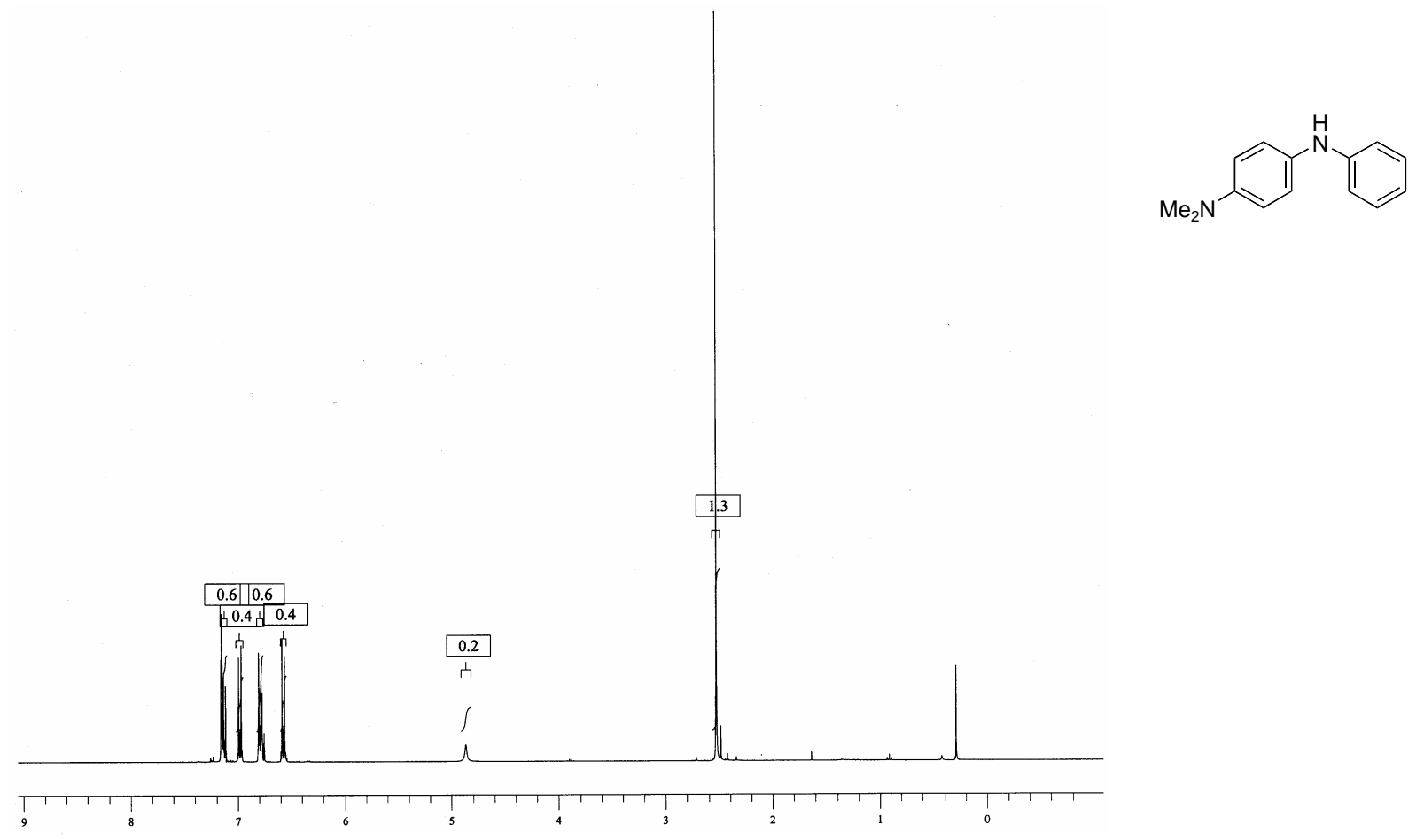

Figure S11. ${ }^{1} \mathrm{H}$ NMR spectrum $\left(\mathrm{C}_{6} \mathrm{D}_{6}, 500 \mathrm{MHz}\right)$ of $N, N$-dimethyl- $N$ '-phenyl-1,4benzenediamine (Table 3 , entry 6$)$.

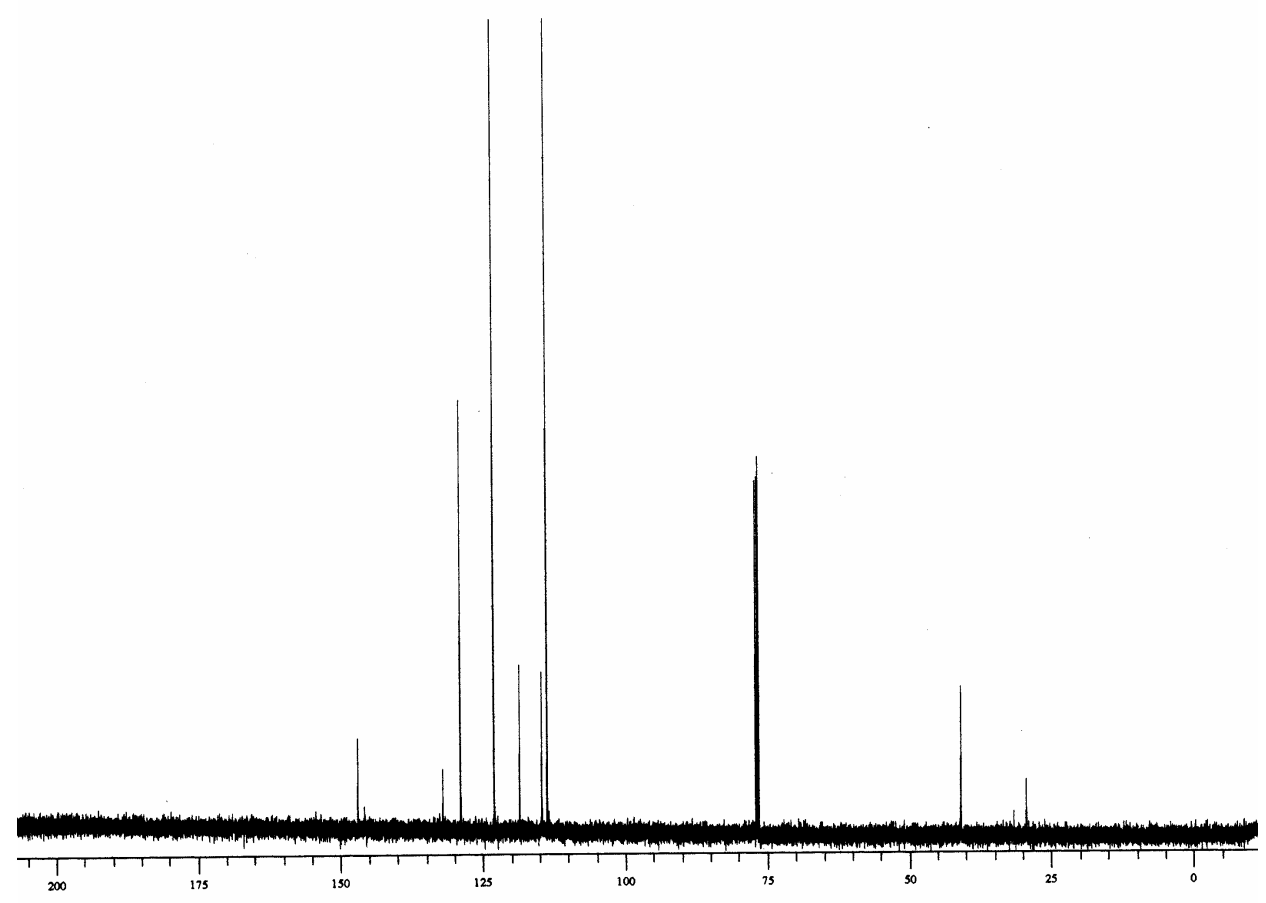

Figure S12. ${ }^{13} \mathrm{C}$ NMR spectrum $\left(\mathrm{CDCl}_{3}, 90.6 \mathrm{MHz}\right)$ of $N, N$-dimethyl- $N$-phenyl-1,4benzenediamine (Table 3 , entry 6 ). 

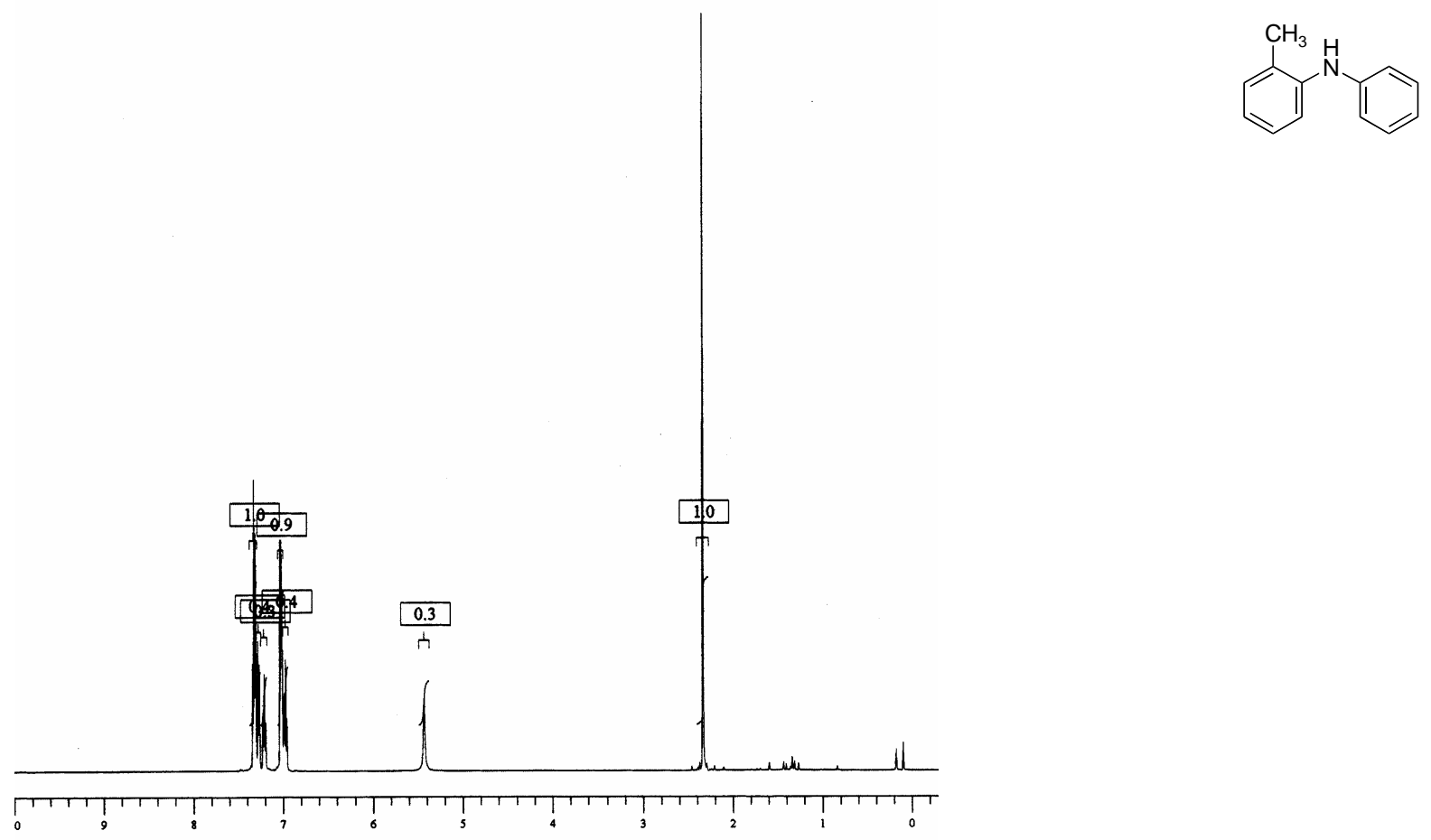

Figure S13. ${ }^{1} \mathrm{H}$ NMR spectrum $\left(\mathrm{CDCl}_{3}, 500 \mathrm{MHz}\right)$ of $N$-(2-methylphenyl)aniline (Table 3, entry 7).

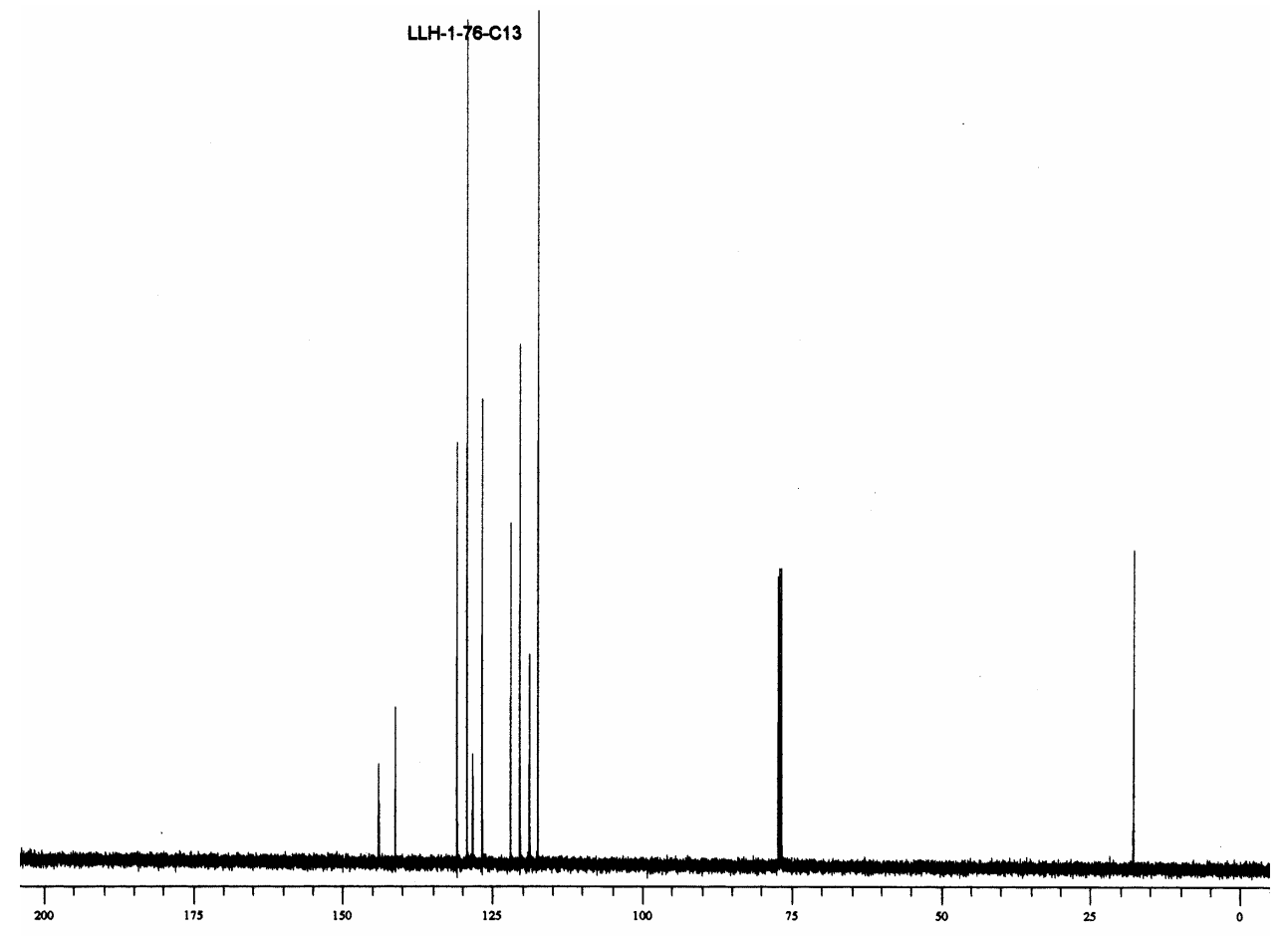

Figure S14. ${ }^{13} \mathrm{C}$ NMR spectrum $\left(\mathrm{CDCl}_{3}, 90.6 \mathrm{MHz}\right)$ of $N$-(2-methylphenyl)aniline (Table 3, entry 7). 

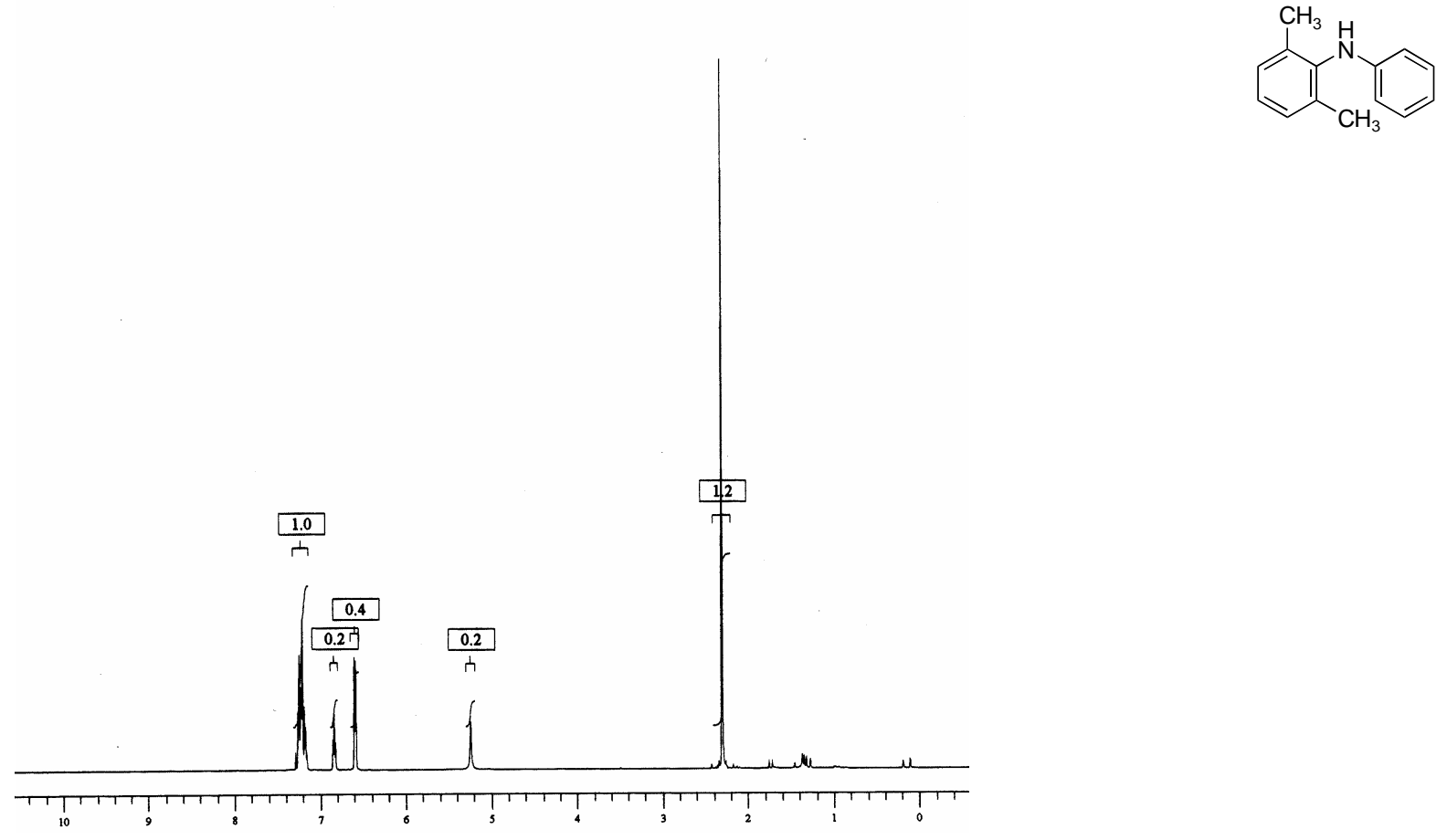

Figure S15. ${ }^{1} \mathrm{H}$ NMR spectrum $\left(\mathrm{CDCl}_{3}, 500 \mathrm{MHz}\right)$ of $\mathrm{N}$-(2,6-dimethylphenyl)aniline (Table 3, entry 8).

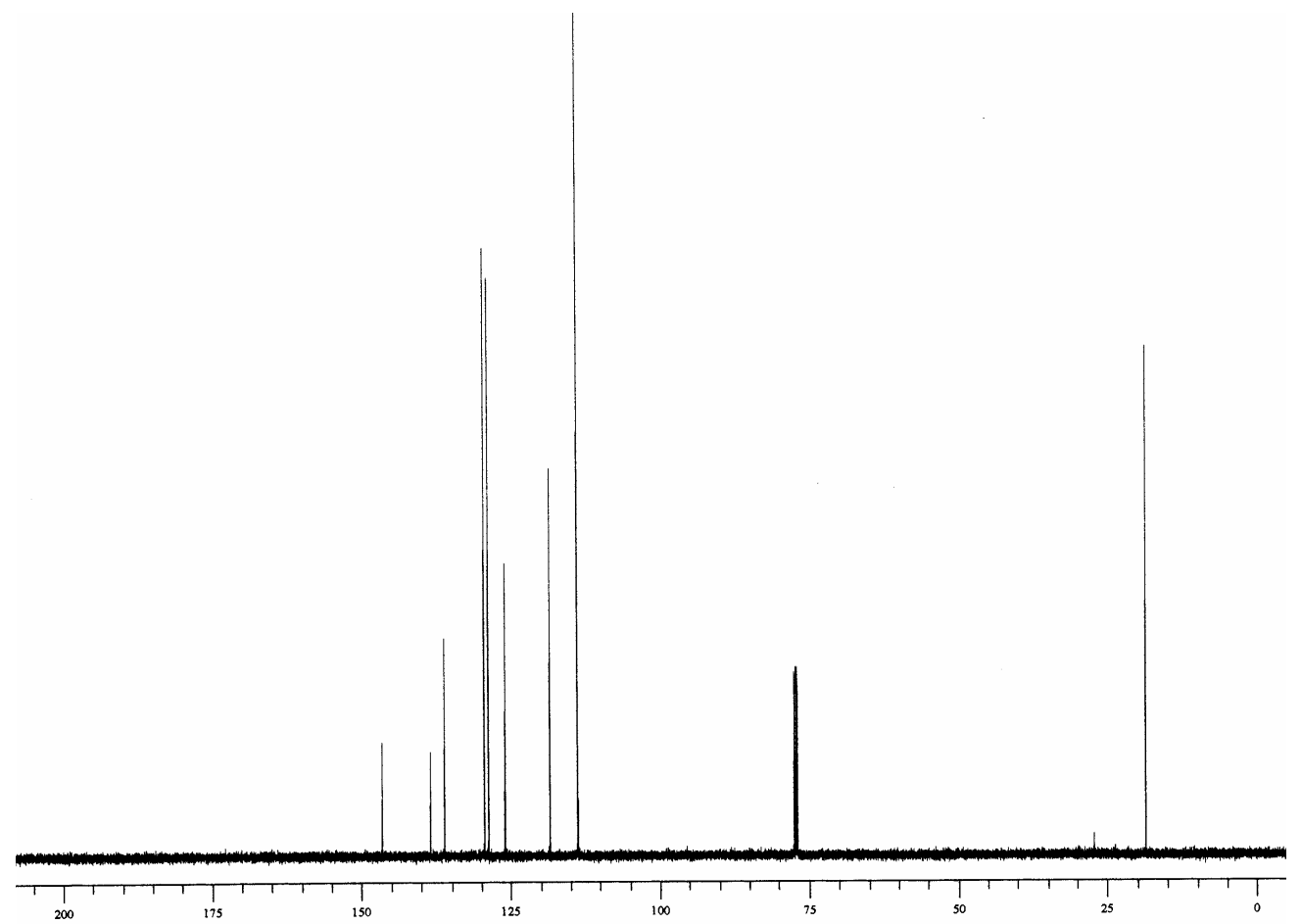

Figure S16. ${ }^{13} \mathrm{C}$ NMR spectrum $\left(\mathrm{CDCl}_{3}, 90.6 \mathrm{MHz}\right)$ of $\mathrm{N}$-(2,6-dimethylphenyl)aniline (Table 3, entry 8). 

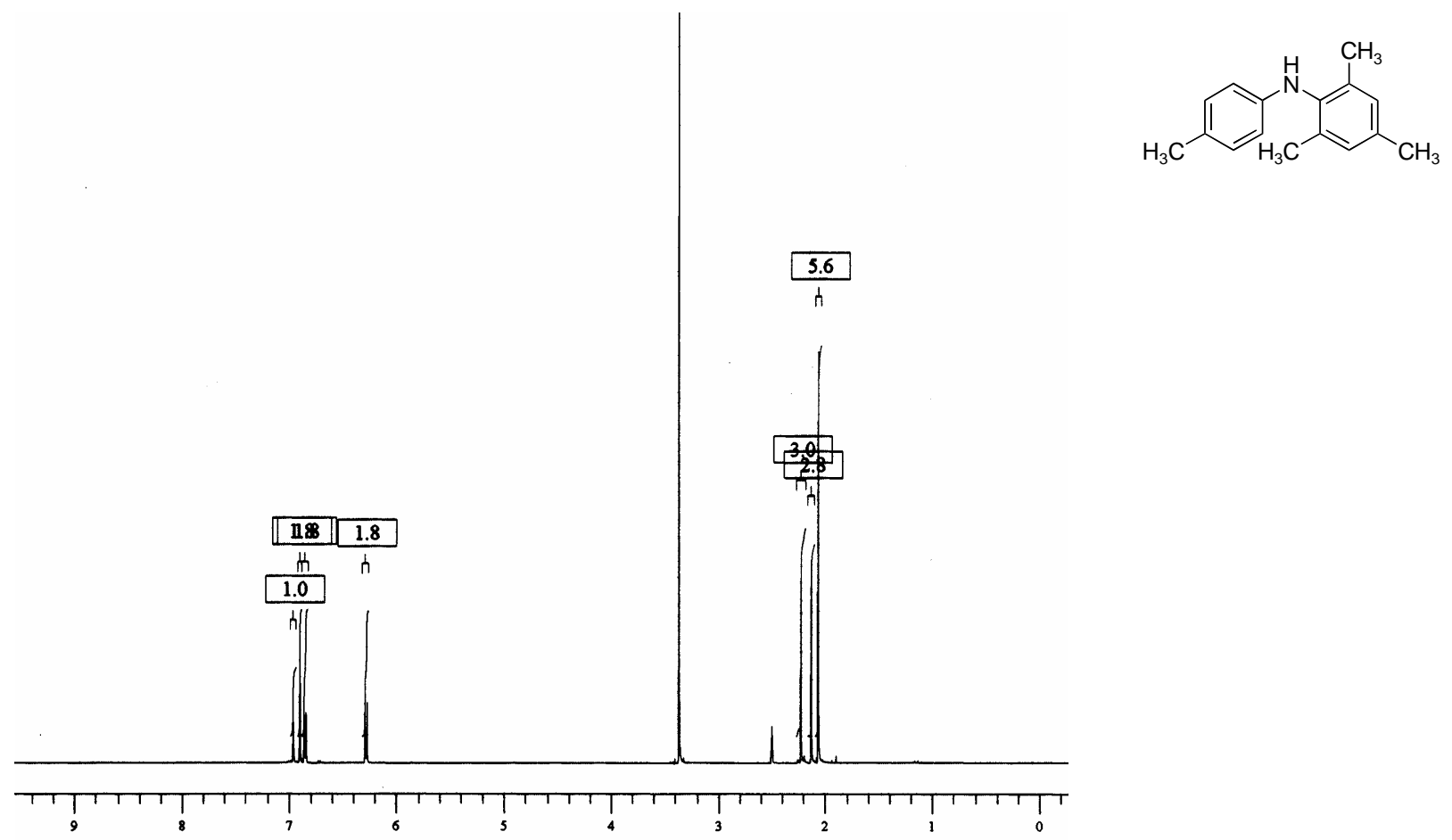

Figure S17. ${ }^{1} \mathrm{H}$ NMR spectrum (DMSO- $d_{6}, 500 \mathrm{MHz}$ ) of 2.4.6-trimethyl- $N$-(4methylphenyl)aniline (Table 3, entry 9).

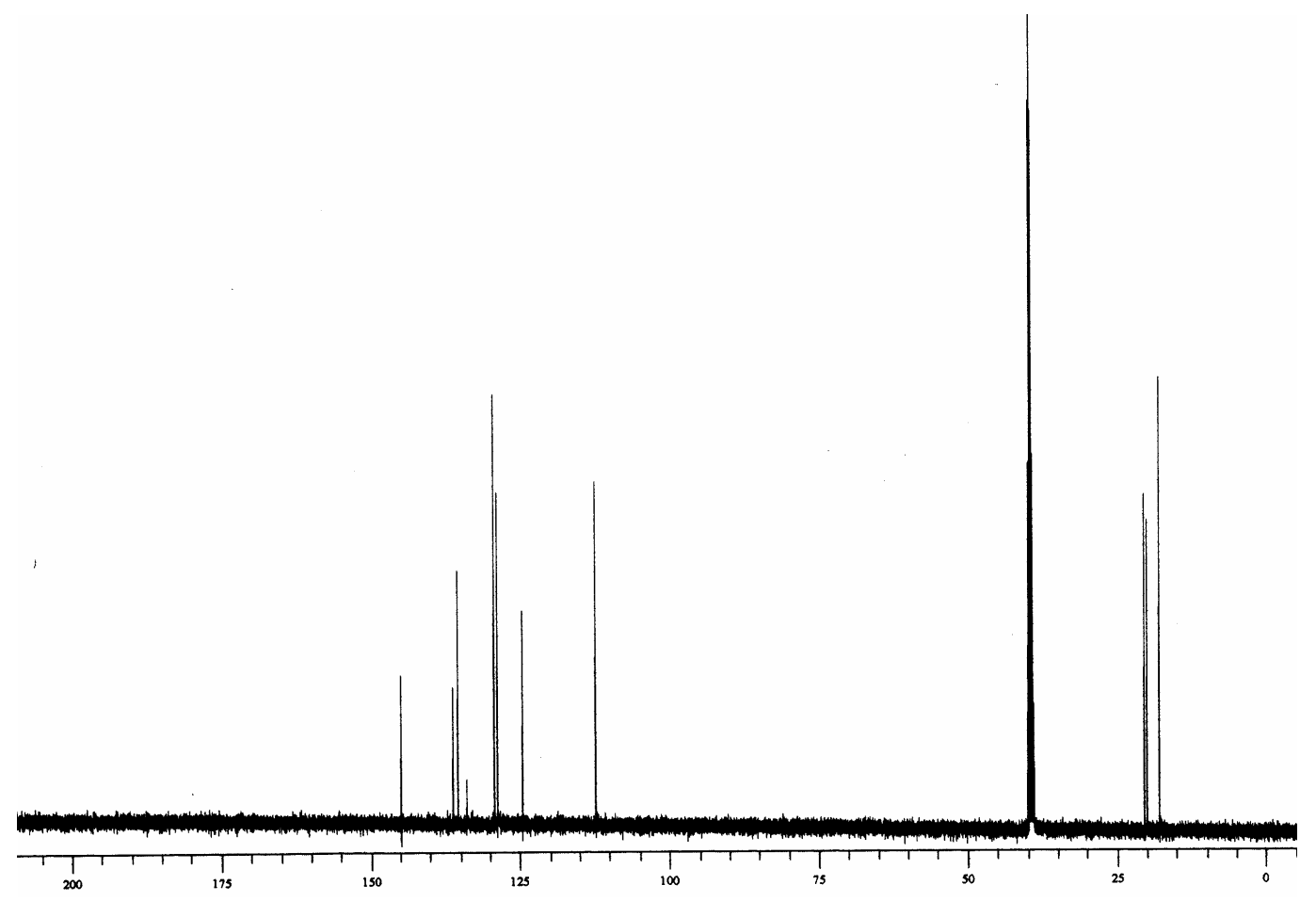

Figure S18. ${ }^{13} \mathrm{C}$ NMR spectrum (DMSO- $d_{6}, 90.6 \mathrm{MHz}$ ) of 2.4.6-trimethyl- $N$-(4methylphenyl)aniline (Table 3, entry 9). 


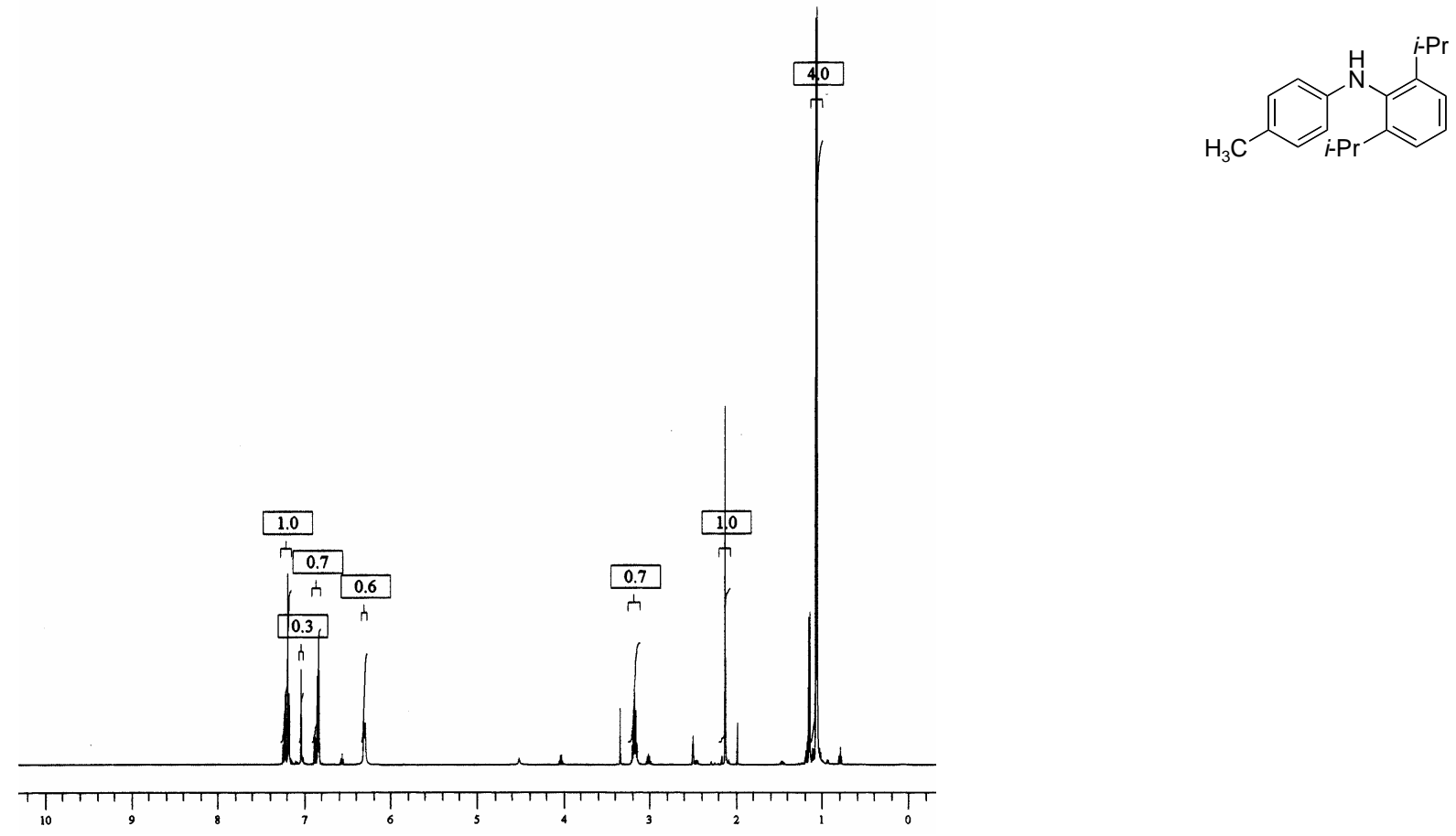

Figure S19. ${ }^{1} \mathrm{H}$ NMR spectrum (DMSO- $d_{6}, 500 \mathrm{MHz}$ ) of 2,6-diisopropyl- $N$-(4methylphenyl)aniline (Table 3 , entry 10).

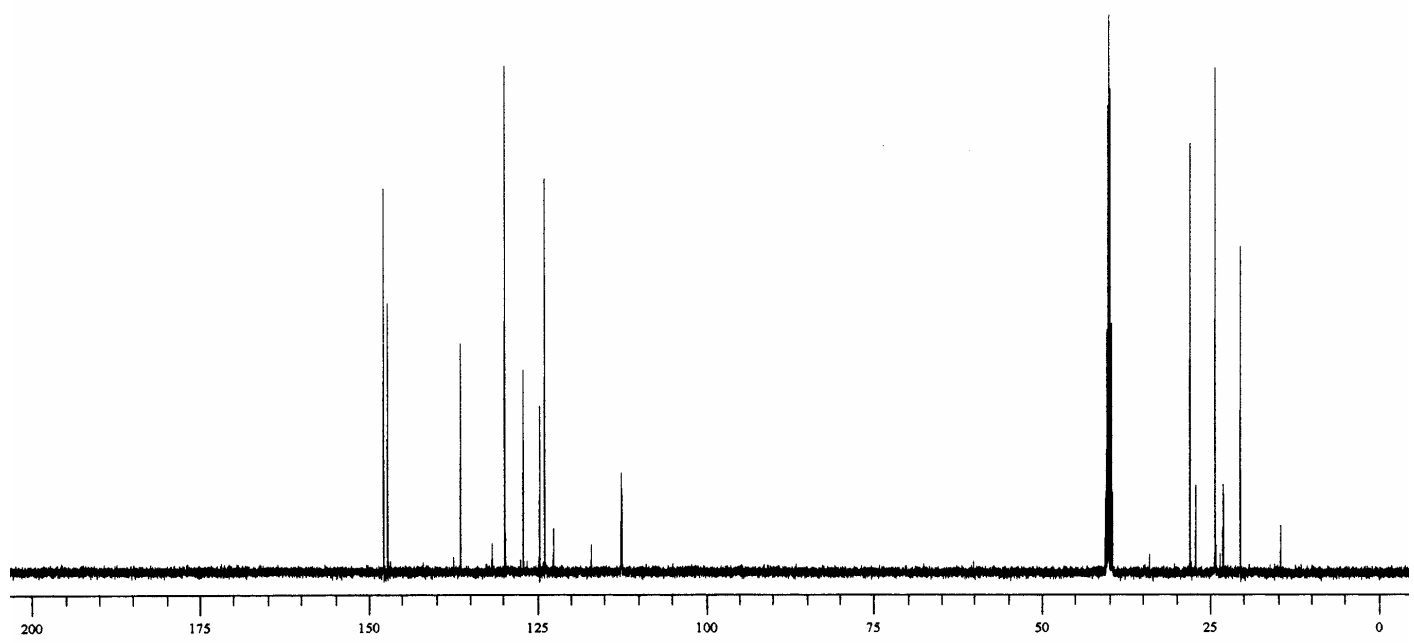

Figure S20. ${ }^{13} \mathrm{C}$ NMR spectrum (DMSO- $d_{6}, 90.6 \mathrm{MHz}$ ) of 2,6-diisopropyl- $\mathrm{N}$-(4methylphenyl)aniline (Table 3, entry 10). 


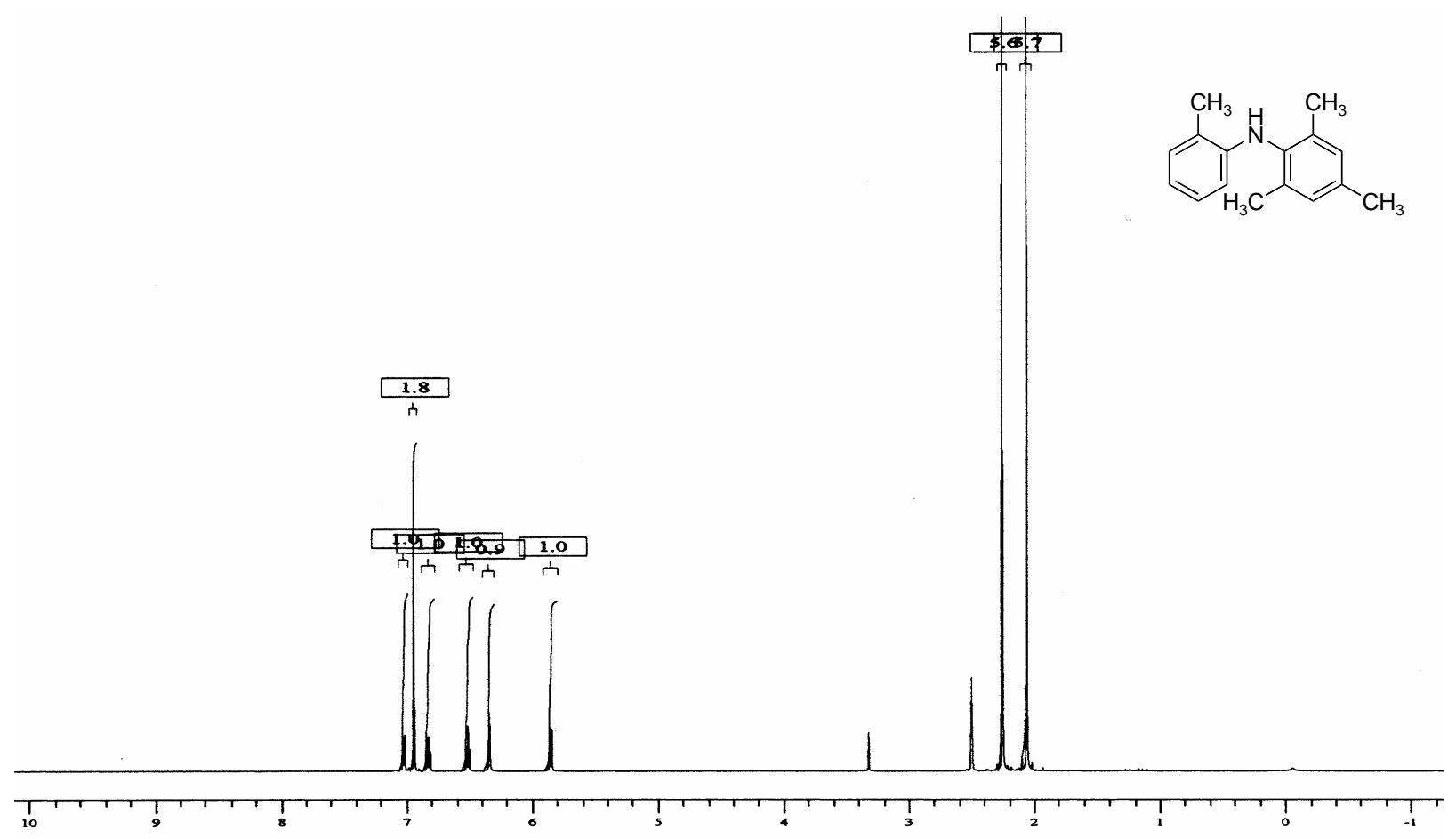

Figure S21. ${ }^{1} \mathrm{H}$ NMR spectrum $\left(\mathrm{CDCl}_{3}, 500 \mathrm{MHz}\right)$ of 2,4,6-trimethyl- $N$-(2-methylphenyl)aniline (Table 3, entry 11).

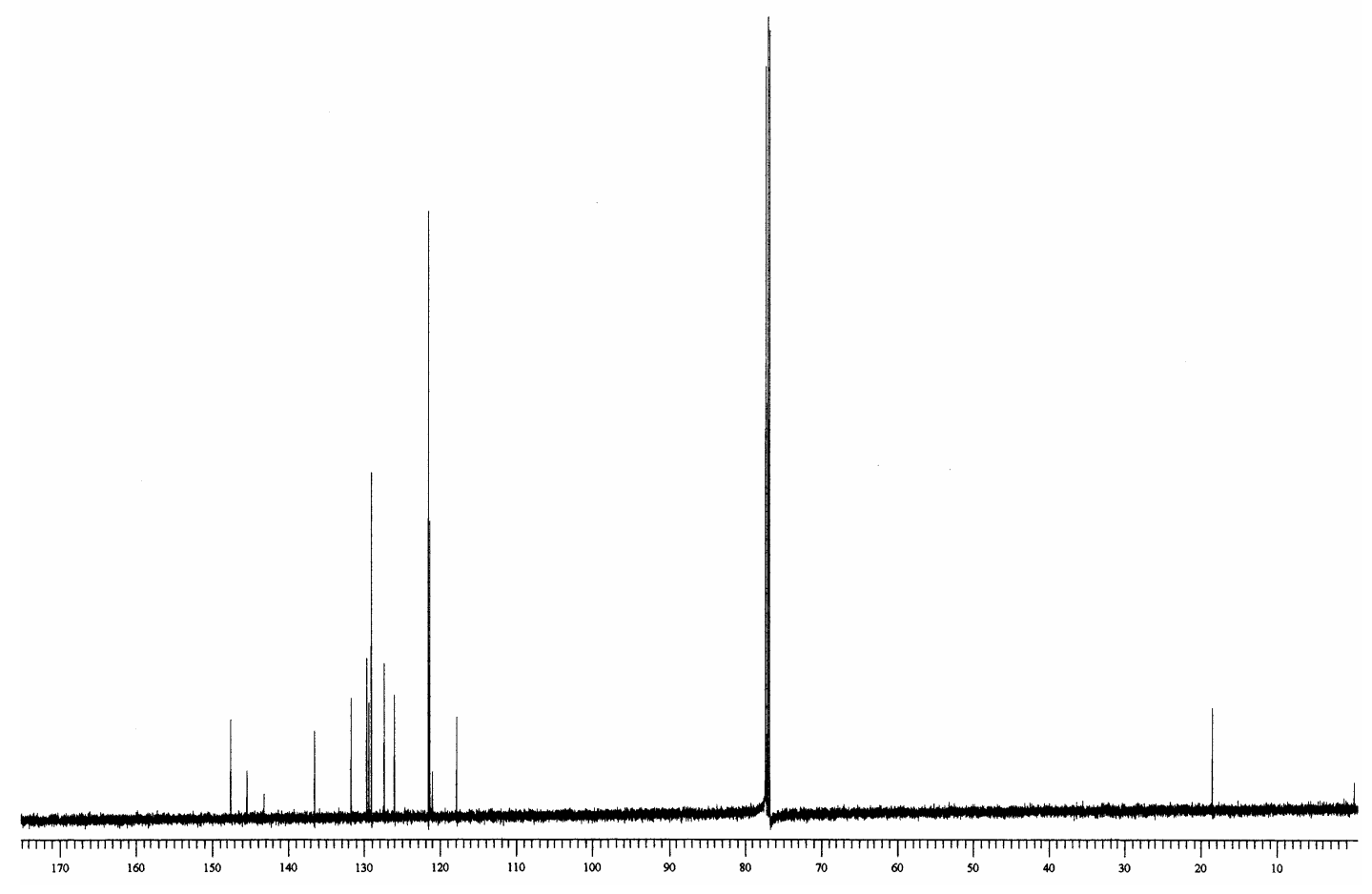

Figure S22. ${ }^{13} \mathrm{C}$ NMR spectrum $\left(\mathrm{CDCl}_{3}, 90.6 \mathrm{MHz}\right)$ of 2,4,6-trimethyl- $\mathrm{N}-(2-$ methylphenyl)aniline (Table 3 , entry 11). 


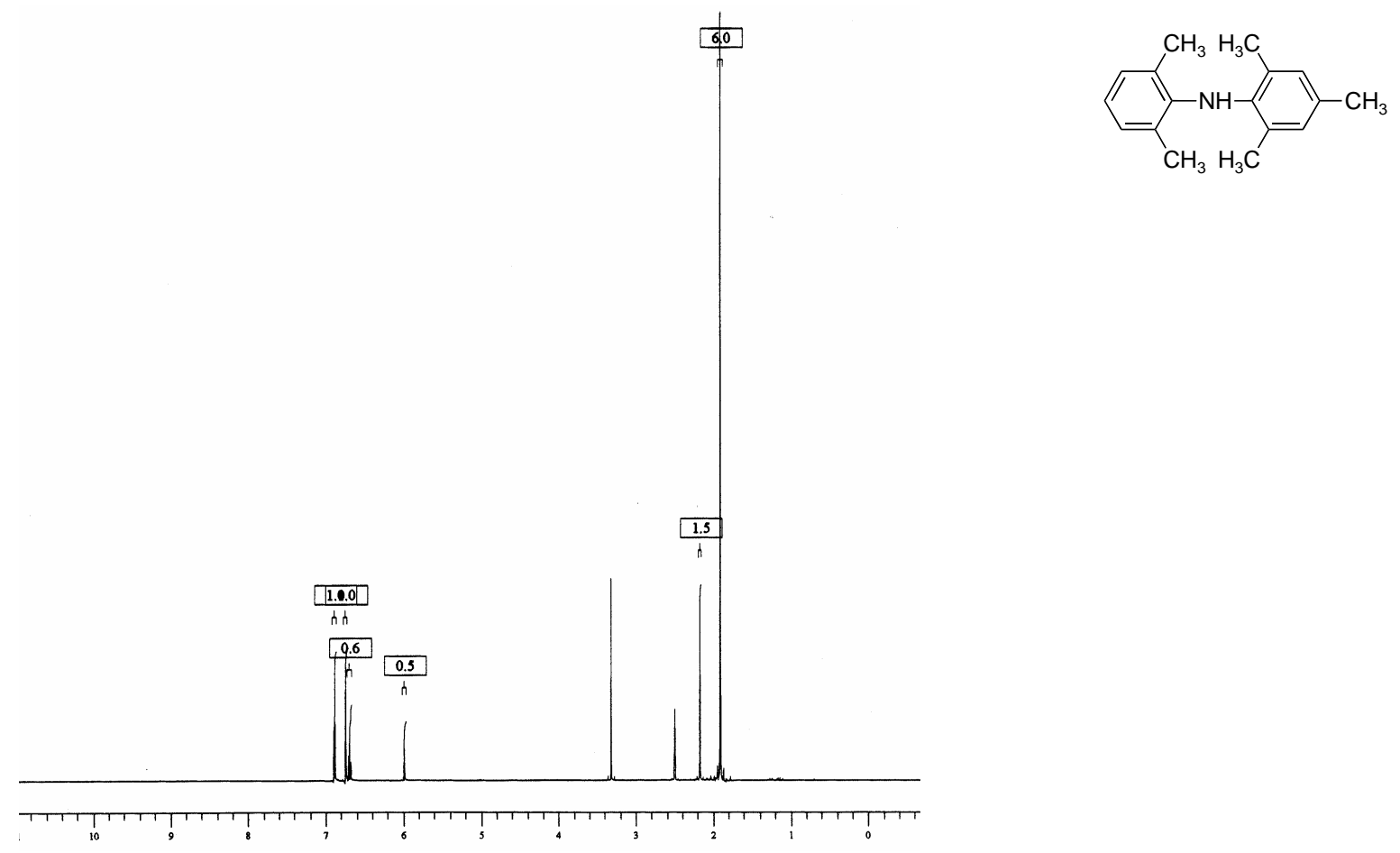

Figure S23. ${ }^{1} \mathrm{H}$ NMR spectrum (DMSO- $d_{6}, 500 \mathrm{MHz}$ ) of 2,4,6-trimethyl- $N$-(2,6dimethylphenyl)aniline (Table 3, entry 12).

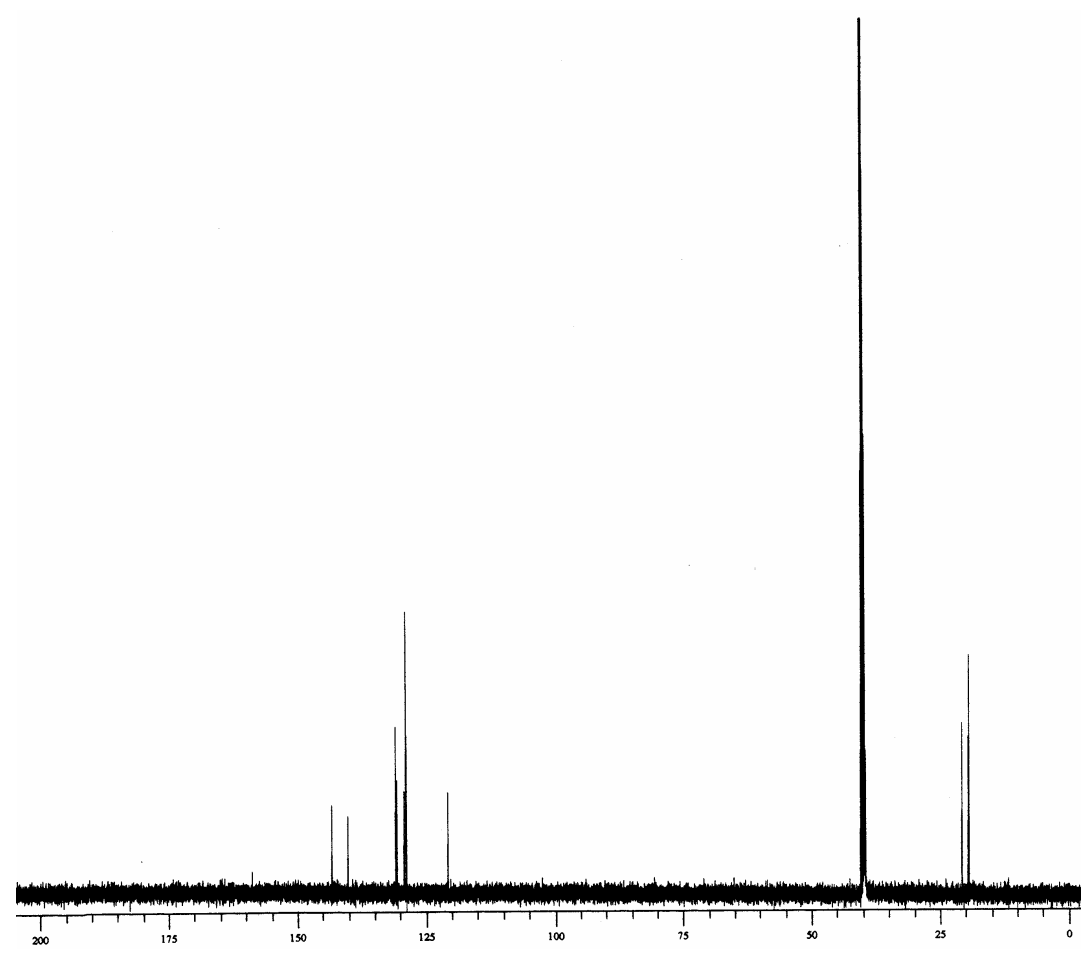

Figure S24. ${ }^{13} \mathrm{C}$ NMR spectrum (DMSO- $d_{6}, 90.6 \mathrm{MHz}$ ) of 2,4,6-trimethyl- $N$-(2,6dimethylphenyl)aniline (Table 3, entry 12). 

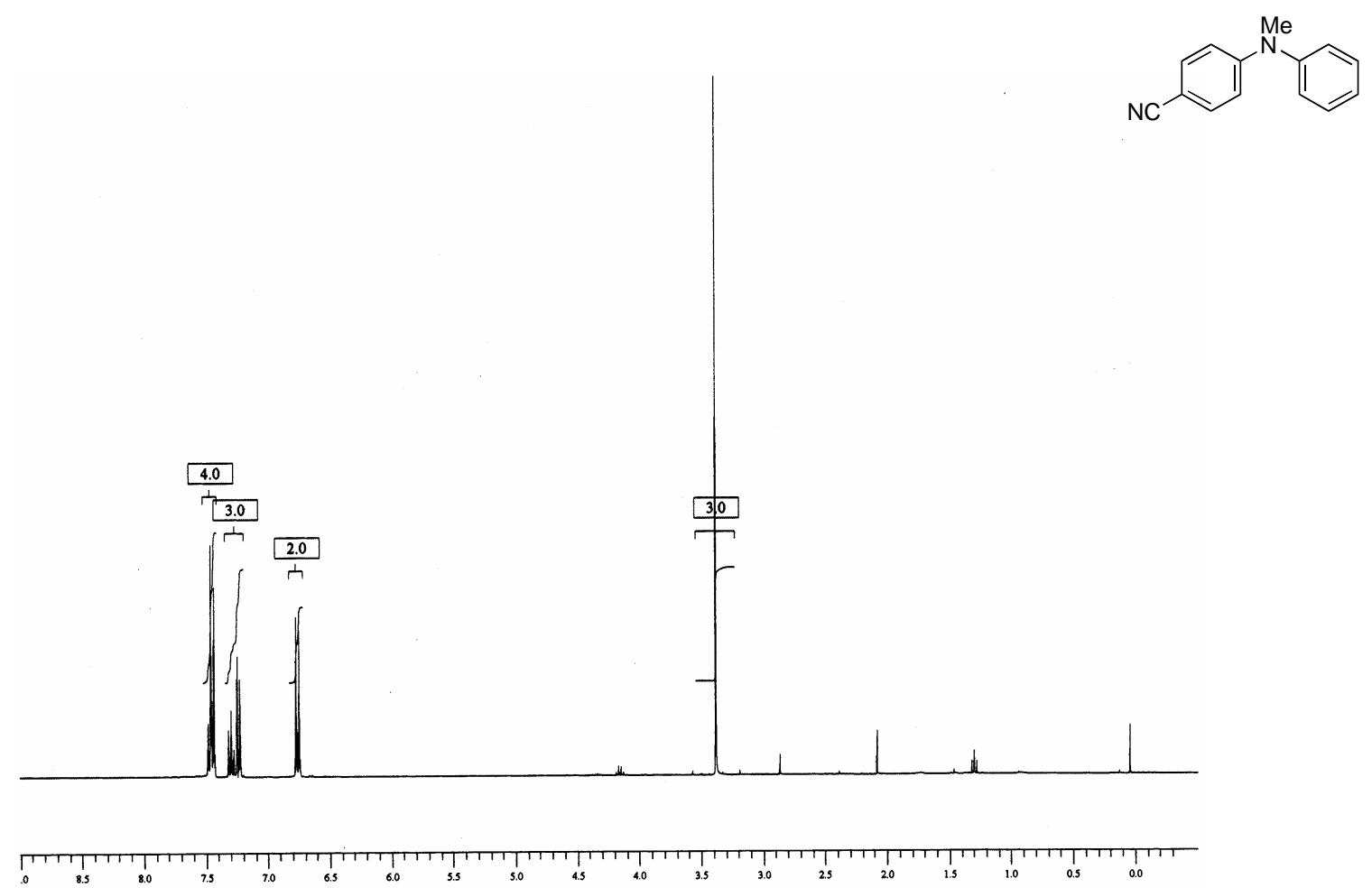

Figure S25. ${ }^{1} \mathrm{H}$ NMR spectrum $\left(\mathrm{CDCl}_{3}, 360 \mathrm{MHz}\right)$ of 4-( $N$-methyl- $N$-phenylamino)benzonitrile (Table 4, entry 1).

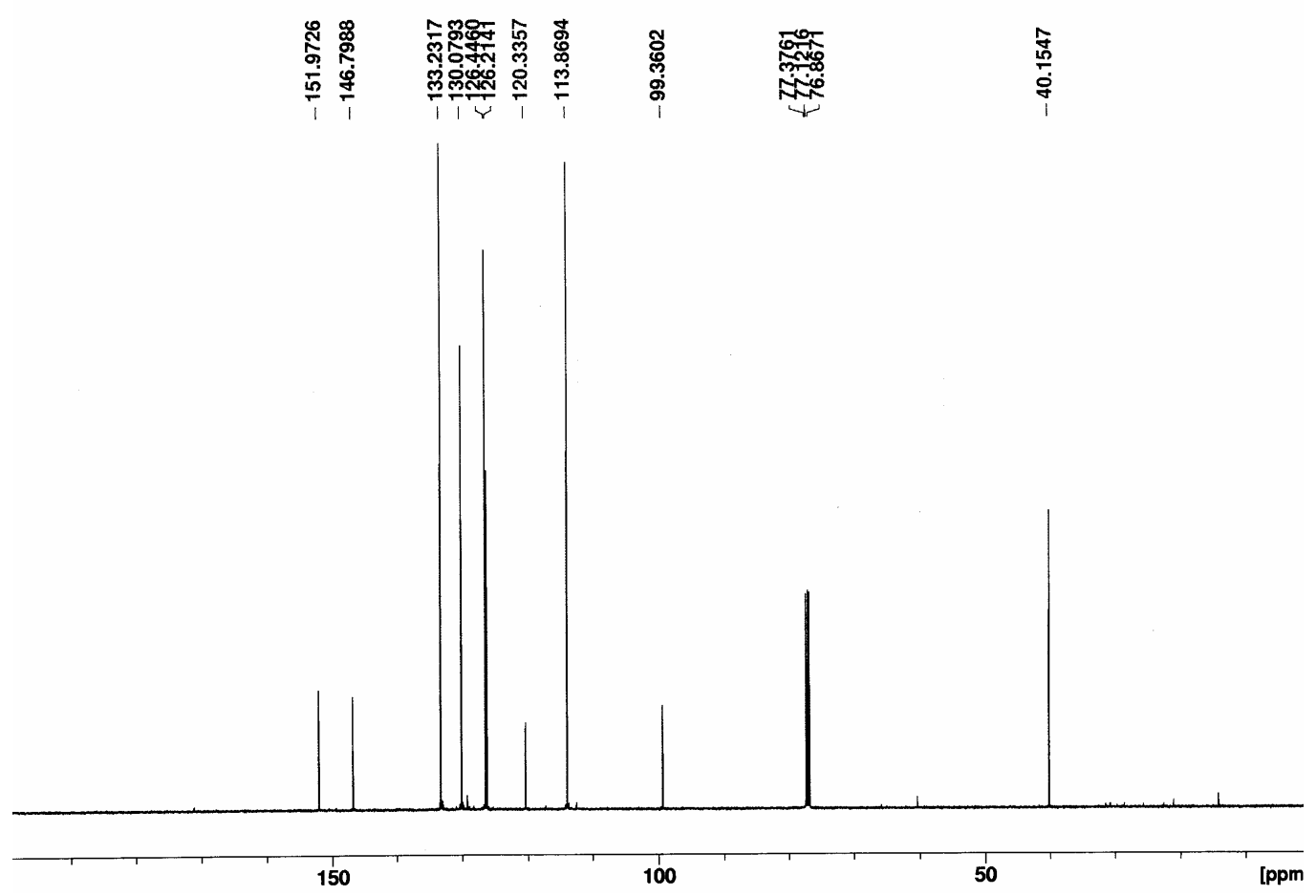

Figure S26. ${ }^{13} \mathrm{C}$ NMR spectrum $\left(\mathrm{CDCl}_{3}, 90.6 \mathrm{MHz}\right)$ of $4-(N$-methyl- $N$ phenylamino)benzonitrile (Table 4, entry 1). 


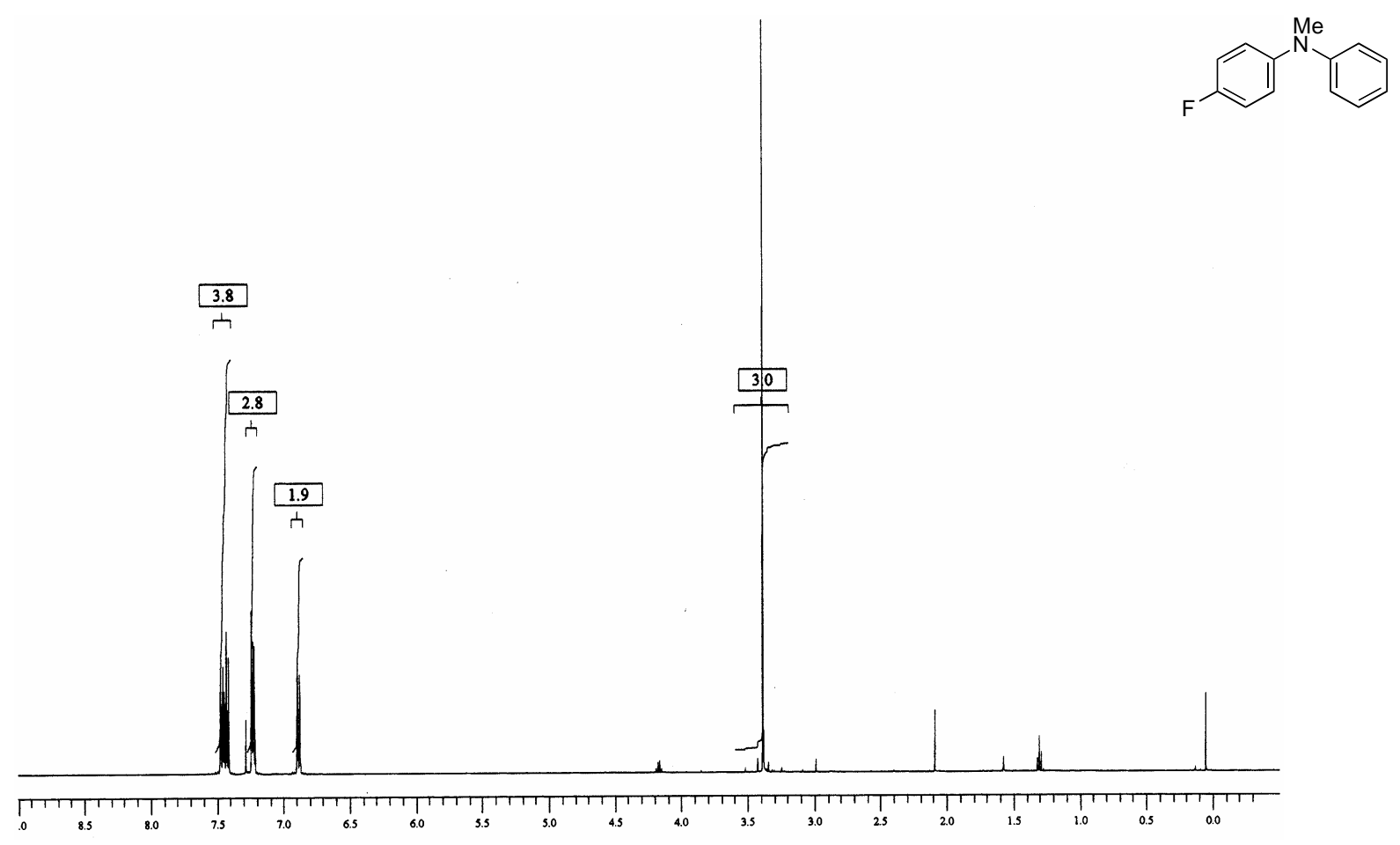

Figure S27. ${ }^{1} \mathrm{H}$ NMR spectrum $\left(\mathrm{CDCl}_{3}, 360 \mathrm{MHz}\right)$ of $N$-(4-fluorophenyl)- $N$-methylaniline (Table 4, entry 2).

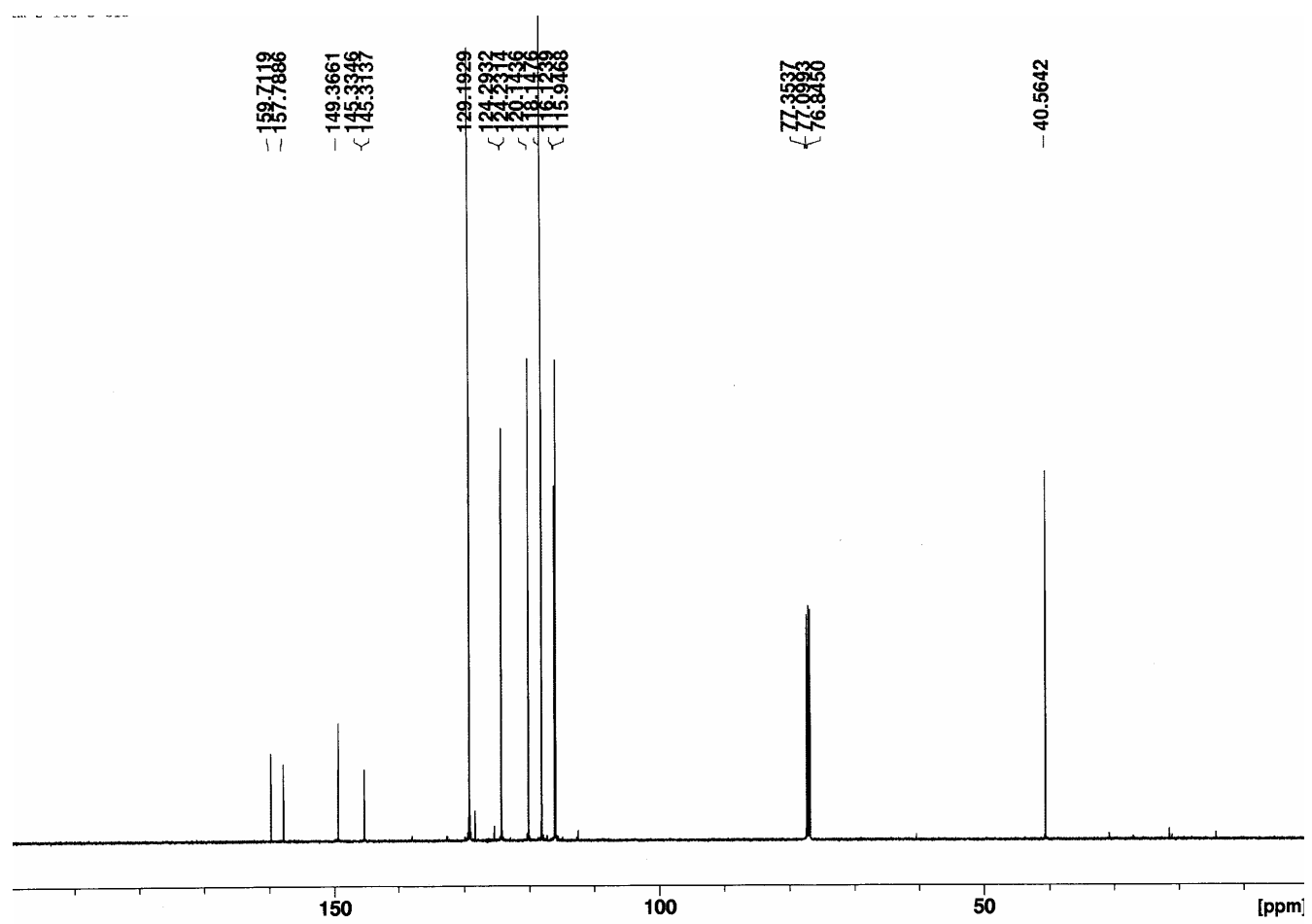

Figure S28. ${ }^{13} \mathrm{C}$ NMR spectrum $\left(\mathrm{CDCl}_{3}, 90.6 \mathrm{MHz}\right)$ of $N$-(4-fluorophenyl)- $N$-methylaniline (Table 4, entry 2). 


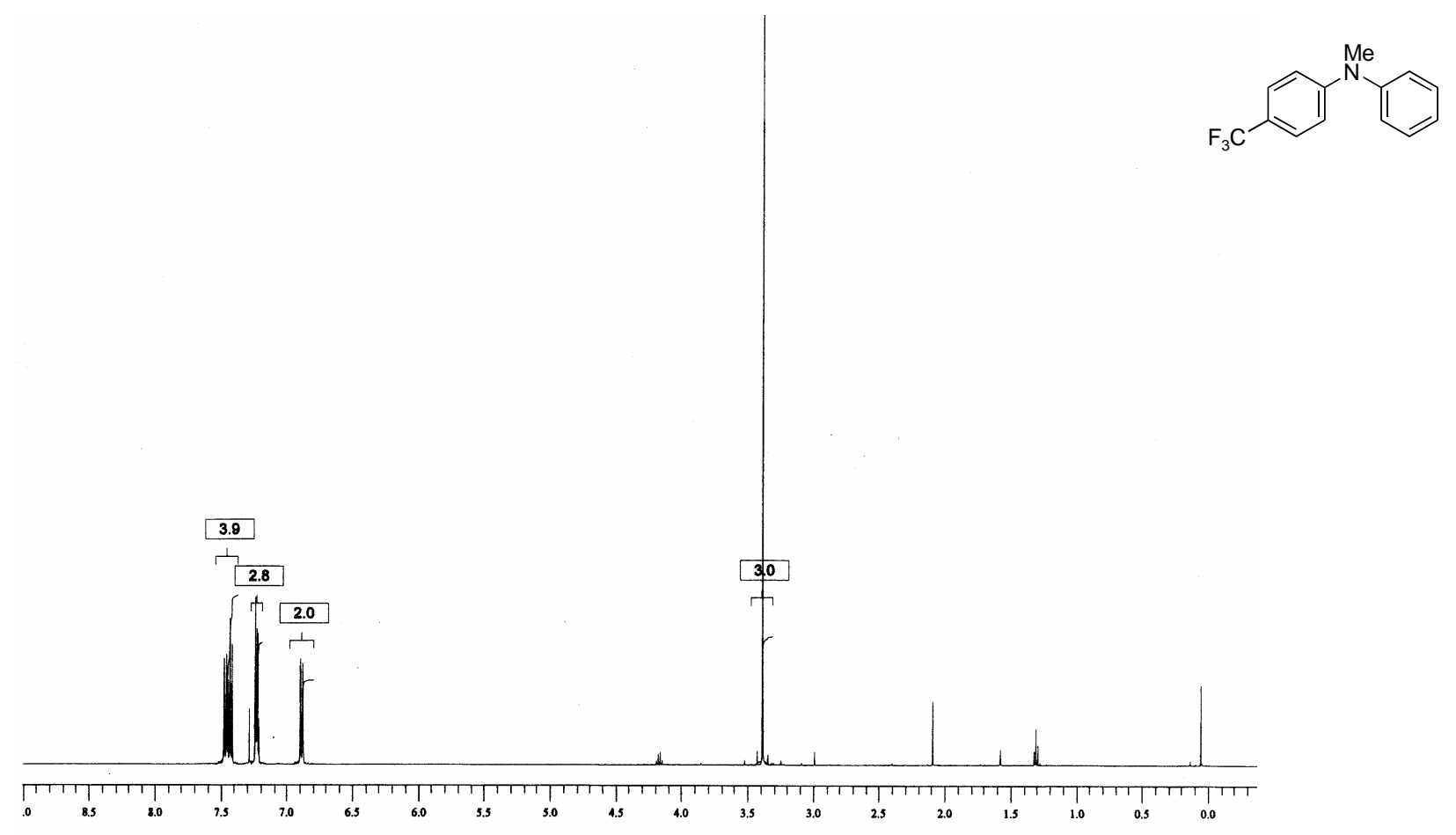

Figure S29. ${ }^{1} \mathrm{H}$ NMR spectrum $\left(\mathrm{CDCl}_{3}, 360 \mathrm{MHz}\right)$ of $N$-(4-trifluoromethylphenyl)- $N$ methylaniline (Table 4, entry 3).

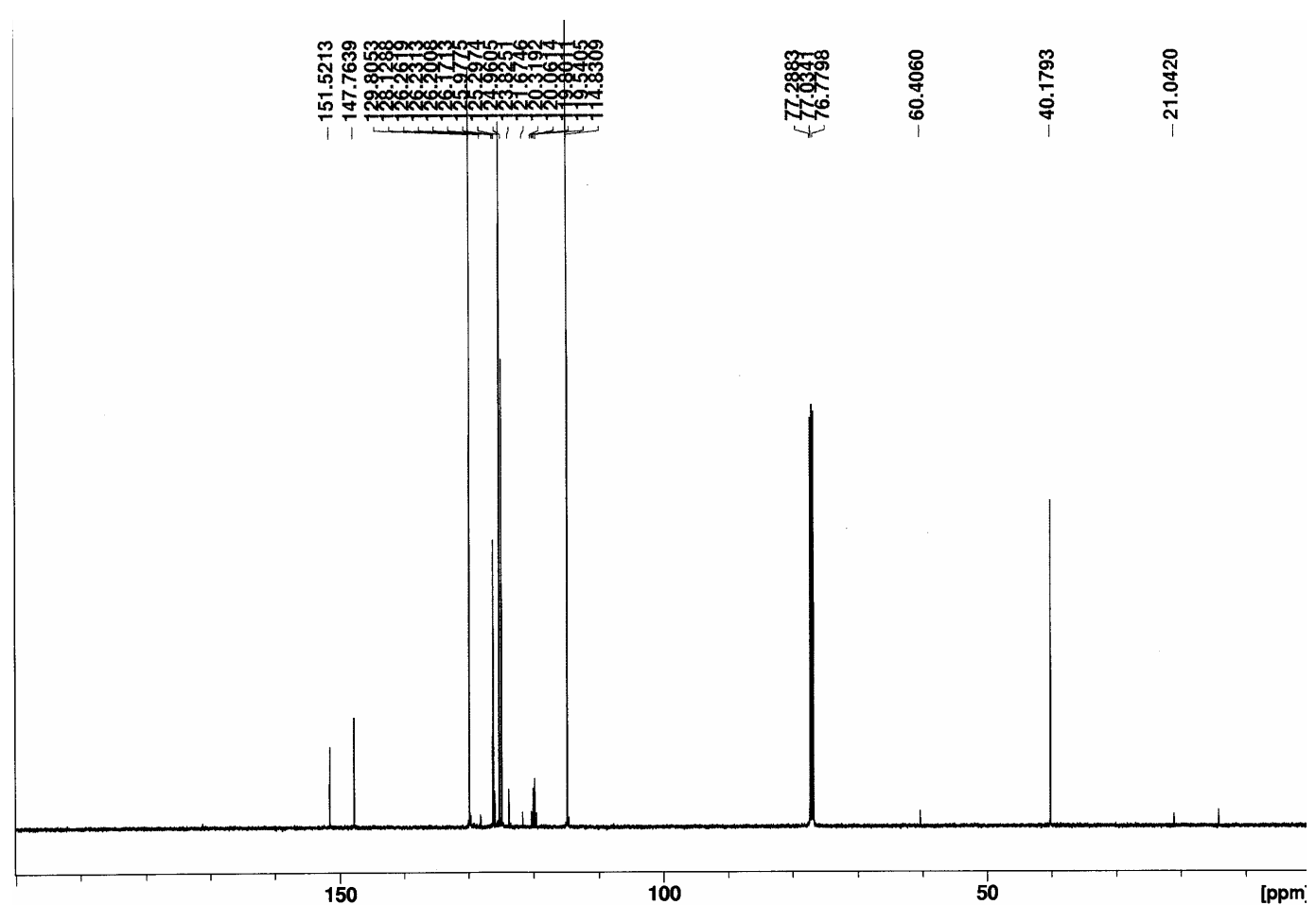

Figure S30. ${ }^{13} \mathrm{C}$ NMR spectrum $\left(\mathrm{CDCl}_{3}, 90.6 \mathrm{MHz}\right)$ of $N$-(4-trifluoromethylphenyl)- $N$ methylaniline (Table 4, entry 3 ). 


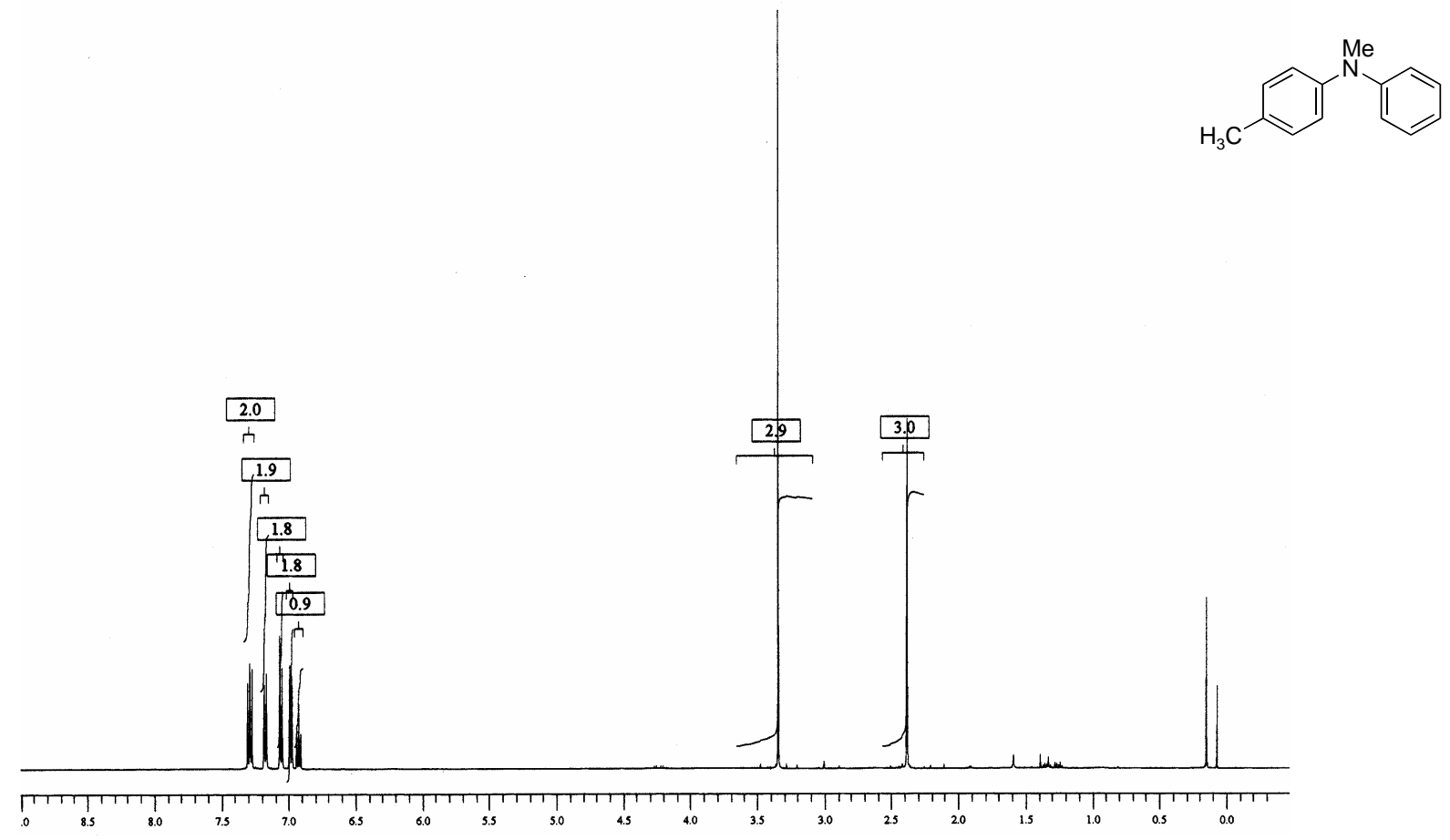

Figure S31. ${ }^{1} \mathrm{H}$ NMR spectrum $\left(\mathrm{CDCl}_{3}, 360 \mathrm{MHz}\right)$ of $N$-methyl- $N$-phenyl-p-toluidine (Table 4, entry 4).

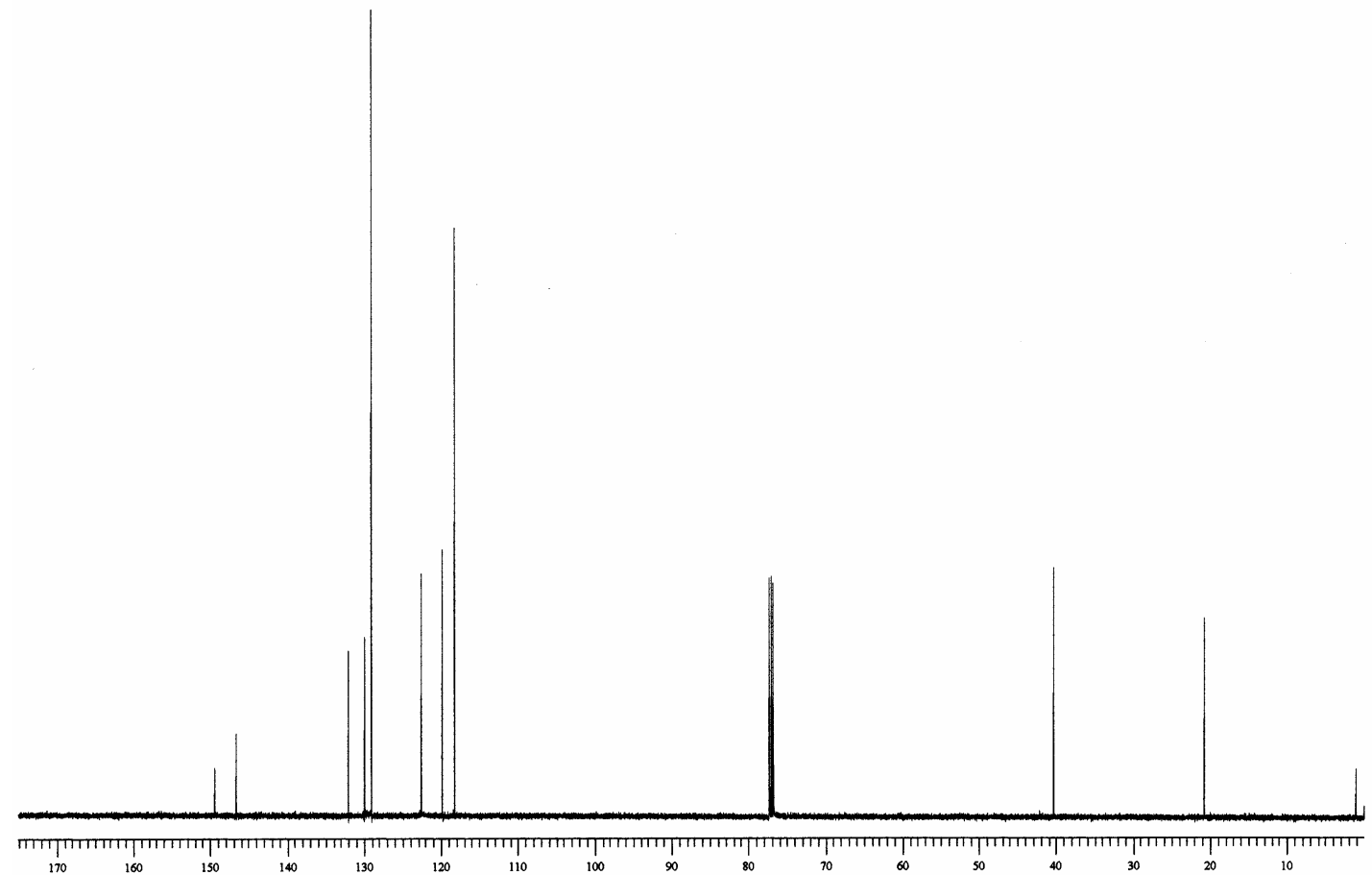

Figure S32. ${ }^{13} \mathrm{C}$ NMR spectrum $\left(\mathrm{CDCl}_{3}, 90.6 \mathrm{MHz}\right)$ of $N$-methyl- $N$-phenyl-p-toluidine (Table 4, entry 4). 


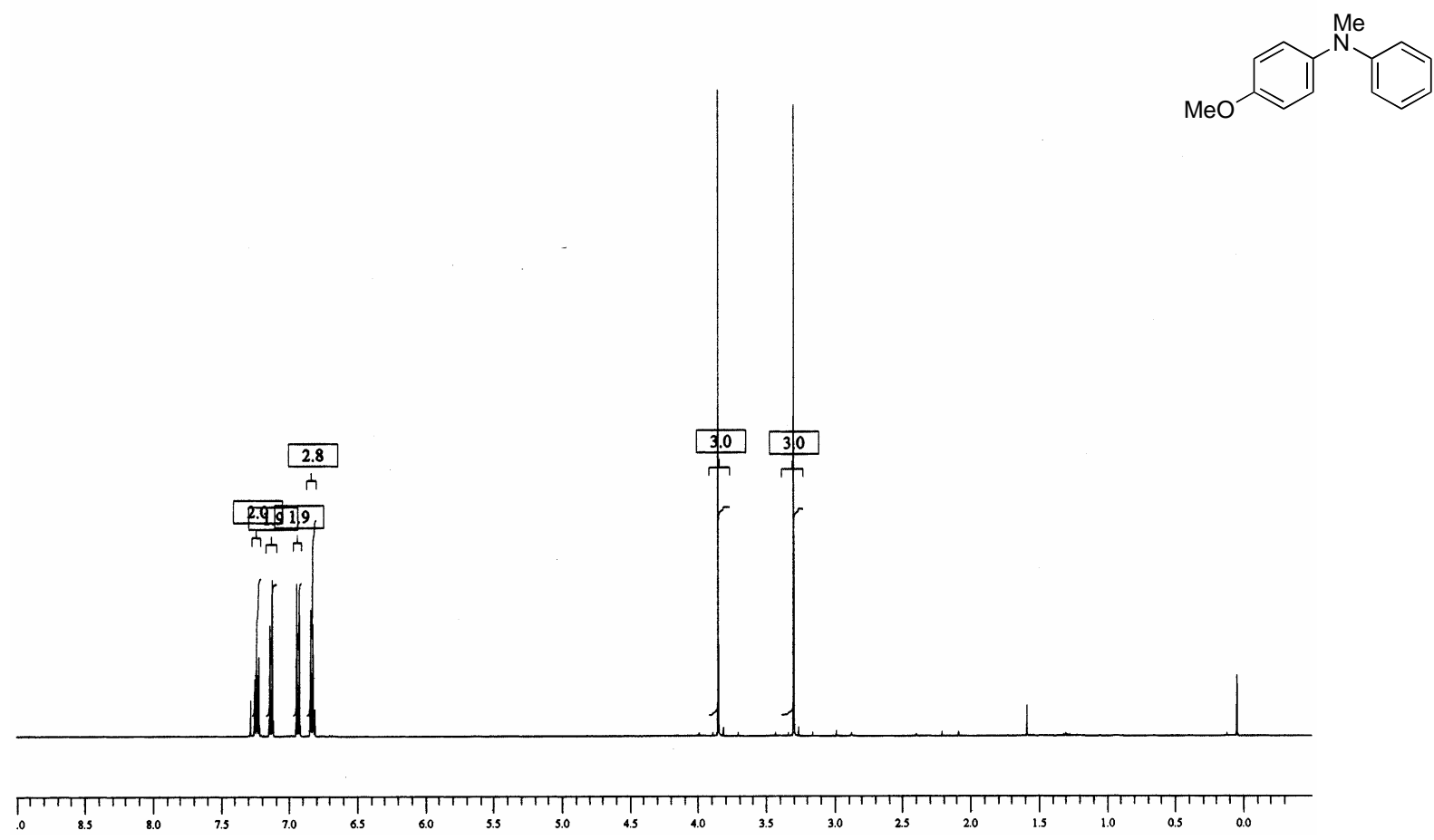

Figure S33. ${ }^{1} \mathrm{H}$ NMR spectrum $\left(\mathrm{CDCl}_{3}, 360 \mathrm{MHz}\right)$ of $N$-methyl- $N$-phenyl-p-anisidine (Table 4, entry 5).

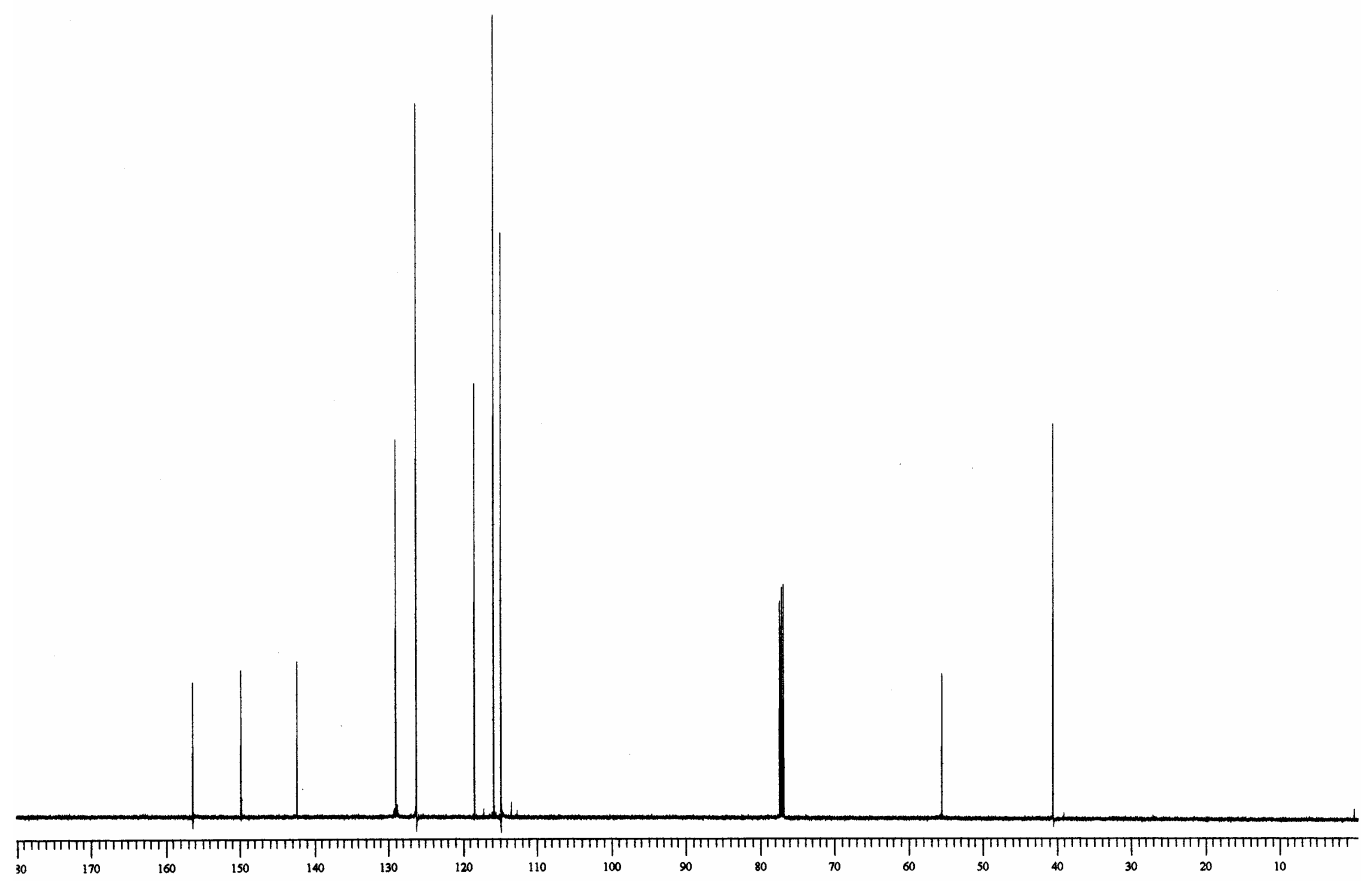

Figure S34. ${ }^{13} \mathrm{C}$ NMR spectrum $\left(\mathrm{CDCl}_{3}, 90.6 \mathrm{MHz}\right)$ of $N$-methyl- $N$-phenyl-p-anisidine (Table 4, entry 5). 


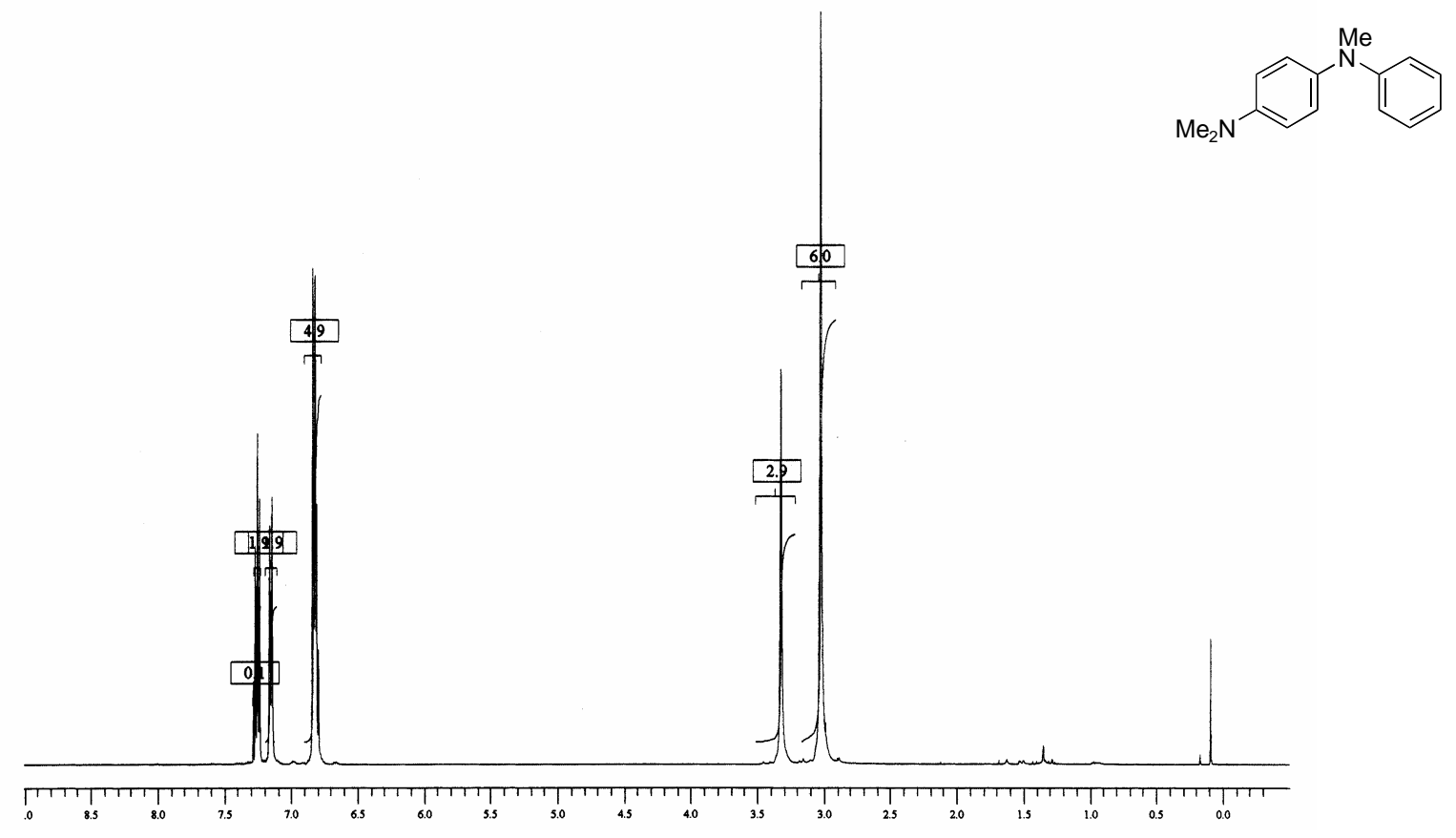

Figure S35. ${ }^{1} \mathrm{H}$ NMR spectrum $\left(\mathrm{CDCl}_{3}, 360 \mathrm{MHz}\right)$ of $N, N, N$ '-trimethyl- $N$ '-phenylbenzene-1,4diamine (Table 4, entry 6).

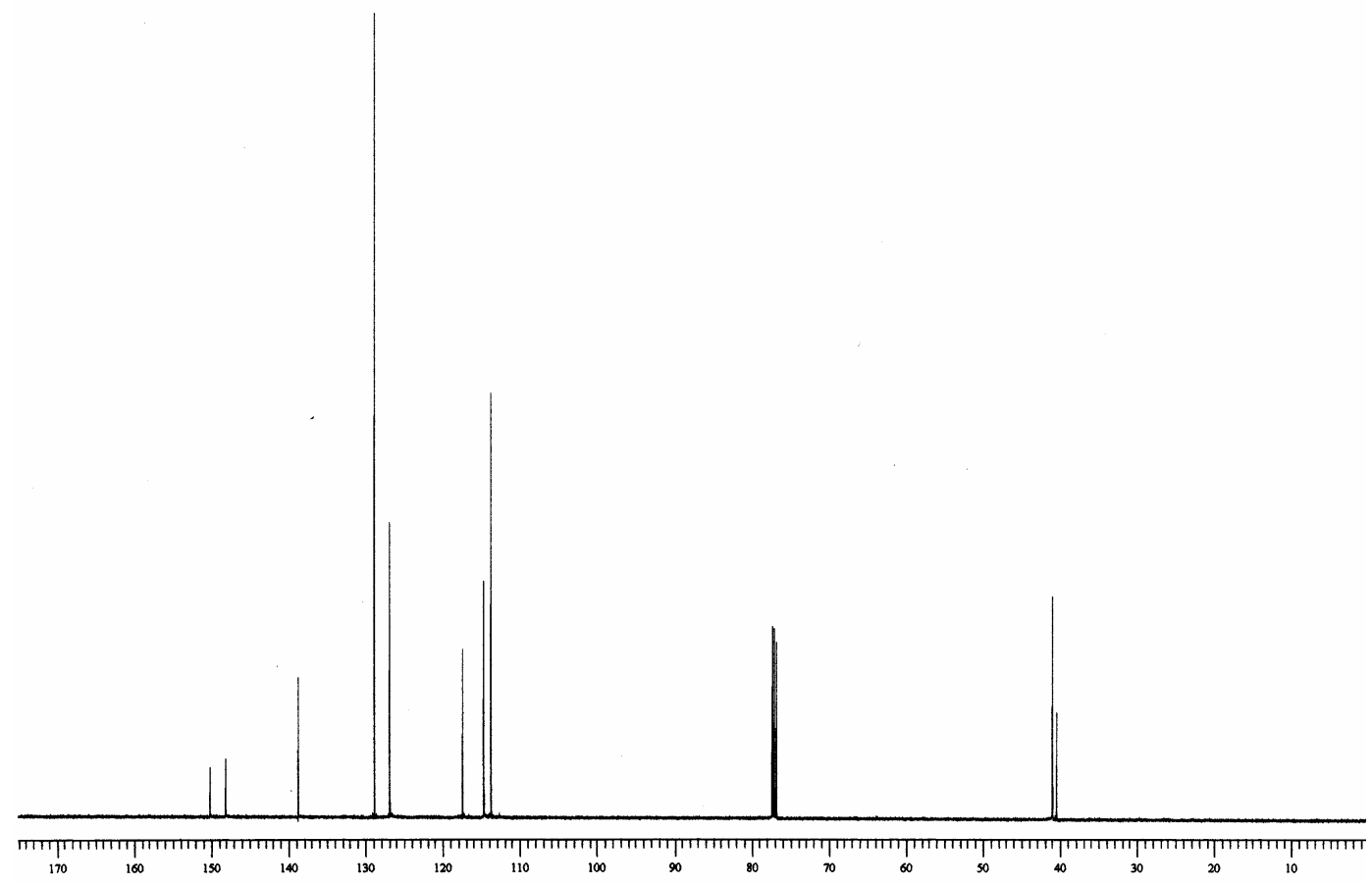

Figure S36. ${ }^{13} \mathrm{C}$ NMR spectrum $\left(\mathrm{CDCl}_{3}, 90.6 \mathrm{MHz}\right)$ of $N, N, N$ '-trimethyl- $N$ '-phenylbenzene-1,4diamine (Table 4, entry 6). 


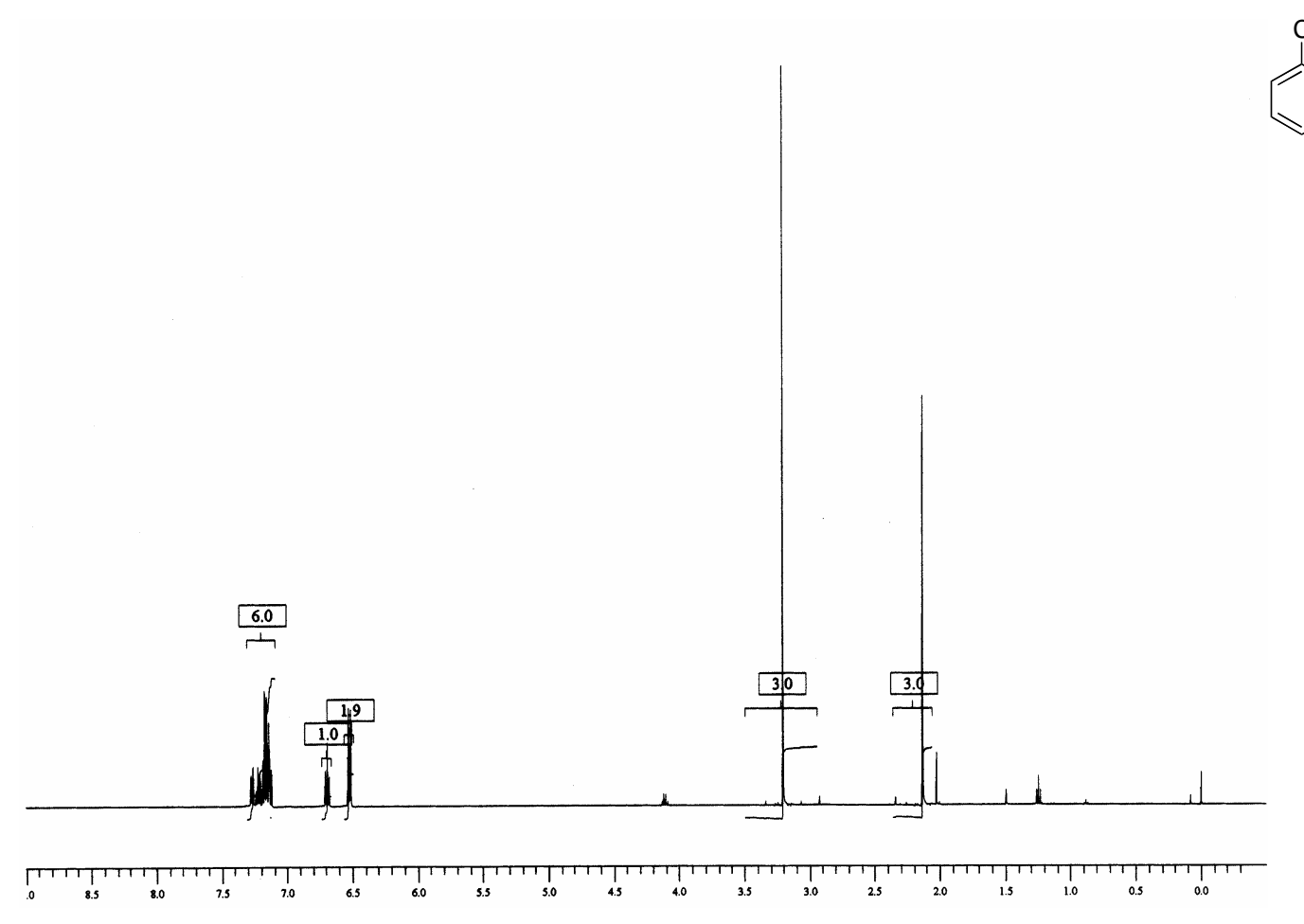

Figure S37. ${ }^{1} \mathrm{H}$ NMR spectrum $\left(\mathrm{CDCl}_{3}, 360 \mathrm{MHz}\right)$ of $N$-methyl- $N$-phenyl-o-toluidine (Table 4, entry 7).

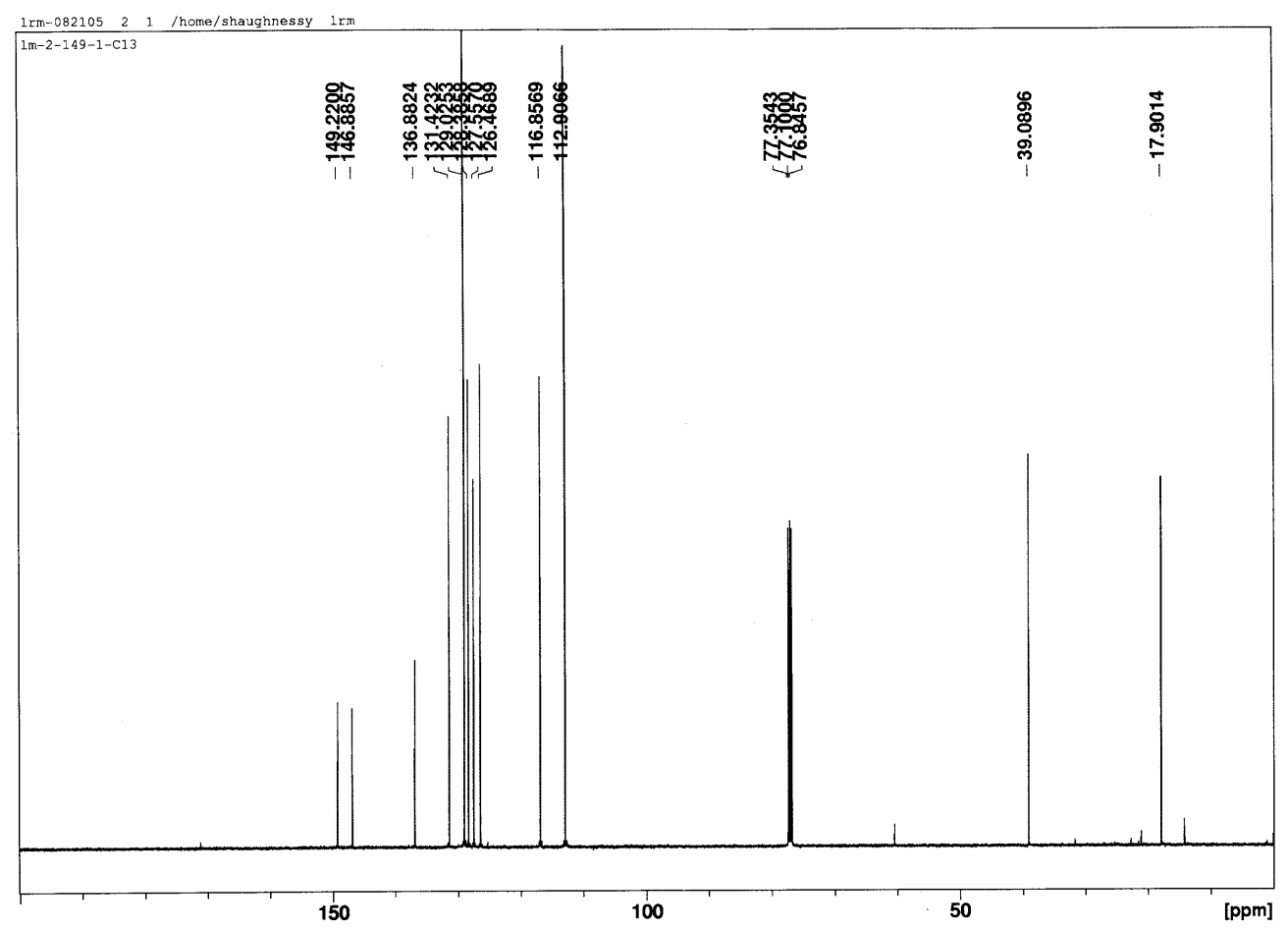

Figure S38. ${ }^{13} \mathrm{C}$ NMR spectrum $\left(\mathrm{CDCl}_{3}, 90.6 \mathrm{MHz}\right)$ of $N$-methyl- $N$-phenyl-o-toluidine (Table 4, entry 7). 


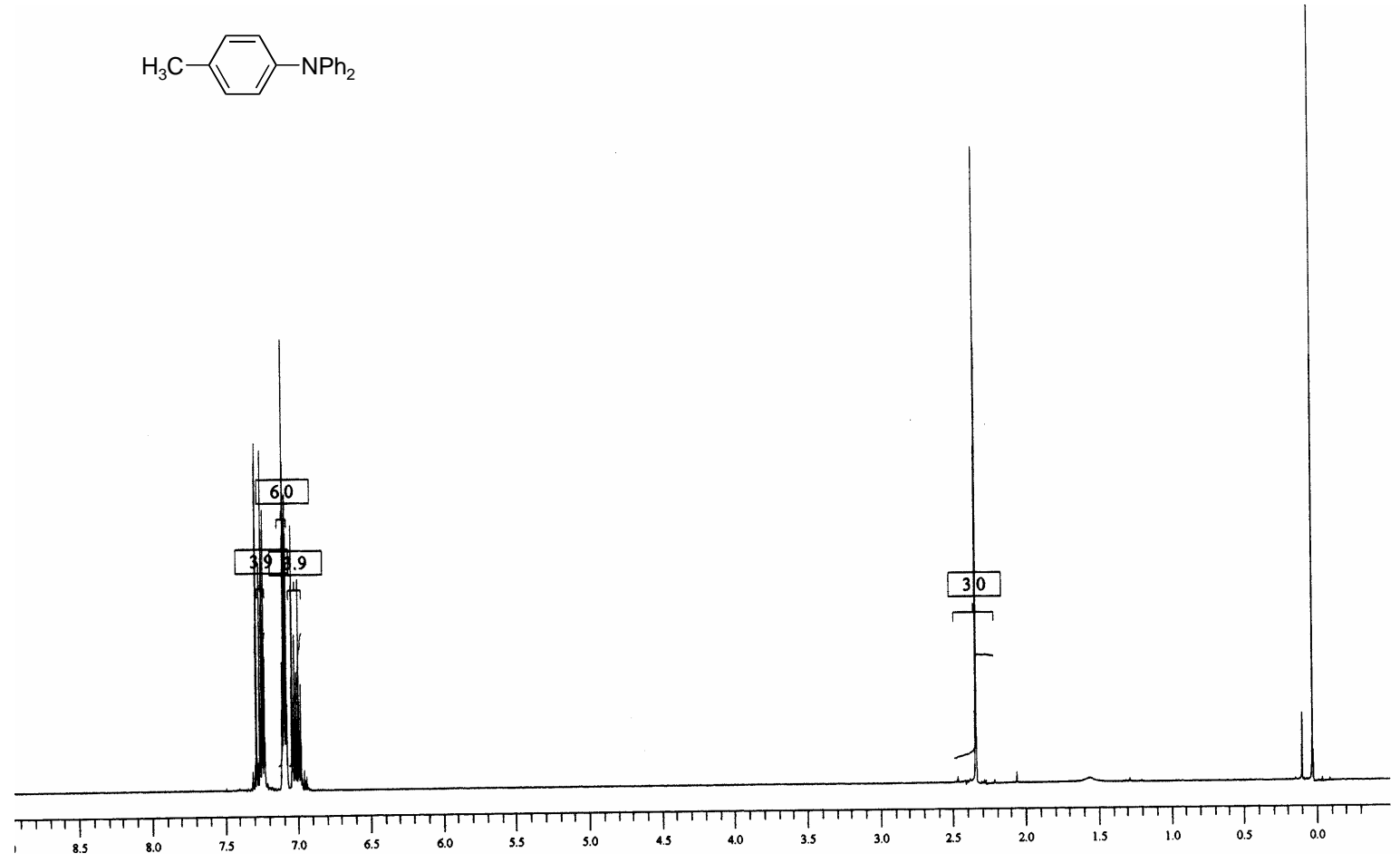

Figure S39. ${ }^{1} \mathrm{H}$ NMR spectrum $\left(\mathrm{CDCl}_{3}, 360 \mathrm{MHz}\right)$ of $N, N$-diphenyl-p-toluidine (Table 4, entry 9).

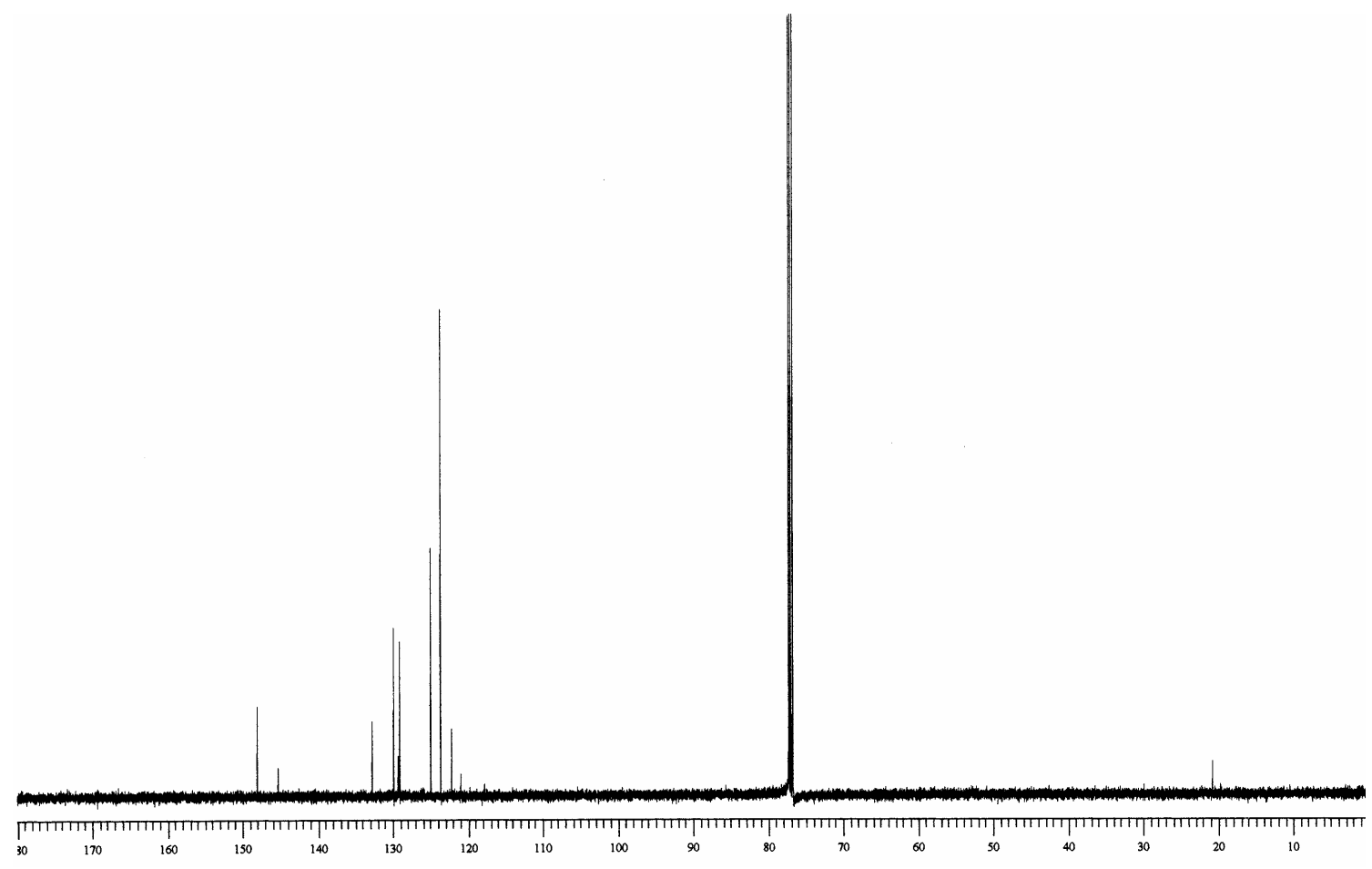

Figure S40. ${ }^{13} \mathrm{C}$ NMR spectrum $\left(\mathrm{CDCl}_{3}, 90.6 \mathrm{MHz}\right)$ of $N, N$-diphenyl-p-toluidine (Table 4, entry 9). 

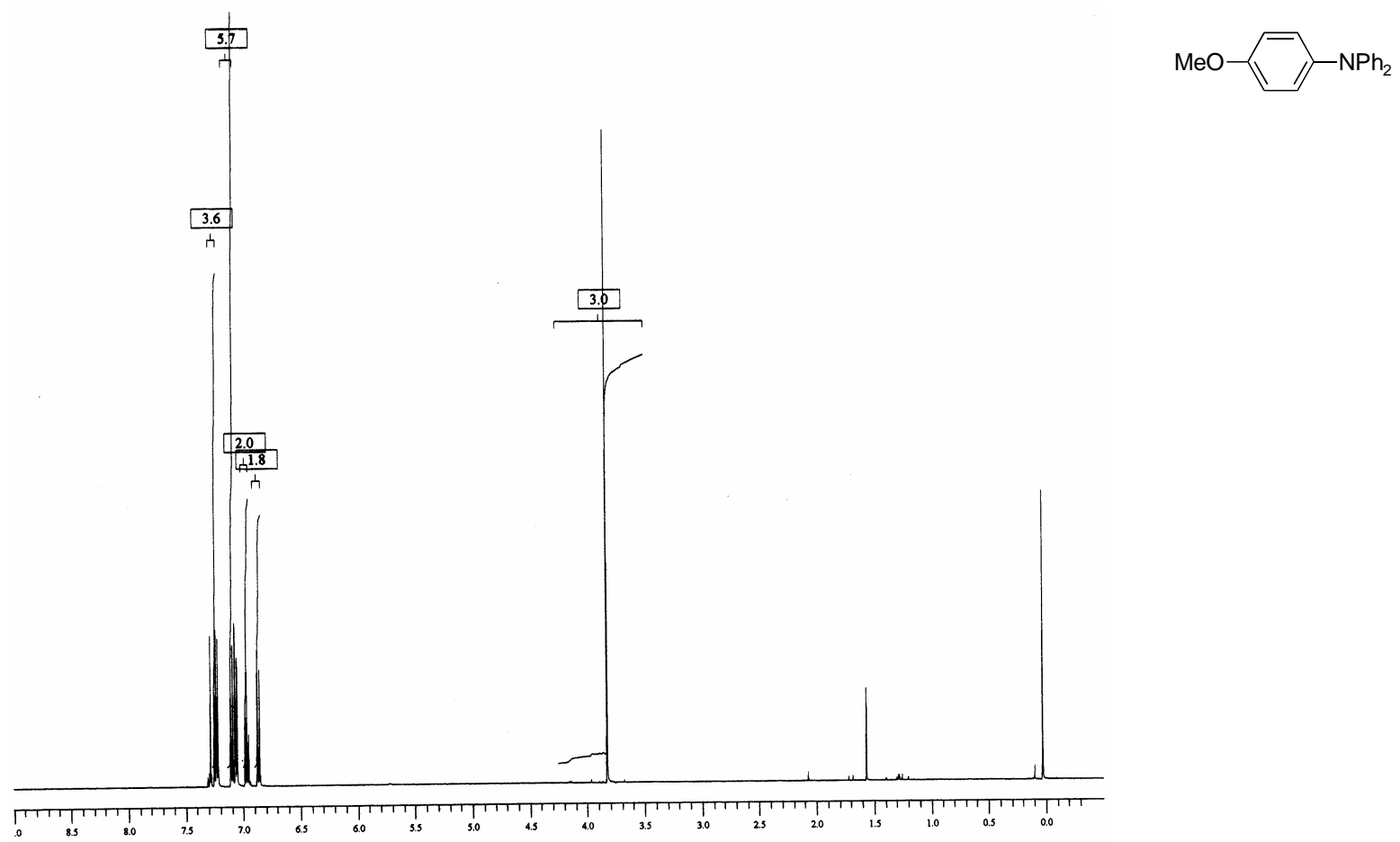

Figure S41. ${ }^{1} \mathrm{H}$ NMR spectrum $\left(\mathrm{CDCl}_{3}, 360 \mathrm{MHz}\right)$ of $N, N$-diphenyl-p-anisidine (Table 4, entry $10)$.

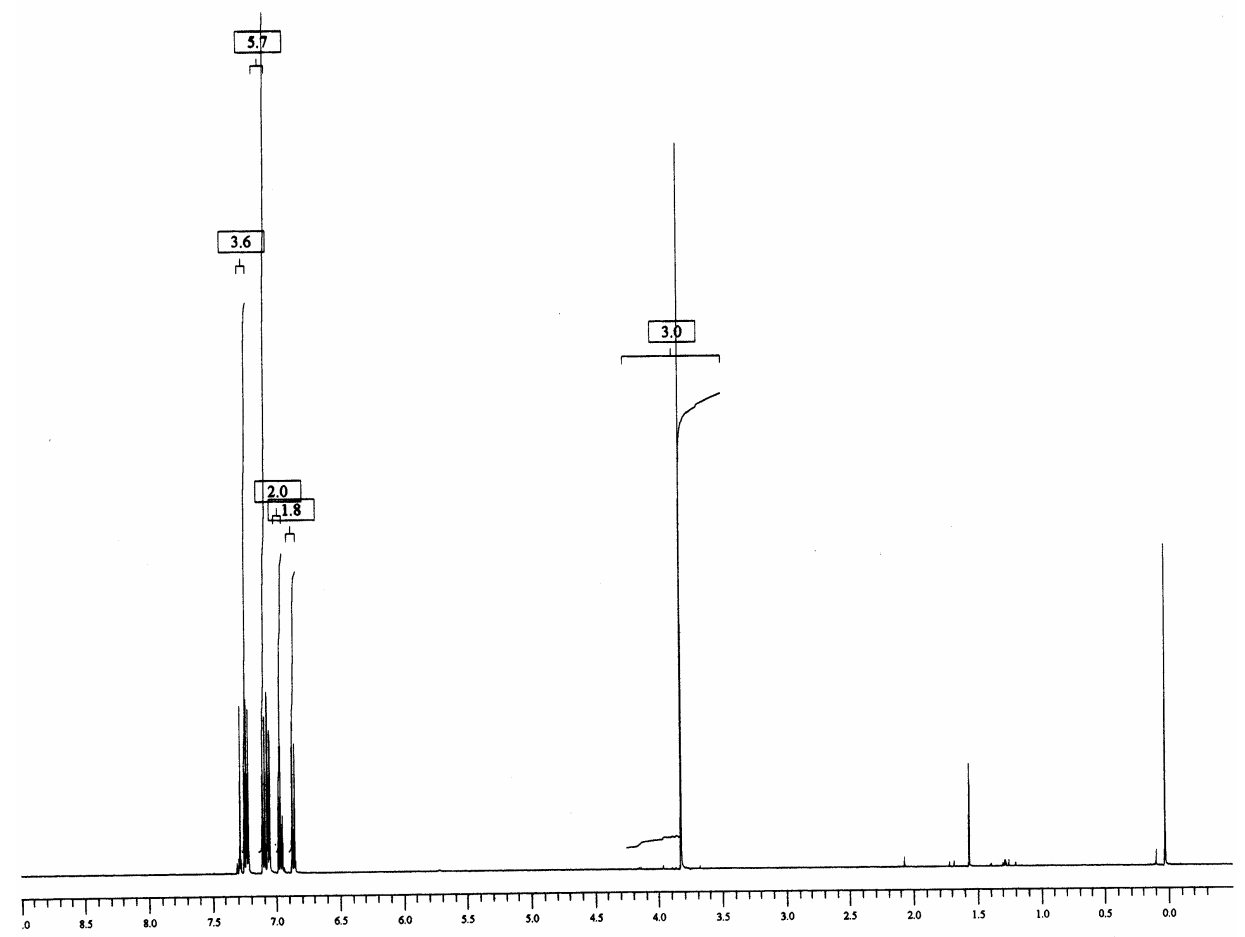

Figure S42. ${ }^{13} \mathrm{C}$ NMR spectrum $\left(\mathrm{CDCl}_{3}, 90.6 \mathrm{MHz}\right)$ of $N, N$-diphenyl-p-anisidine (Table 4, entry $10)$. 

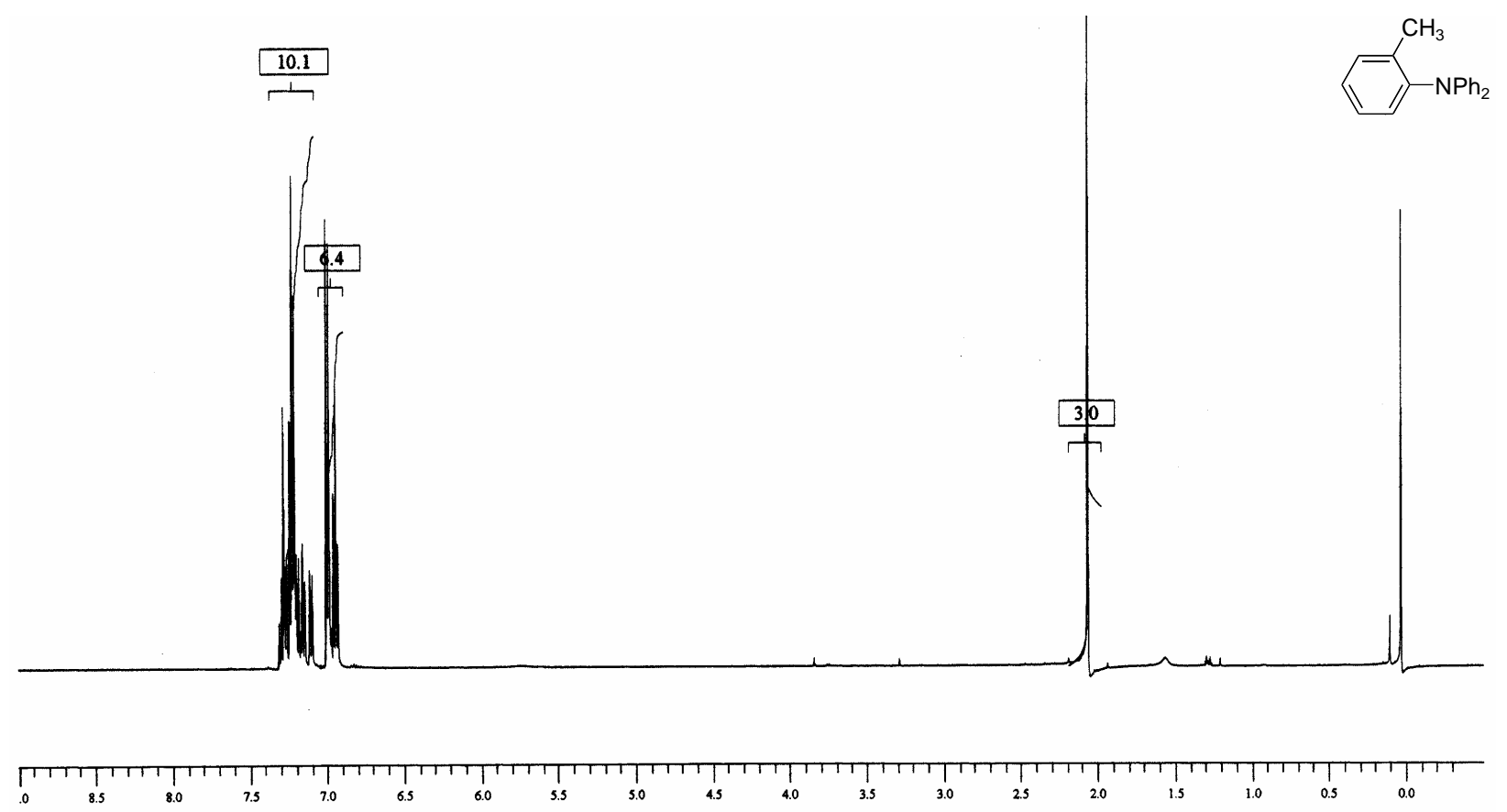

Figure S43. ${ }^{1} \mathrm{H}$ NMR spectrum $\left(\mathrm{CDCl}_{3}, 360 \mathrm{MHz}\right)$ of $N, N$-diphenyl-o-toluidine (Table 4, entry 11).

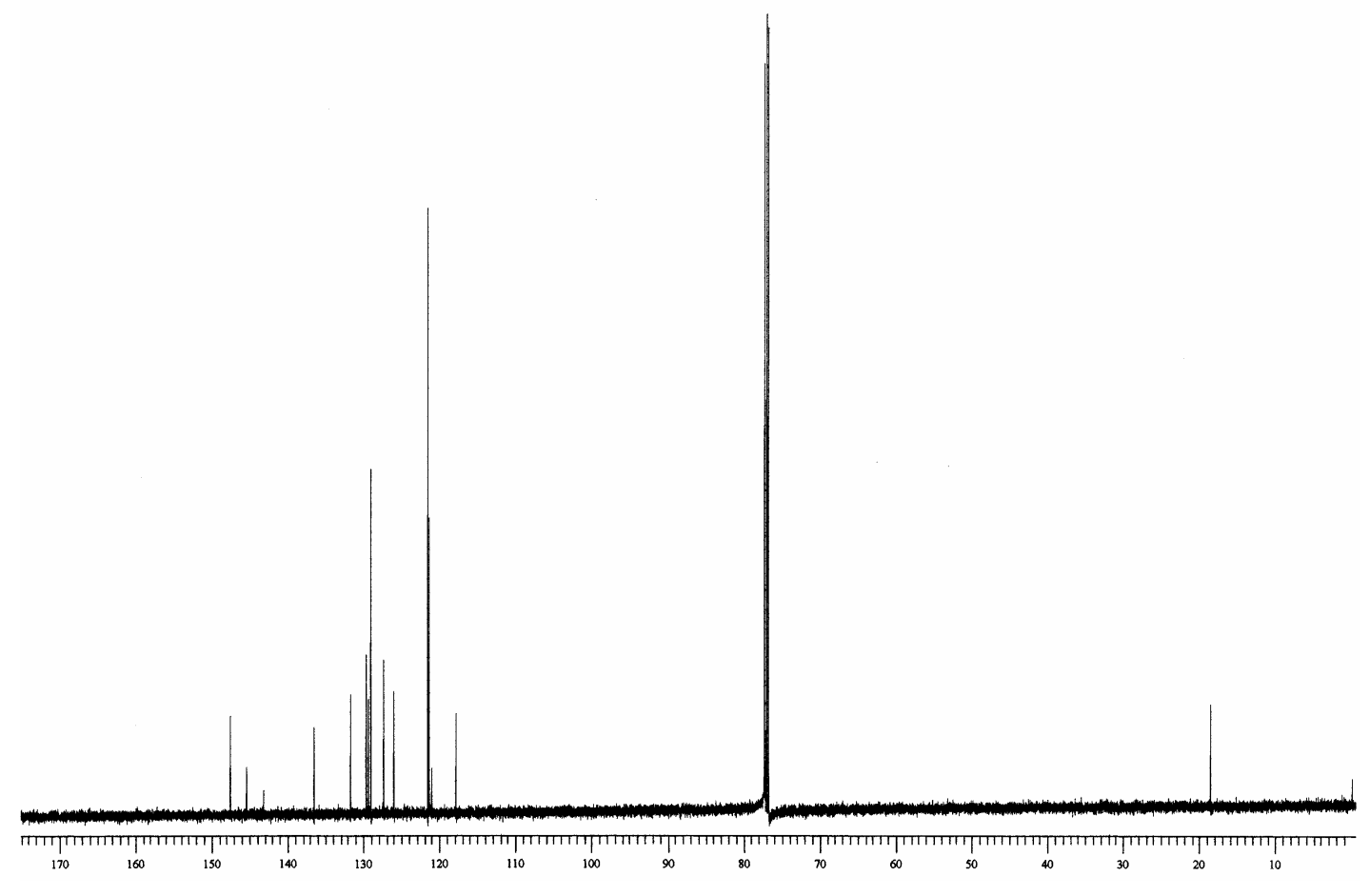

Figure S44. ${ }^{13} \mathrm{C}$ NMR spectrum $\left(\mathrm{CDCl}_{3}, 90.6 \mathrm{MHz}\right)$ of $N, N$-diphenyl-o-toluidine (Table 4, entry 11). 

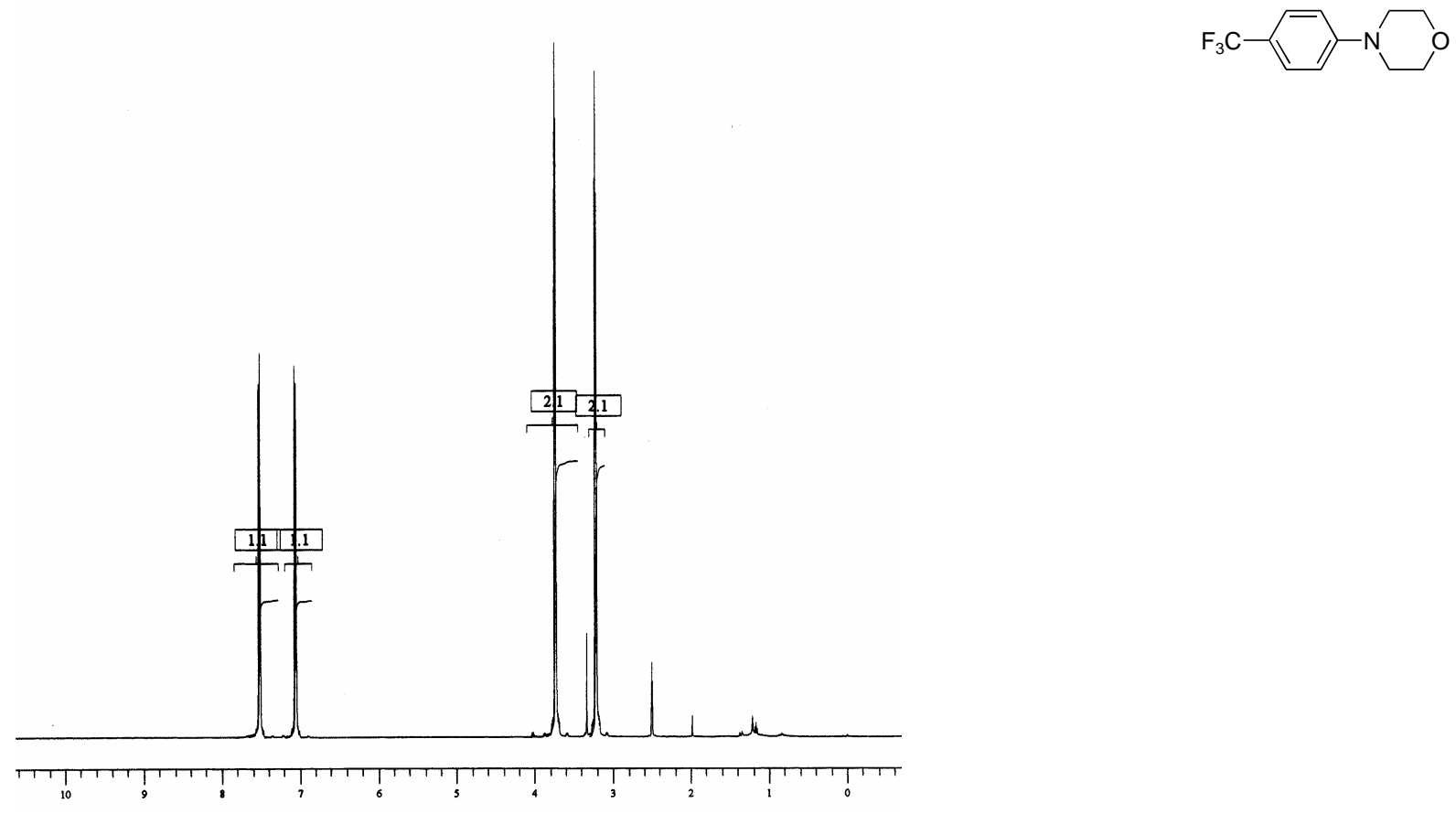

Figure S45. ${ }^{1} \mathrm{H}$ NMR spectrum $\left(\mathrm{CDCl}_{3}, 360 \mathrm{MHz}\right)$ of $\mathrm{N}$-(4-trifluoromethylphenyl)morpholine (Table 5, entry 1).

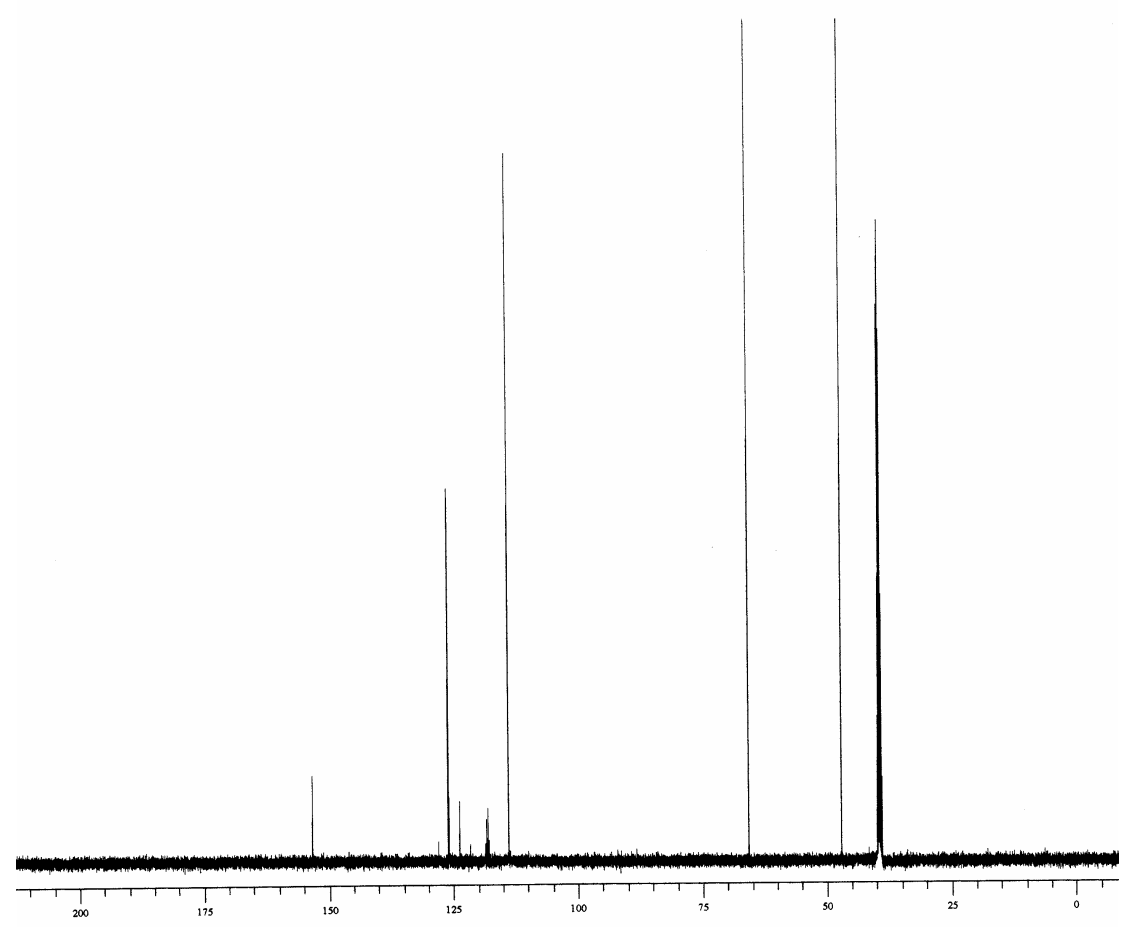

Figure S46. ${ }^{13} \mathrm{C}$ NMR spectrum $\left(\mathrm{CDCl}_{3}, 90.6 \mathrm{MHz}\right)$ of $\mathrm{N}$-(4-trifluoromethylphenyl)morpholine (Table 5, entry 1). 


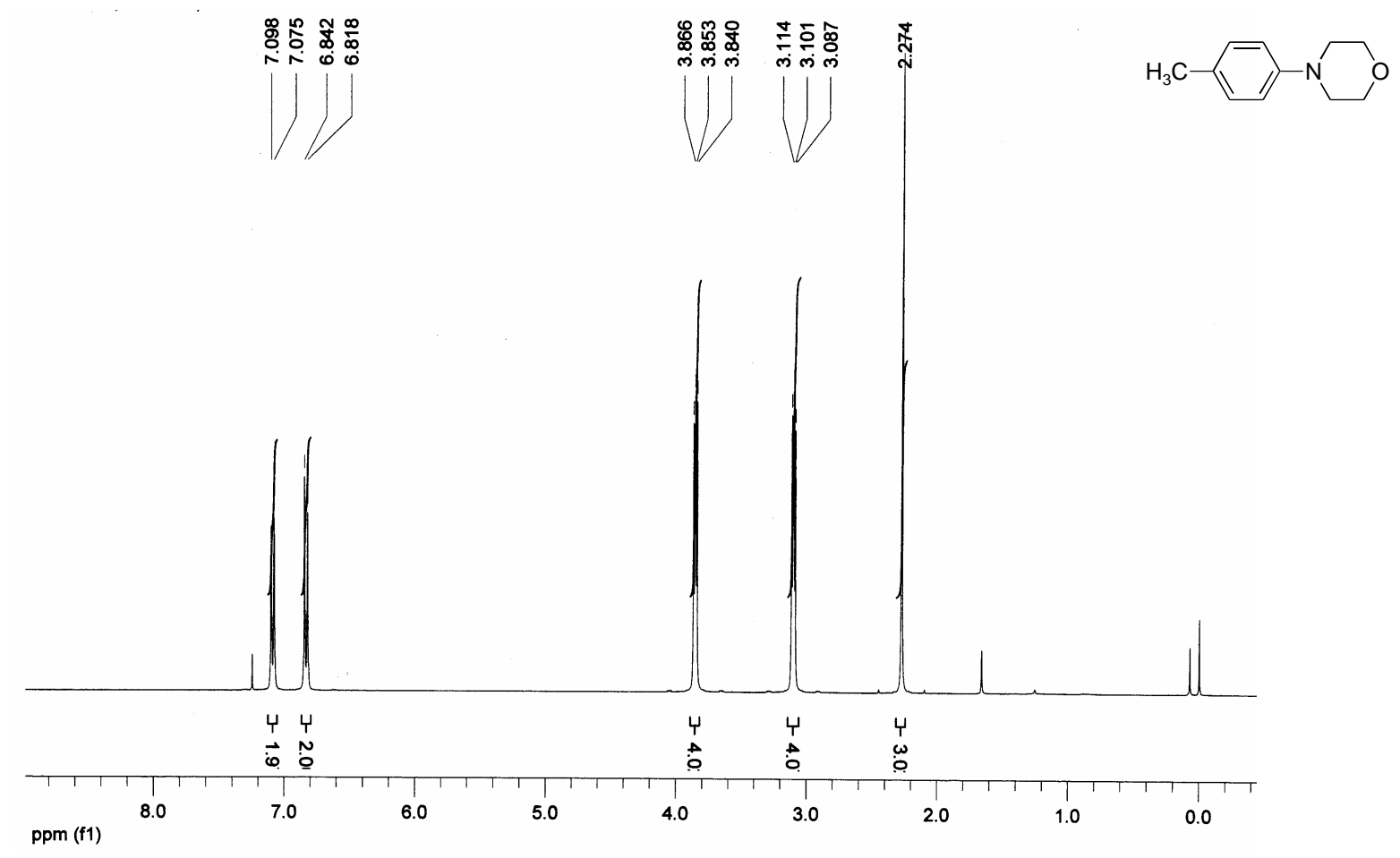

Figure S47. ${ }^{1} \mathrm{H}$ NMR spectrum ( $\mathrm{CDCl}_{3}, 360 \mathrm{MHz}$ ) of $\mathrm{N}$-(4-methylphenyl)morpholine (Table 5, entry 2).

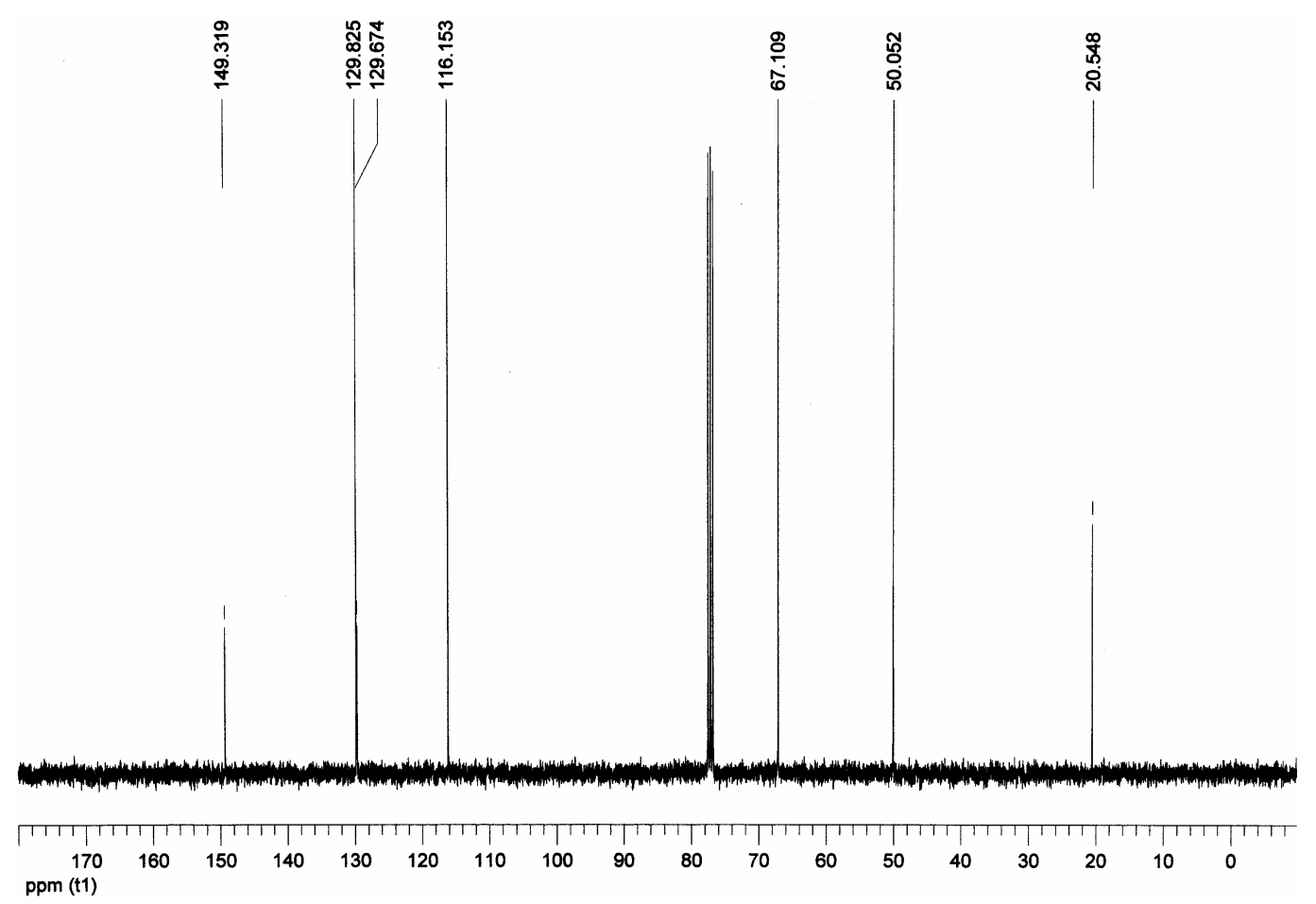

Figure S48. ${ }^{13} \mathrm{C}$ NMR spectrum $\left(\mathrm{CDCl}_{3}, 90.6 \mathrm{MHz}\right)$ of $N$-(4-methylphenyl)morpholine (Table 5, entry 2). 


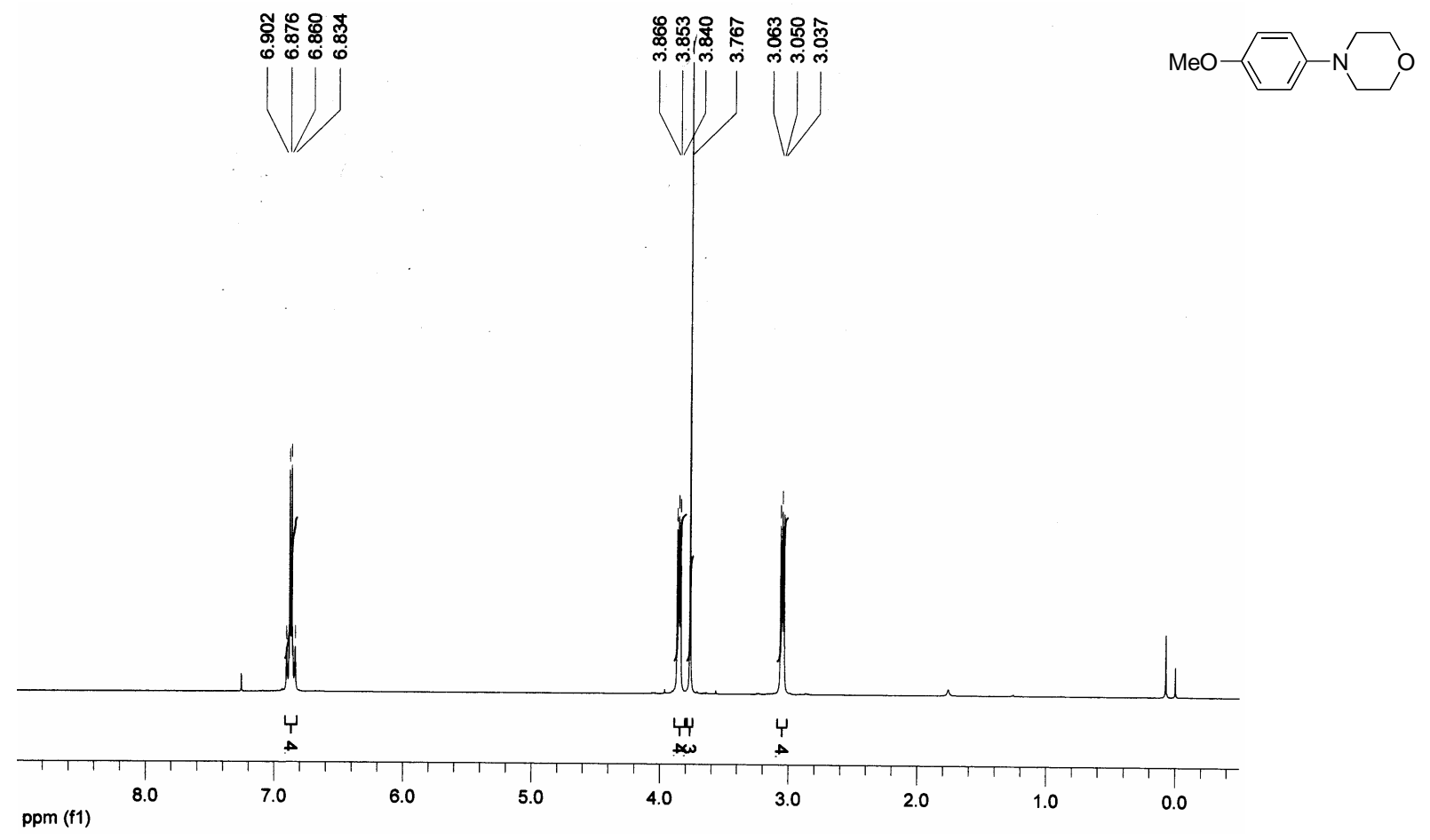

Figure S49. ${ }^{1} \mathrm{H}$ NMR spectrum $\left(\mathrm{CDCl}_{3}, 360 \mathrm{MHz}\right)$ of $N$-(4-methoxyphenyl)morpholine (Table 5, entry 3).

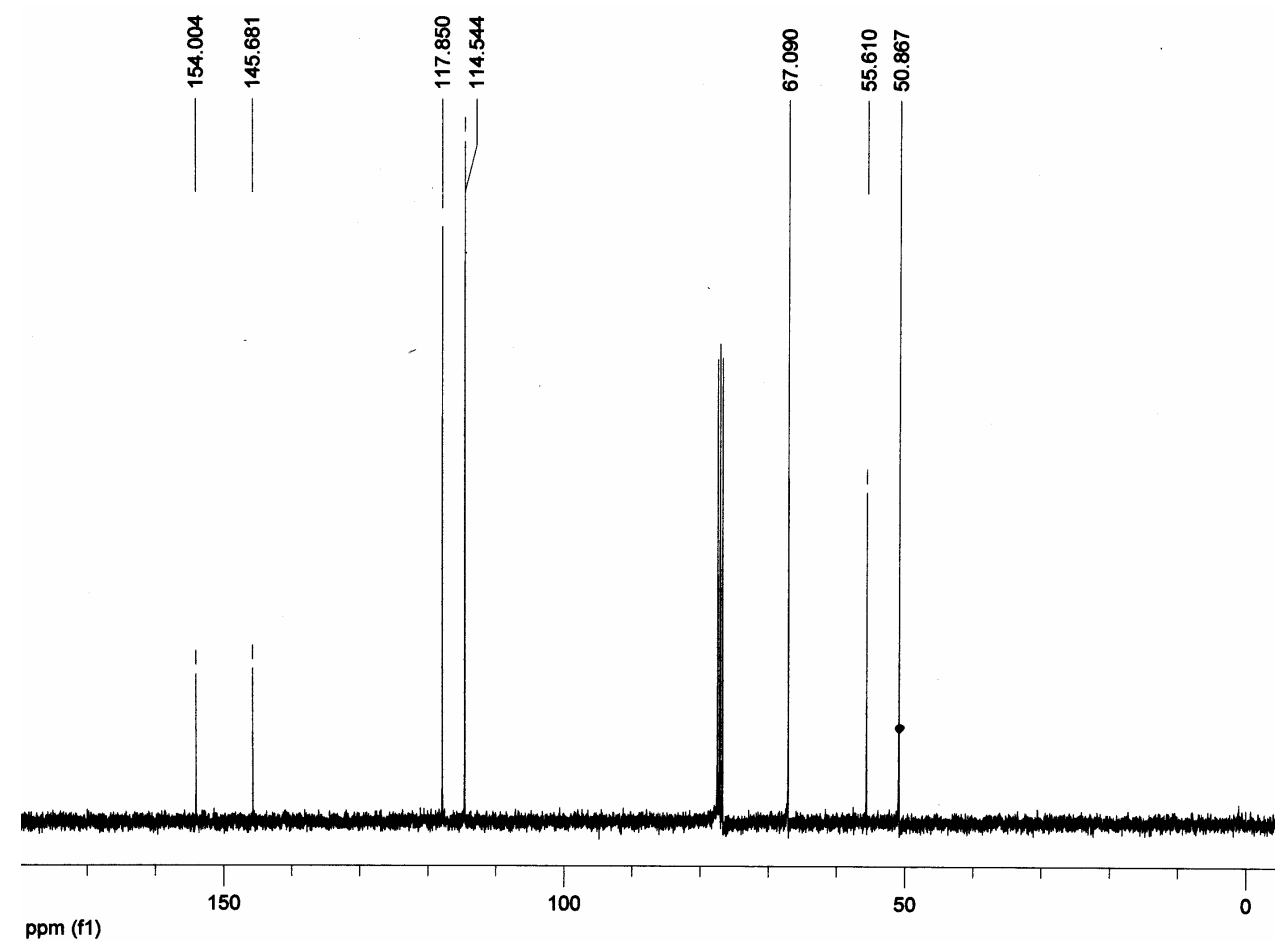

Figure S50. ${ }^{13} \mathrm{C}$ NMR spectrum $\left(\mathrm{CDCl}_{3}, 90.6 \mathrm{MHz}\right)$ of $\mathrm{N}$-(4-methoxyphenyl)morpholine (Table 5, entry 3). 


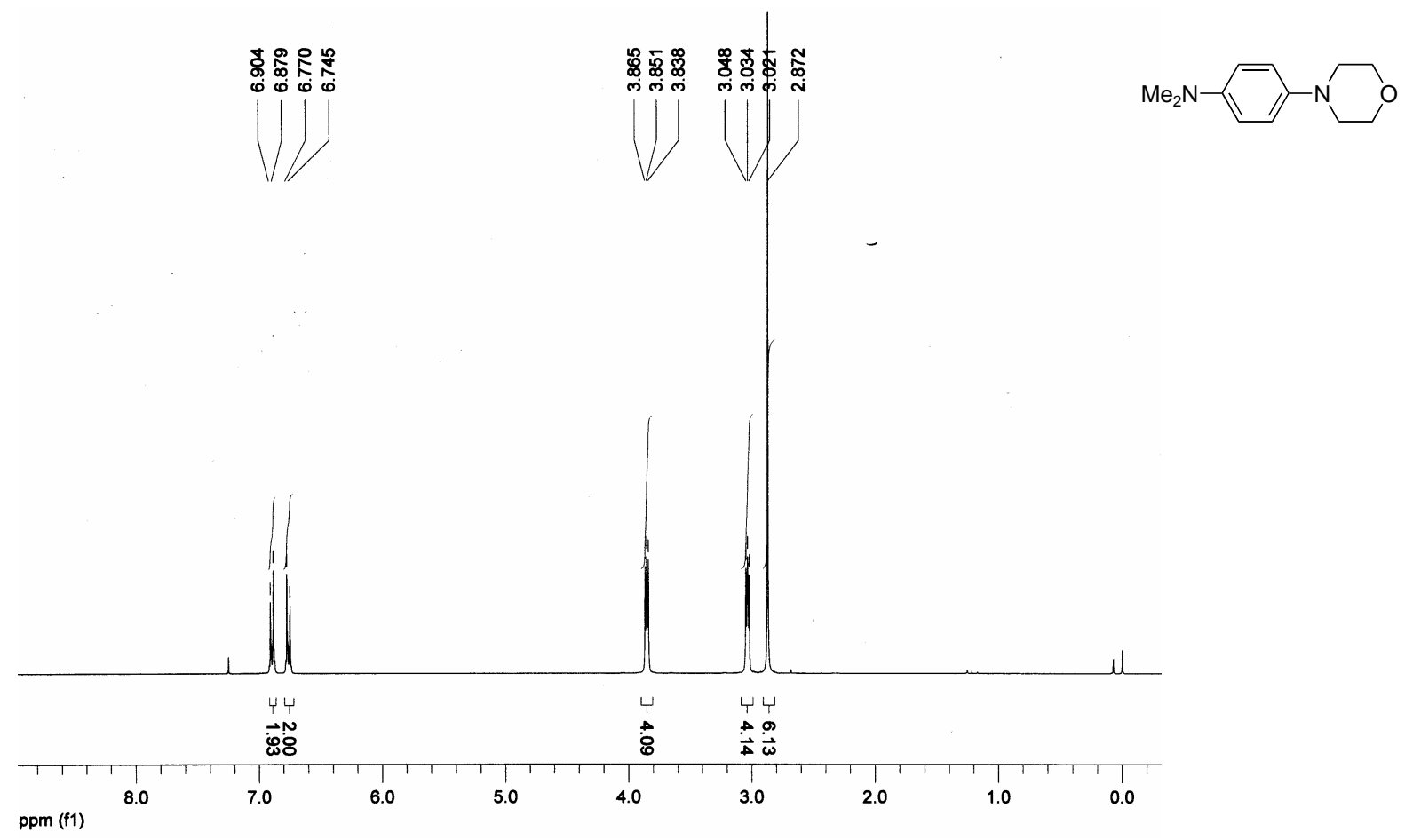

Figure S51. ${ }^{1} \mathrm{H}$ NMR spectrum $\left(\mathrm{CDCl}_{3}, 360 \mathrm{MHz}\right)$ of $N$-(4- $N$ ', $N$ '-dimethylamino)phenyl)morpholine (Table 5, entry 4).

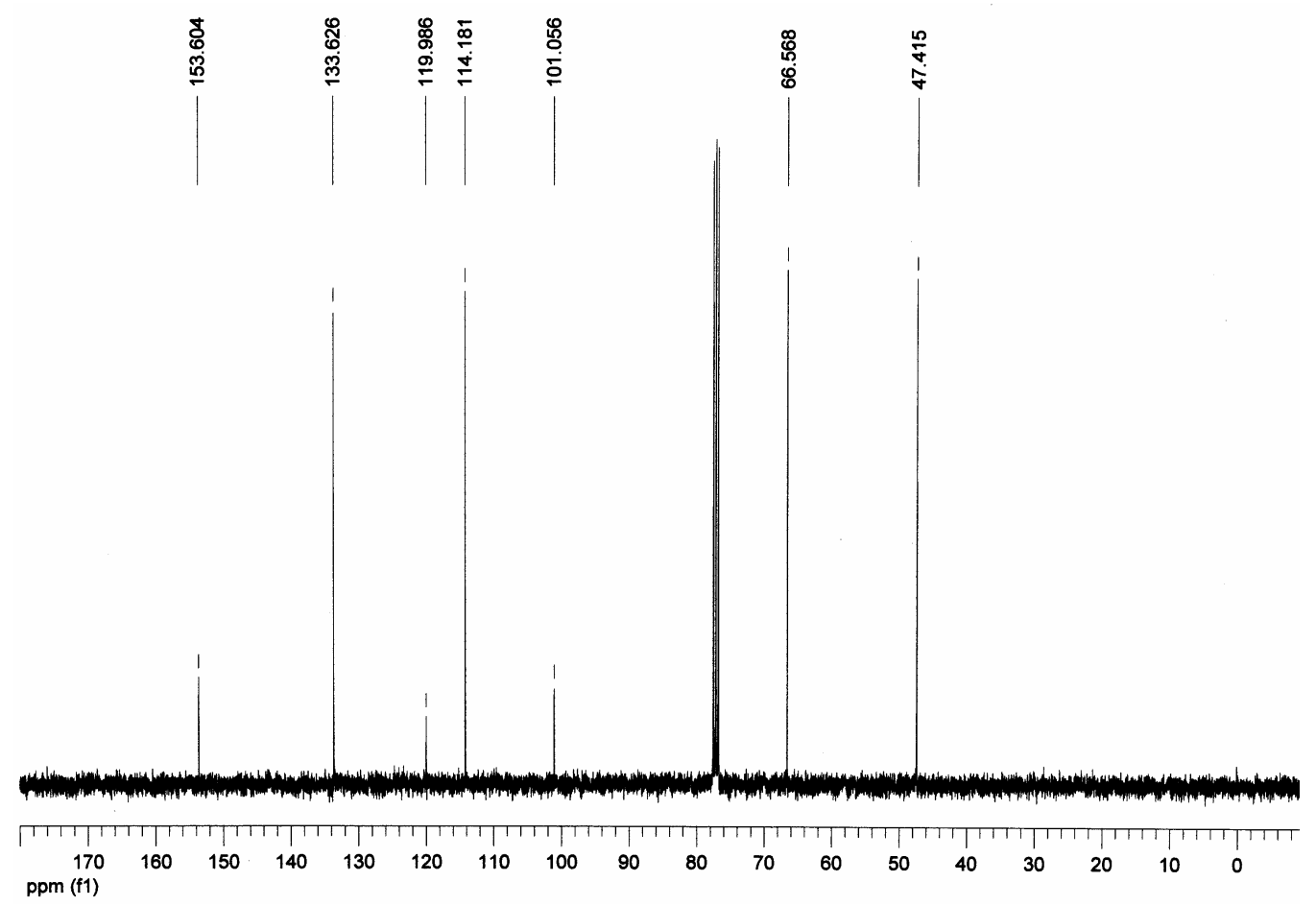

Figure S52. ${ }^{13} \mathrm{C}$ NMR spectrum $\left(\mathrm{CDCl}_{3}, 90.6 \mathrm{MHz}\right)$ of $N$-(4- $N$ ', $N^{\prime}$-dimethylamino)phenyl)morpholine (Table 5, entry 4). 


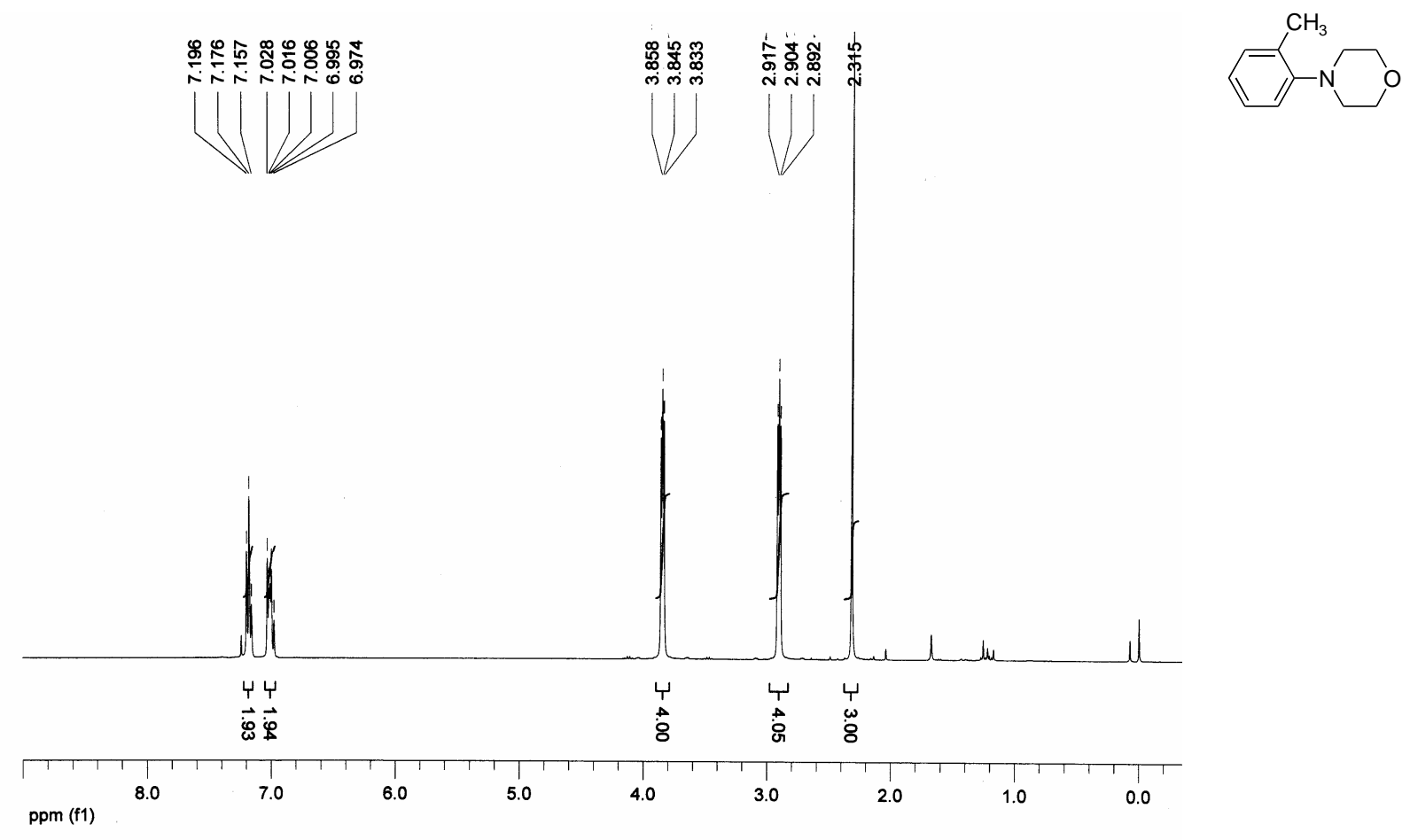

Figure S53. ${ }^{1} \mathrm{H}$ NMR spectrum $\left(\mathrm{CDCl}_{3}, 360 \mathrm{MHz}\right)$ of $\mathrm{N}$-(2-methylphenyl)morpholine (Table 5, entry 5).
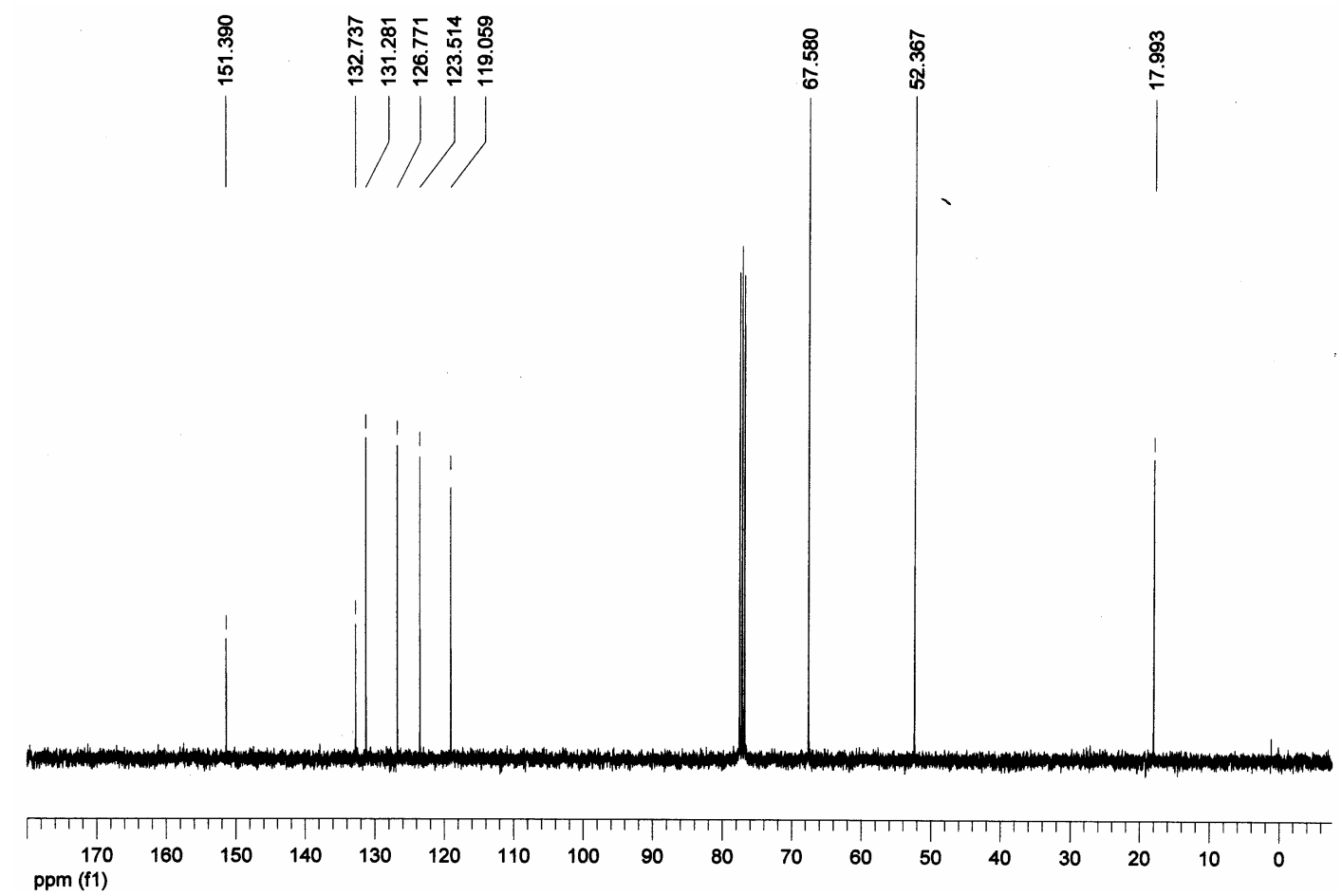

Figure S54. ${ }^{13} \mathrm{C}$ NMR spectrum $\left(\mathrm{CDCl}_{3}, 90.6 \mathrm{MHz}\right)$ of $\mathrm{N}$-(2-methylphenyl)morpholine (Table 5, entry 5). 


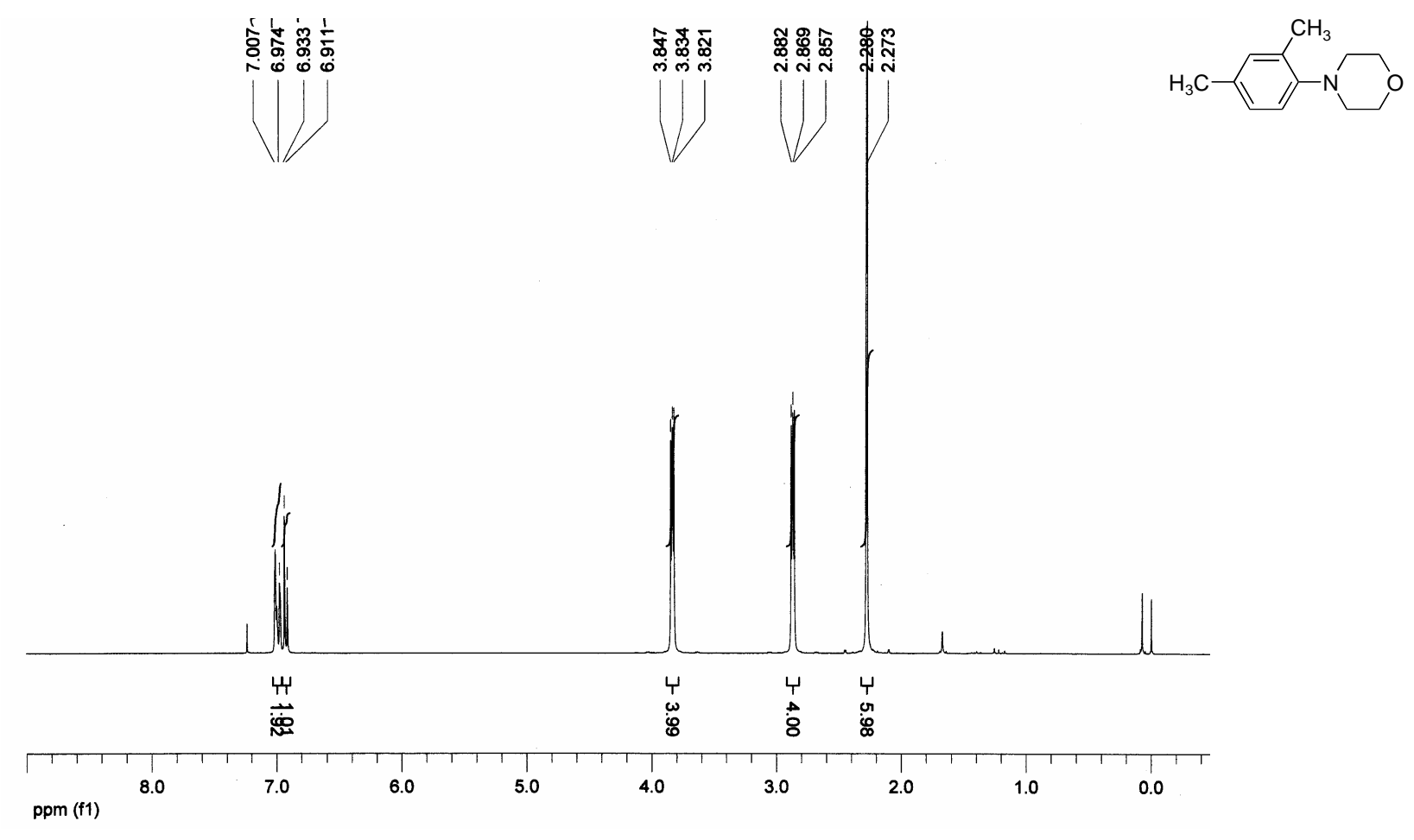

Figure S55. ${ }^{1} \mathrm{H}$ NMR spectrum $\left(\mathrm{CDCl}_{3}, 360 \mathrm{MHz}\right)$ of $N$-(2,4-dimethylphenyl)morpholine (Table 5, entry 6).

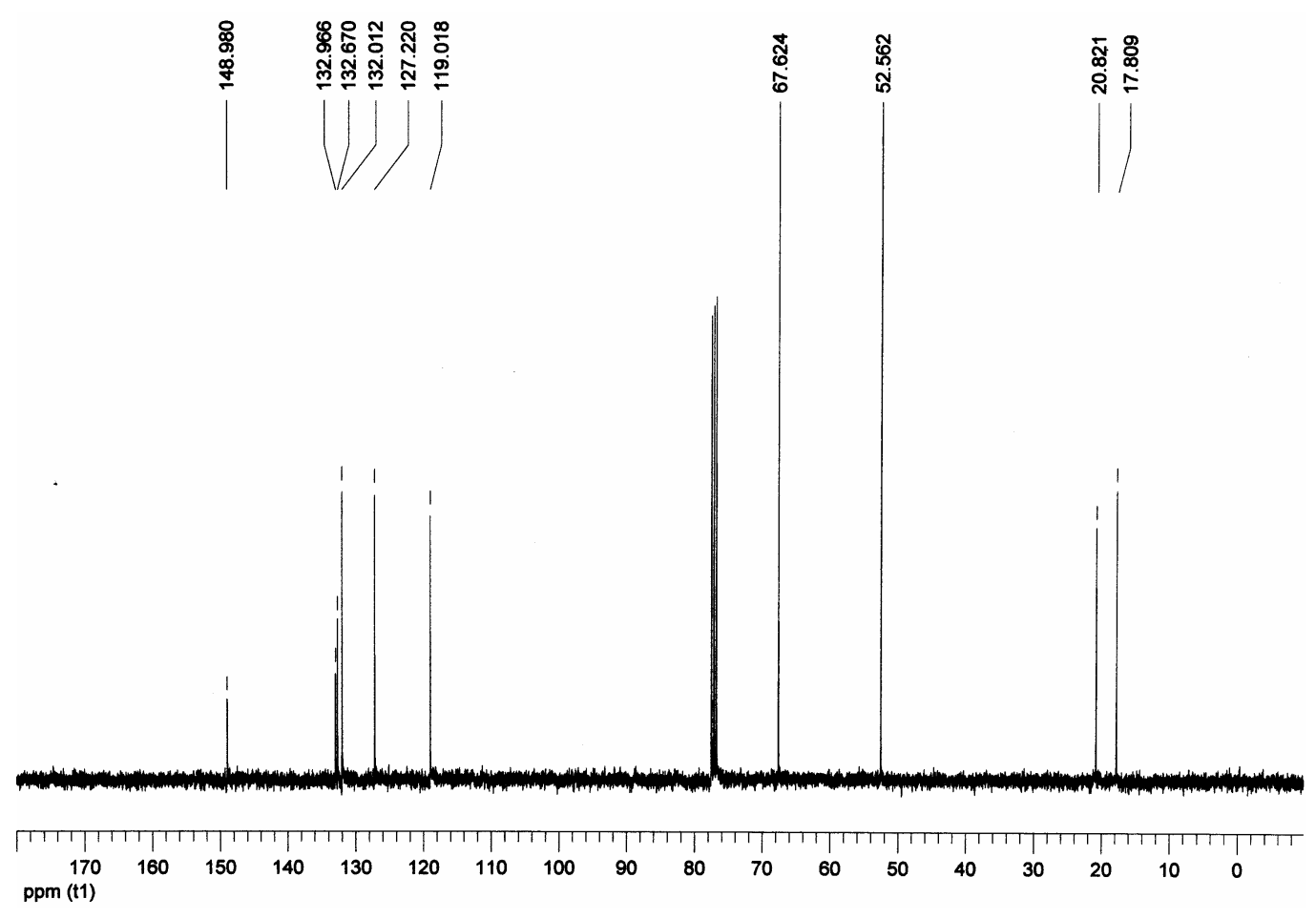

Figure S56. ${ }^{13} \mathrm{C}$ NMR spectrum $\left(\mathrm{CDCl}_{3}, 90.6 \mathrm{MHz}\right)$ of $N$-(2,4-dimethylphenyl)morpholine (Table 5, entry 6). 


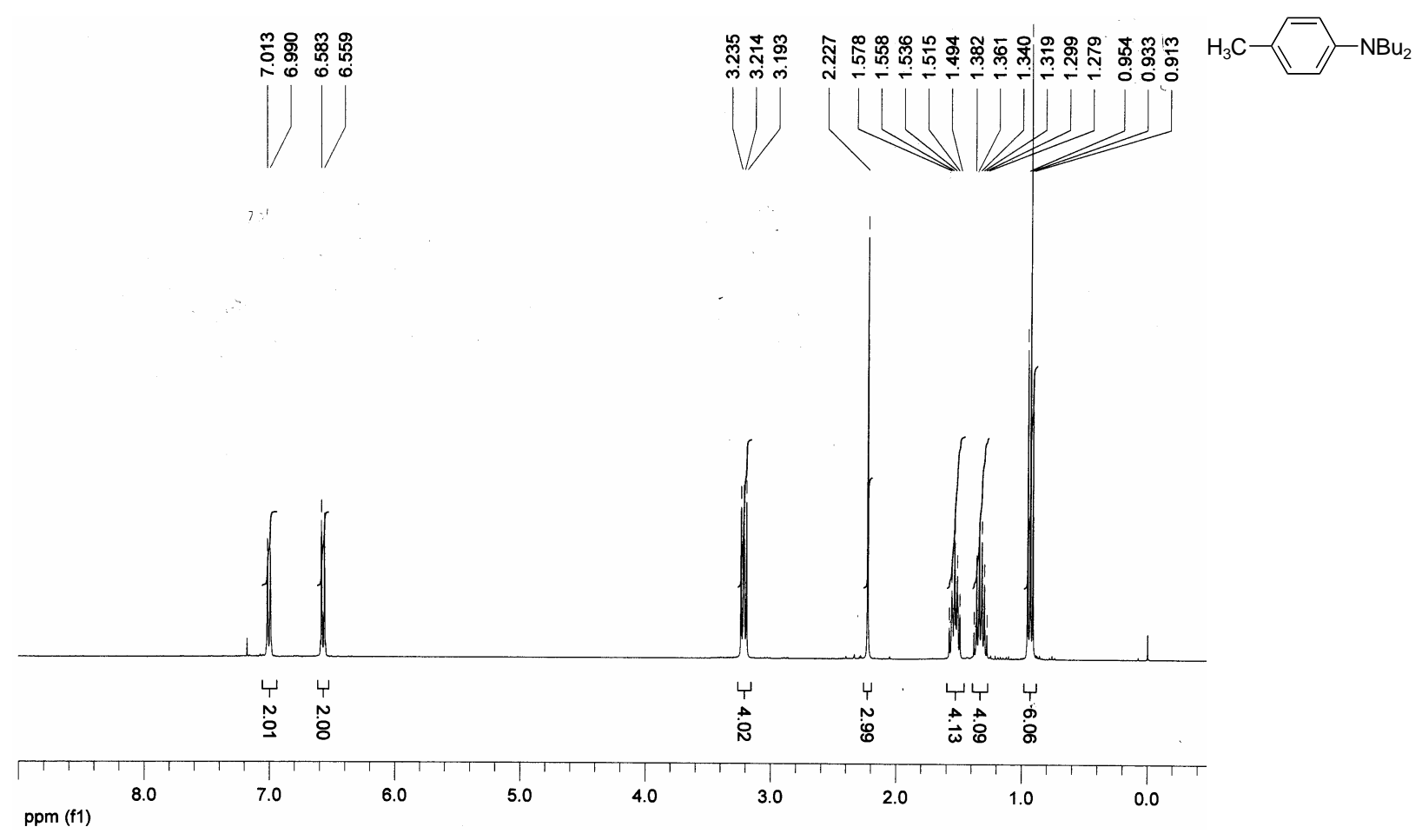

Figure S57. ${ }^{1} \mathrm{H}$ NMR spectrum $\left(\mathrm{CDCl}_{3}, 360 \mathrm{MHz}\right)$ of $N, N$-dibutyl-p-toluidine (Table 5, entry 7).

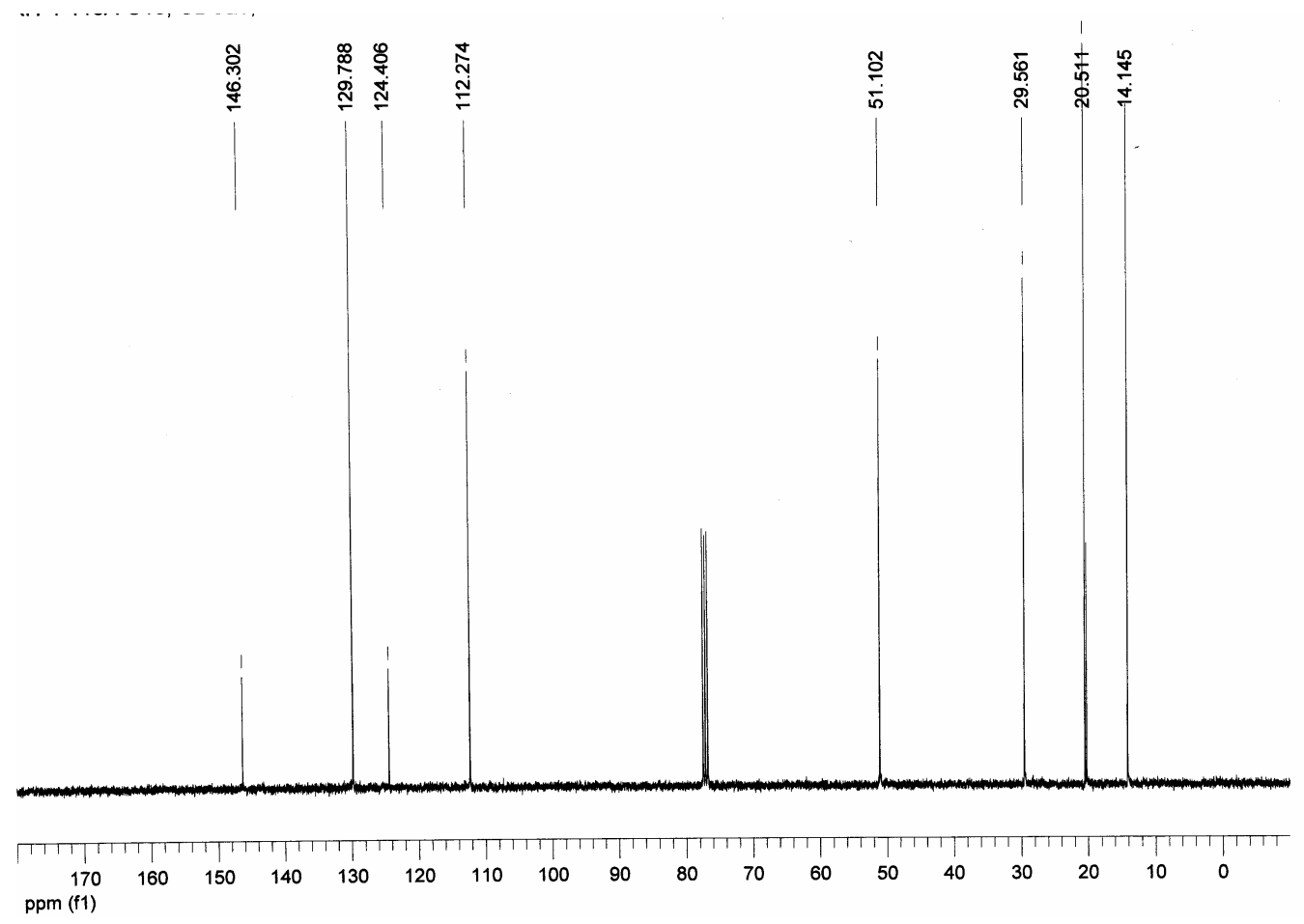

Figure S57. ${ }^{13} \mathrm{C}$ NMR spectrum $\left(\mathrm{CDCl}_{3}, 90.6 \mathrm{MHz}\right)$ of $N, N$-dibutyl-p-toluidine (Table 5, entry 7). 


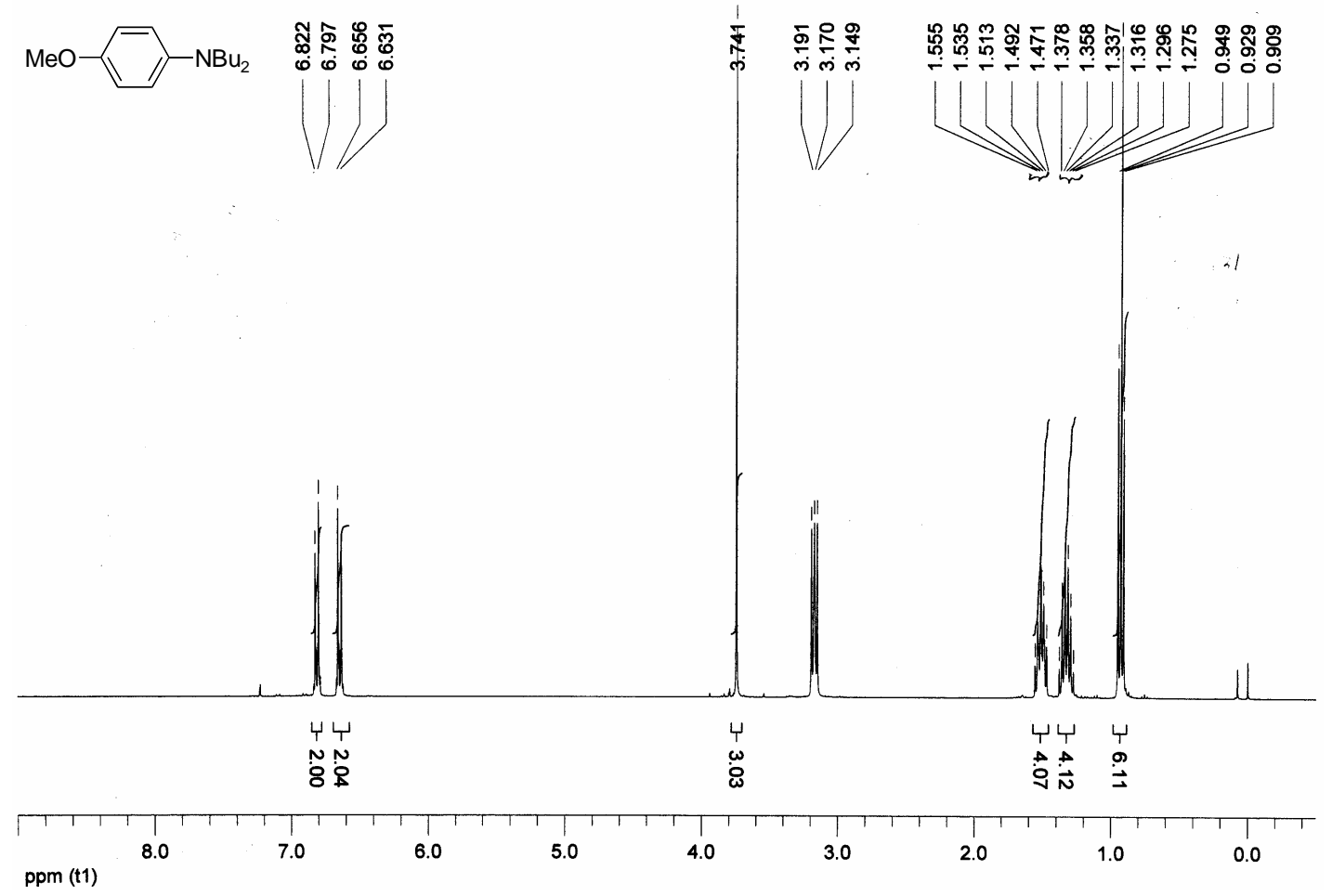

Figure S59. ${ }^{1} \mathrm{H}$ NMR spectrum $\left(\mathrm{CDCl}_{3}, 360 \mathrm{MHz}\right)$ of $N, N$-dibutyl-p-anisidine (Table 5, entry 8).
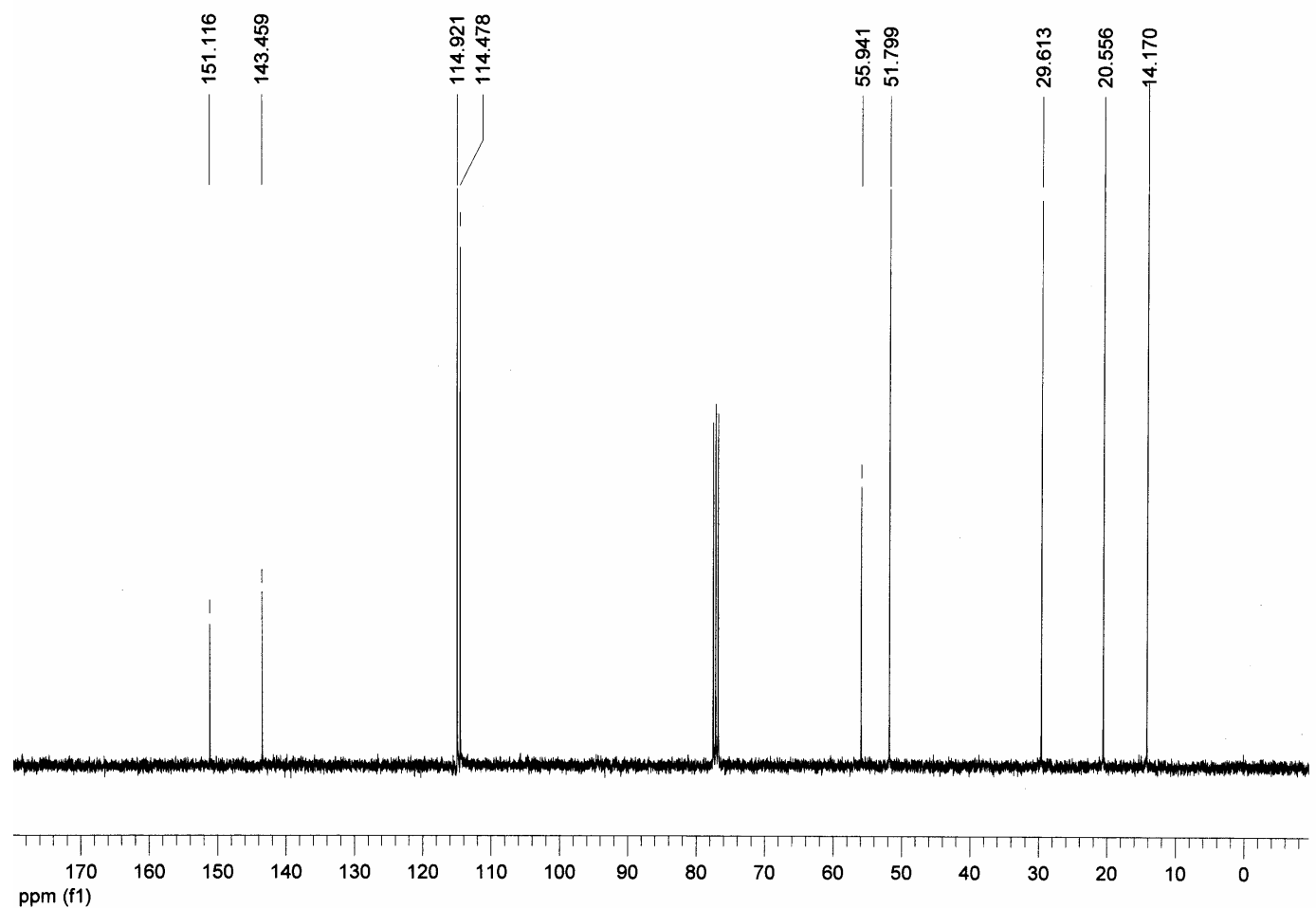

Figure S60. ${ }^{13} \mathrm{C}$ NMR spectrum $\left(\mathrm{CDCl}_{3}, 90.6 \mathrm{MHz}\right)$ of $N, N$-dibutyl-p-anisidine (Table 5, entry 7). 


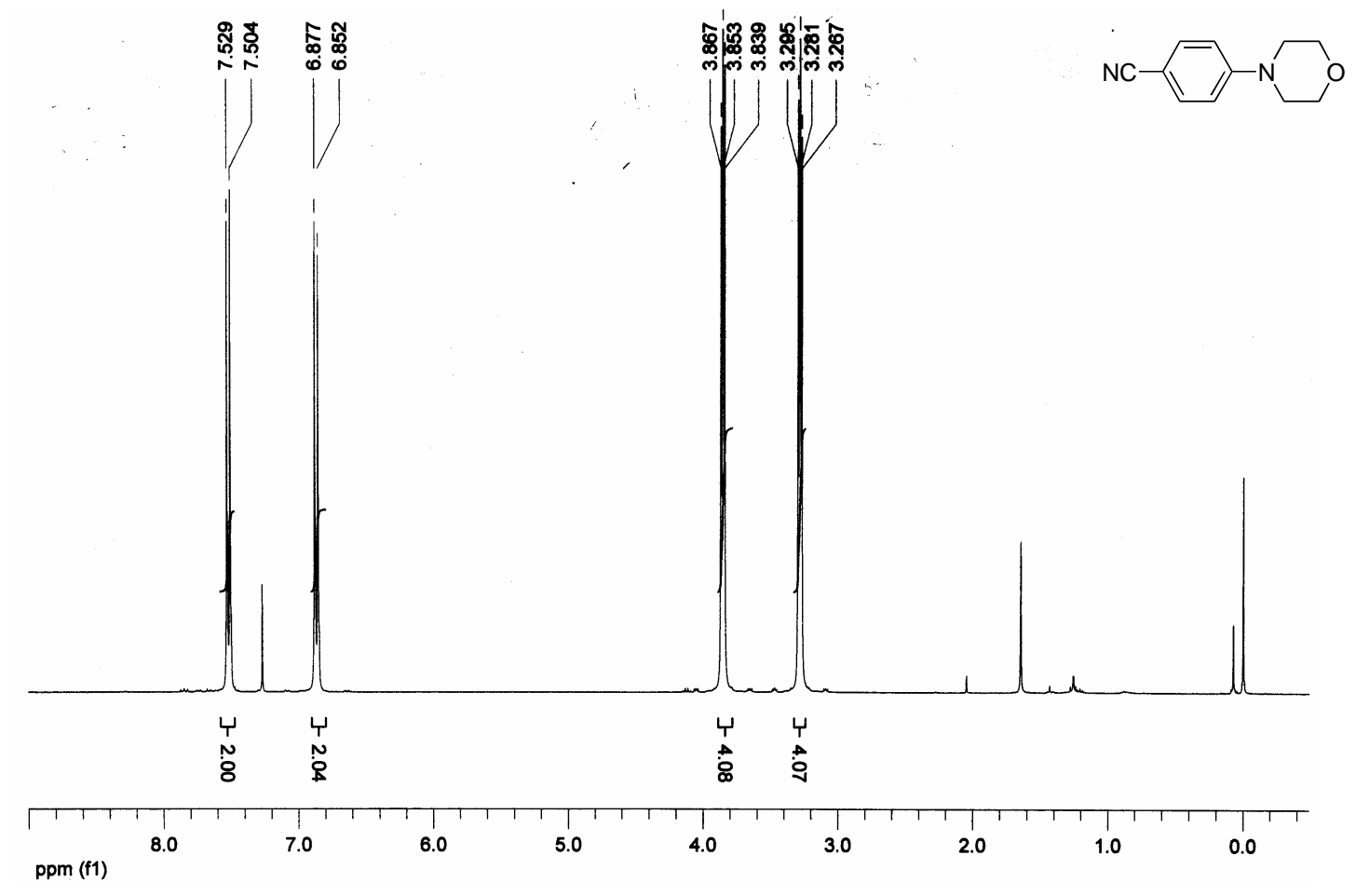

Figure S61. ${ }^{1} \mathrm{H}$ NMR spectrum $\left(\mathrm{CDCl}_{3}, 360 \mathrm{MHz}\right.$ ) of $N$-(4-cyanophenyl)morpholine (Table 6, entry 7).

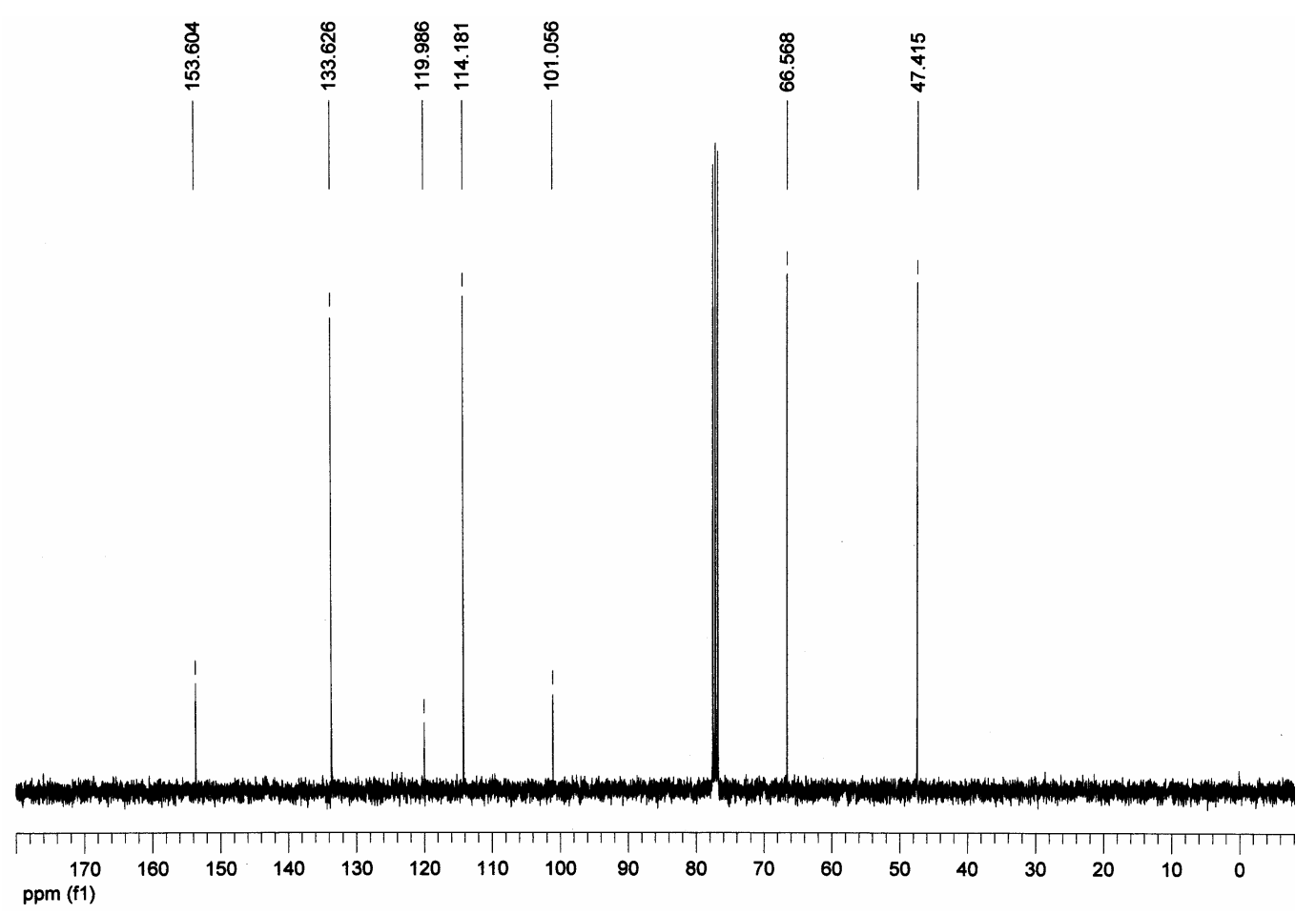

Figure S61. ${ }^{13} \mathrm{C}$ NMR spectrum $\left(\mathrm{CDCl}_{3}, 90.6 \mathrm{MHz}\right)$ of $\mathrm{N}$-(4-cyanophenyl)morpholine (Table 6, entry 7). 